\title{
MEASUREMENTS OF PHYSICAL PARAMETERS OF WHITE DWARFS: A TEST OF THE MASS-RADIUS RELATION
}

\author{
A. Bédard, P. Bergeron, \& G. Fontaine \\ Département de Physique, Université de Montréal, C.P. 6128, Succ. Centre-Ville, \\ Montréal, Québec H3C 3J7, Canada \\ bedard@astro.umontreal.ca, bergeron@astro.umontreal.ca, \\ fontaine@astro.umontreal.ca
}

\begin{abstract}
We present a detailed spectroscopic and photometric analysis of 219 DA and DB white dwarfs for which trigonometric parallax measurements are available. Our aim is to compare the physical parameters derived from the spectroscopic and photometric techniques, and then to test the theoretical mass-radius relation for white dwarfs using these results. The agreement between spectroscopic and photometric parameters is found to be excellent, especially for effective temperatures, showing that our model atmospheres and fitting procedures provide an accurate, internally consistent analysis. Values of surface gravity and solid angle, obtained respectively from spectroscopy and photometry, are combined with parallax measurements in various ways to study the validity of the mass-radius relation from an empirical point of view. After a thorough examination of our results, we find that $73 \%$ and $92 \%$ of the white dwarfs are consistent within 1 and $2 \sigma$ confidence levels, respectively, with the predictions of the mass-radius relation, thus providing strong support to the theory of stellar degeneracy. Our analysis also allows us to identify 15 stars that are better interpreted in terms of unresolved double degenerate binaries. Atmospheric parameters for both components in these binary systems are obtained using a novel approach. We further identify a few white dwarfs that are possibly composed of an iron core rather than a carbon/oxygen core, since they are consistent with Fe-core evolutionary models.
\end{abstract}

Subject headings: stars: fundamental parameters — techniques: photometric techniques: spectroscopic - white dwarfs 


\section{INTRODUCTION}

Our understanding of the nature and the evolution of white dwarf stars begins with the determination of their physical parameters, which include effective temperature, mass, radius, surface gravity, chemical composition, luminosity, cooling age, just to name a few. Some of these parameters are of course related to each other, but the methods used to measure these quantities, directly or indirectly, may differ quite significantly. There are indeed several methods that can be applied to individual stars, or to statistical ensembles, to estimate these parameters. The most commonly used method for stars with strong enough absorption features is the spectroscopic technique, first applied to a large sample of DA white dwarfs by Bergeron et al. (1992a), where the predictions from model atmospheres are compared with high signal-to-noise optical spectra. This technique has been applied in several analyses of relatively bright DA stars (Finlev et al. 1997; Vennes et al. 1997; Liebert et al. 2005; Koester et al. 2009; Gianninas et al. 2011) and in particular to the much fainter DA stars identified in the large spectroscopic SDSS sample (see, e.g., Tremblay et al. 2011a, Kepler et al. 2015), and also to other spectral types as well, including DB stars (Voss et al. 2007; Bergeron et al. 2011; Koester \& Kepler 2015), and DO stars (Reindl et al. 2014). With the spectroscopic technique, the atmospheric parameters measured are the effective temperature, the surface gravity, and the atmospheric composition, because these correspond to the input parameters of model atmosphere calculations. White dwarf masses and other relevant quantities can then be determined through the use of mass-radius relations obtained from evolutionary models appropriate for these stars. The spectroscopic technique can thus be successfully applied with excellent precision to large spectroscopic data (less than $2 \%$ in $T_{\text {eff }}$ and about 0.04 dex in $\log g$ ), given that the spectra have sufficiently high signal-to-noise ratio $(\mathrm{S} / \mathrm{N} \gtrsim 50)$ and that they are properly flux calibrated — which is not always the case.

For cool white dwarfs with weak, or no spectroscopic features - the DC stars, one

must rely on the so-called photometric method (see, e.g., Bergeron et al. 1997) where the spectral energy distribution of a star, built from as many photometric data points as possible, is compared with the predictions of model atmospheres — synthetic photometry in this case. With this method, magnitudes are converted into average fluxes, using appropriate photometric zero points, and compared with model fluxes averaged over the same filter bandpasses. Here both $T_{\text {eff }}$ and the solid angle, $\pi(R / D)^{2}$, are considered free parameters for an assumed atmospheric composition, most commonly pure hydrogen or pure helium. If the distance $D$ to the star is known — from trigonometric parallax measurements for instance its radius $R$ can be obtained directly. The stellar radius can then be converted into mass, and thus $\log g$, using mass-radius relations from evolutionary models. In the absence of distance estimates, one generally assumes an average value of $\log g=8$, from which an estimate of the photometric distance can be derived. Since trigonometric parallaxes are currently 
available for a few hundred white dwarfs (around 350 according to the electronic version of the Catalog of Spectroscopically Identified White Dwarf 1 of McCook \& Sion 1999), the photometric technique can only be applied to a restricted sample of objects. This situation will of course change dramatically when the Gaia mission is completed (see Tremblay et al. 2017 for preliminary results). A hybrid photometric and spectroscopic approach can also be used for fitting DQ and DZ stars, where the effective temperatures and radii are measured from the spectral energy distributions using the photometric method, while the chemical composition is determined from fits to optical spectra, in an iterative fashion (Dufour et al. 2005, 2007).

Gravitational redshift measurements can also be used to measure white dwarf masses of individual stars (Koester 1987; Bergeron et al. 1995a; Reid 1996), or average masses of different spectral types using statistical ensembles (Falcon et al. 2010, 2012). However, for individual measurements, the white dwarf must be part of a common proper motion system in order to determine its systemic velocity, and thus this technique cannot be applied to the majority of field white dwarfs.

While the spectroscopic method is arguably the most precise method to measure the atmospheric parameters of white dwarfs, it is not necessarily the most accurate since theoretical line profiles are very sensitive to the input physics (see Tremblay \& Bergeron 2009 for instance). Also the treatment of convective energy transport at low effective temperatures has been demonstrated to affect significantly the predicted line profiles and overall energy distribution (Bergeron et al. 1992b; Tremblay et al. 2013); for these reasons, most spectroscopic determinations of the atmospheric parameters of DA stars in the past have been restrained to $T_{\text {eff }}>13,000 \mathrm{~K}$, where convective energy transport becomes negligible. While the photometric method is certainly less sensitive to these physical uncertainties, the issues related to photometric calibrations, in particular when combining various photometric systems, will always remain an important problem. More importantly, no matter which method is used, one always needs to rely on mass-radius relations to derive the mass either from $\log g$ values when using the spectroscopic method, or from the measured radius $R$ when using the photometric method (if the distance is known).

Given the new theoretical developments over the last few years regarding model atmosphere calculations (Stark profiles, 3D hydrodynamical calculations, etc. - see references above), and given the large amount of spectroscopic, photometric, and trigonometric parallax data currently available to us, we felt it was appropriate to revisit one more time, and with a more internally consistent approach, the precision and in particular the accu-

\footnotetext{
${ }^{1}$ http://www.astronomy.villanova.edu/WDCatalog/index.html
} 
racy of both the spectroscopic and photometric methods using a well-defined white dwarf sample with measured trigonometric parallaxes. Such comparisons have already been performed using surface gravities (see Figure 7 of Bergeron et al. 1992a), stellar masses (see Figure 19 of Bergeron et al. 2001), absolute magnitudes (see Figure 29 of Gianninas et al. 2011), or even distances (see Figure 22 of Bergeron et al. 2011). In all cases, these comparisons always rely on the mass-radius relation for white dwarfs in order to bring the various physical quantities on the same footing. Specific studies aimed at testing this mass-radius relation for white dwarfs include the analyses of trigonometric parallaxes measured by Hipparcos (Schmidt 1996; Provencal et al. 1998), the analysis by Holberg et al. (2012) based on parallax measurements of 12 DA stars from different sources, while the most recent investigations by Tremblay et al. (2017) and Parsons et al. (2017) rely, respectively, on parallax measurements from the Gaia Data Release 1 and eclipsing binaries. In a few instances, the mass-radius relation can be tested for stars with a measured astrometric mass (see, e.g., Procyon B by Provencal et al. 2002, and Sirius B by Barstow et al. 2015 and Bond et al. 2017), or even from the gravitational deflection of light around a white dwarf - as predicted by Einstein's general theory of relativity - in the particular case of Stein 2051 B (Sahu et al. 2017).

In this paper we present a detailed study of a large sample of photometric, spectroscopic, and trigonometric parallax data aimed at comparing the results of atmospheric parameters obtained from the photometric and spectroscopic techniques, which are then used to test the mass-radius relation for white dwarfs under various assumptions. In Section 2 we describe all the observational data used in our spectroscopic and photometric analyses, the results of which are presented and compared in Section 3. Our test of the mass-radius relation follows in Section 4, where we explain our approach and present a thorough discussion of our findings. We conclude in Section 5 ,

\section{OBSERVATIONAL DATA}

\subsection{Selection of the Sample}

The objects studied in our analysis were selected on the basis of two criteria: the availability of trigonometric parallax measurements, and the ability to constrain the surface gravity from spectroscopy. These two requirements follow from the method we employ to investigate the mass-radius relation, which will be described in detail in Section 4.1. Firstly, trigonometric parallaxes are an essential ingredient for testing the mass-radius relation, as shown in previous studies (Provencal et al. 1998; Holberg et al. 2012; Tremblay et al. 2017), since they provide a way to measure stellar radii directly without invoking the mass-radius 
relation. Secondly, the approach we use to infer white dwarf masses requires spectroscopic determinations of $\log g$, which involves comparing observed and synthetic spectra to measure the atmospheric parameters $\left(T_{\text {eff }}\right.$ and $\log g$ ). Because the spectroscopic method can only be applied successfully to hydrogen and helium line spectra, we restrict our analysis to DA and DB white dwarfs that are hot enough to have sufficiently strong absorption features, that is, DA stars with $T_{\text {eff }} \gtrsim 5500 \mathrm{~K}$ and DB stars with $T_{\text {eff }} \gtrsim 12,000 \mathrm{~K}$.

\subsection{Trigonometric Parallax Measurements}

After a careful search through the literature, we have defined a sample of 206 DA and 13 DB white dwarfs with trigonometric parallax measurements, for a total of 219 objects. The parallax measurements used in our study are drawn from several sources. Our sample comprises 29 white dwarfs for which we adopt the remarkably precise parallax measurements provided by the first data release of the Gaia astrometric mission (Gaia Collaboration 2016). We also rely on new results from the CTIOPI and USNO parallax programs for 46 and 15 objects, respectively (Subasavage et al. 2009, 2017). These new Gaia, CTIOPI and USNO parallaxes have typical uncertainties of only a few percent - even less than $1 \%$ in many cases - making these white dwarfs highly reliable candidates to test the mass-radius relation.

Another good source of parallaxes is the Hipparcos Space Astrometry Mission. Our sample contains 16 objects for which the parallax measurements were taken from the revised version of the Hipparcos catalog (van Leeuwen 2007). Most of these measurements have uncertainties smaller than 10\%, a level of precision that is quite satisfactory for the purpose of our analysis. For 52 stars in our sample, the parallaxes were drawn from the numerous lists published by the USNO in the eighties (Harrington \& Dahn 1980; Dahn et al. 1982, 1988; Harrington et al. 1985). Although this set of measurements is older than the more recent one described above, our level of confidence in the reliability of the USNO data remains high. The uncertainties are typically between 5 and $20 \%$, but can reach nearly $40 \%$ for some objects. For $1639+153$ and 2349-031, we use more recent USNO data taken respectively from Harris et al. (2013) and Dahn et al. (2004). For 57 white dwarfs in our sample, parallax measurements were taken from the Yale Parallax Catalog (van Altena et al. 1995, hereafter YPC); most of the uncertainties range from 5 to $25 \%$, but can reach almost $50 \%$ in some cases. Finally, the parallaxes for two additional objects, 1733-544 and 2351-368, were taken from Ruiz (1996) and Ducourant et al. (2007) with uncertainties of approximately 1 and $10 \%$, respectively.

In the cases where multiple parallax measurements from different sources are available for the same object, we adopt the following order of priority: Gaia, CTIOPI/USNO-new, 
Hipparcos, USNO-old, YPC. This order reflects our level of confidence in the different sources. In particular, we favor the more recent data, which typically have smaller uncertainties than the older measurements. However, we make an exception to this rule: if two parallaxes for the same object show a large discrepancy, we adopt the value that yields the best agreement with the mass-radius relation, because we have reasons to believe that the other measurement is erroneous. The best example is provided by $1314+293$. As discussed by Holberg et al. (2012), the Hipparcos parallax is larger than the YPC parallax by nearly a factor of 2 . If the YPC value is used, the star is consistent with the mass-radius relation, and it is likely that the Hipparcos parallax is simply in error for this object. This reasoning is confirmed by the fact that the more recent Gaia measurement (used in the present study) agrees with the YPC measurement.

The distribution of parallax uncertainties for the 219 white dwarfs in our sample is displayed in Figure 1. Also shown is the same distribution but for the 158 objects that were kept in our refined sample used to investigate the mass-radius relation, after our critical analysis of all the available trigonometric parallax, spectroscopic, and photometric data, described in Section [3. Finally, we show in Figure 2 the number of white dwarfs in both samples as a function of distance.

\subsection{Spectroscopic Data}

Among the 206 DA white dwarfs included in our sample, four are DAZ stars since their spectra show traces of metals, and five are members of DA+M dwarf systems. Also, seven out of the 13 DB white dwarfs have small amounts of hydrogen and are thus DBA stars.

Five objects in our sample are confirmed to be weakly magnetic: $0257+080$ (Koester et al. 2009), 1953-011 Koester et al. 1998, 2009), 2047+372 (Landstreet et al. 2016), 2105-820 (Landstreet et al. 2012), and 2359-434 (Landstreet et al. 2012), but we have intentionally excluded all strongly magnetic white dwarfs, which cannot be modeled with sufficient accuracy in the context of our theoretical framework.

High signal-to-noise spectroscopic observations were gathered for all white dwarfs in our sample, particularly in the blue portion of the spectrum $(\lambda \sim 3700-5200 \AA)$ to cover the hydrogen Balmer line series and most of the important neutral helium lines, but we also secured spectra at $\mathrm{H} \alpha$ to constrain the hydrogen abundance in DB stars. Most of the spectroscopic data for DA and DB stars were retrieved from our previous white dwarf studies (Bergeron et al. 1992a; Liebert et al. 2005; Subasavage et al. 2007, 2008, 2009; Bergeron et al. 2011; Gianninas et al. 2011; Giammichele et al. 2012; Limoges et al. 2015); the spectral resolution in all cases ranges from $2 \AA$ to $9 \AA$ FWHM. We also make use of 


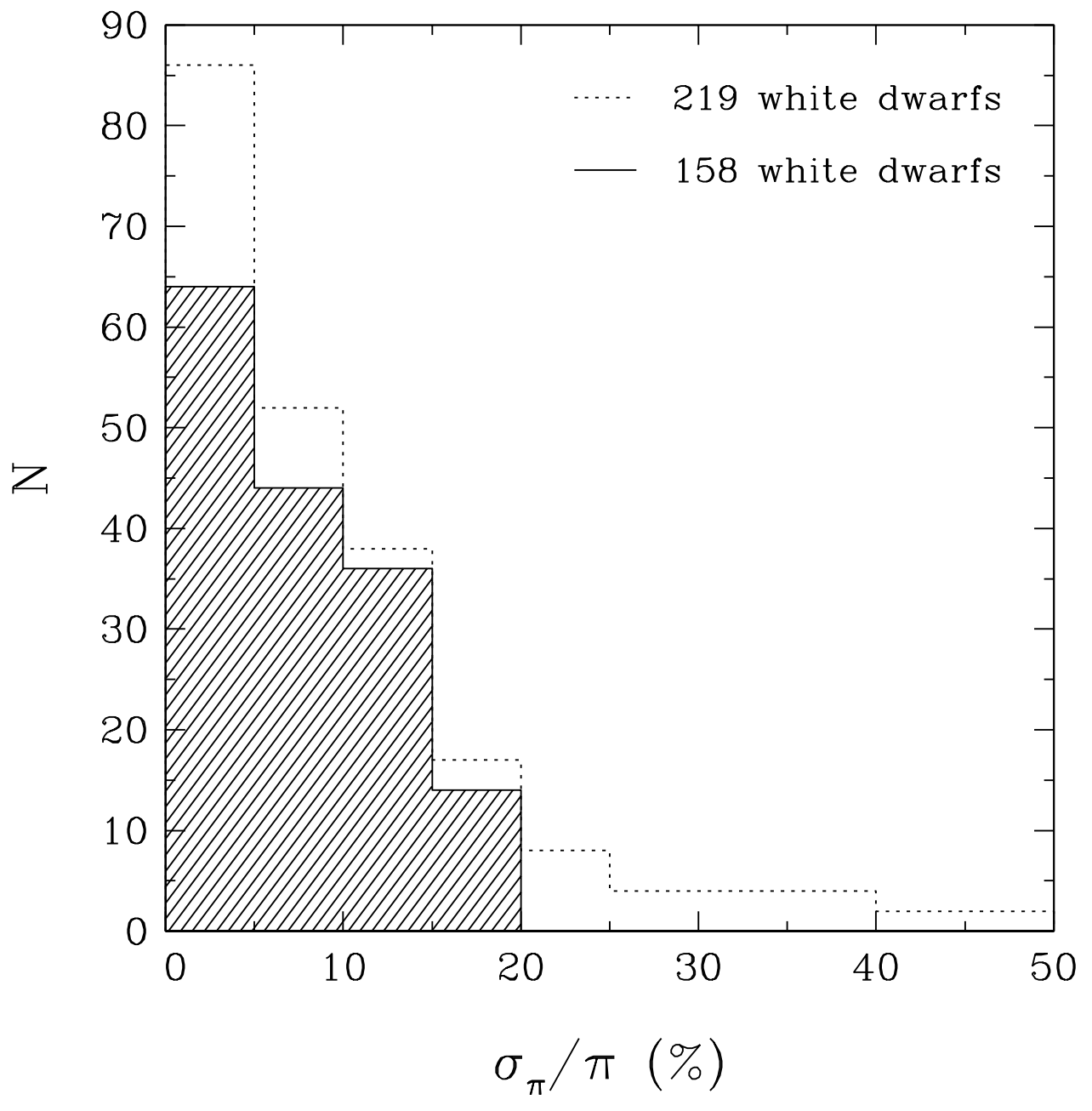

Fig. 1.- Distribution of parallax uncertainties for the 219 white dwarfs in our sample. The hatched histogram shows the distribution for the 158 objects with reliable trigonometric parallax and spectroscopic data used in our test of the mass-radius relation (see Section 4). 


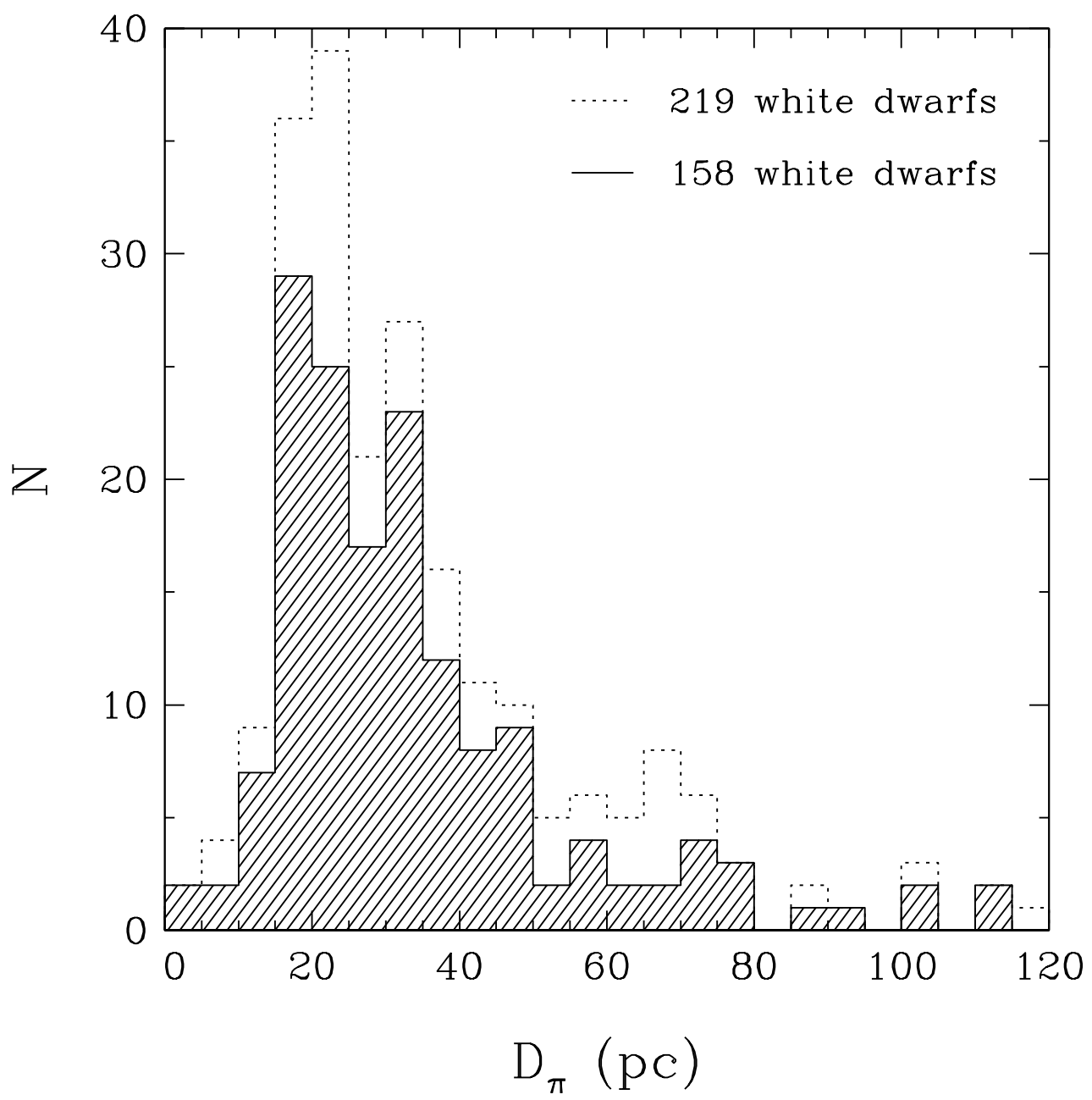

Fig. 2.- Distribution of distances obtained from the parallax measurements for the 219 white dwarfs in our sample. The hatched histogram shows the distribution for the 158 objects with reliable trigonometric parallax and spectroscopic data used in our test of the mass-radius relation (see Section 4). 
high-resolution (<1 $\mathrm{A}$ FWHM) observations from the ESO SN Ia Progenitor Survey (SPY; Napiwotzki et al. 2003).

\subsection{Photometric Data}

For each object in our sample, we searched the literature for several sets of photometric data, namely Johnson BVRI, SDSS ugriz, and Strömgren uvby photometry in the optical, as well as Johnson $J H K$ and 2MASS $J H K_{s}$ photometry in the infrared. Our purpose was to constrain as best as possible the energy distribution for each star by using simultaneously the available magnitudes in all bandpasses during the fitting procedure.

The bulk of our Johnson $B V R I$ and $J H K$ photometry was taken from the studies of cool white dwarfs by Bergeron et al. (1997, 2001, hereafter BRL97 and BLR01, respectively), and from the study of ZZ Ceti stars by Bergeron et al. (2009). For many objects, $V R I$ magnitudes were taken from the studies of white dwarfs in the solar neighborhood by Subasavage et al. (2007, 2008, 2009, 2017). We also collected photometric measurements from additional sources, including $B V R I$ from Landolt (1992, 2009, 2013), Holberg et al. (2008), and Koen et al. (2010), as well as $B V$ from the USNO parallax lists mentioned above and from Kidder et al. (1991). The ugriz photometry was drawn directly from the SDSS database, while magnitudes in the Strömgren system were taken from several sources, namely Graham (1972), Wegner (1979, 1983), Lacombe \& Fontaine (1981), Koester \& Weidemann (1982), and Fontaine et al. (1985). We also make use of photometric data in the infrared from the Two Micron All-Sky Survey (2MASS; Cutri et al.|2003). For one object, 0030+444, we rely on multichannel $U B G V R I$ photometry from Greenstein (1976).

\section{ATMOSPHERIC PARAMETERS DETERMINATION}

\subsection{Spectroscopic Analysis}

The spectroscopic analysis of the DA stars in our sample relies on the so-called spectroscopic technique first described in Bergeron et al. (1992a) and improved by Bergeron et al. (1995b) and Liebert et al. (2005). Briefly, the observed Balmer line profiles are normalized to a continuum set to unity, either by using pseudo-Gaussian profiles or model spectra, and then compared with model spectra convolved with the appropriate Gaussian instrumental profile. A $\chi^{2}$ value is defined by comparing observed and model spectra of all normalized Balmer lines from $\mathrm{H} \beta$ to $\mathrm{H} 8$, which is then minimized using the nonlinear least-squares method of Levenberg-Marquardt (Press et al. 1986) to obtain the best fitting 
parameters, $T_{\text {eff }}$ and $\log g$. The model atmospheres and synthetic spectra we use for the DA stars are similar to those described in Tremblay \& Bergeron (2009) and references therein, which are pure hydrogen models where convective energy transport is treated within the ML2 $/ \alpha=0.7$ version of the mixing-length theory, and where non-LTE effects are taken into account above $T_{\text {eff }}=30,000 \mathrm{~K}$. We also apply the $3 \mathrm{D}$ corrections in both $T_{\text {eff }}$ and $\log g$ given in Tremblay et al. (2013) to take into account hydrodynamical effects. For the DAZ stars in our sample, we rely on the fitting technique and model atmospheres described in Gianninas et al. (2011), which include the opacity from Ca II H \& K known to contaminate the $\mathrm{H} \epsilon$ line.

Formal uncertainties in each fitted parameter can be obtained from the covariance matrix of the fitting algorithm; these internal errors can become very small if the signal-to-noise ratio of the spectrum is very high, however, and these have never proven very useful in the context of the spectroscopic method. Instead, multiple observations of the same star on different nights, or even different observing runs, provide a much more realistic estimate of the external error, which basically gives a measure of the repeatability of the atmospheric parameter measurements. By using this approach, Liebert et al. (2005) estimated that for DA stars, uncertainties of $1.4 \%$ in $T_{\text {eff }}$ and 0.042 dex in $\log g$ could be achieved 2 . It is important to stress that these uncertainties do not only reflect the precision of the spectroscopic method itself, but also strongly depend on the quality of the observations.

Because the hydrogen lines reach their maximum strength near $T_{\text {eff }} \sim 13,000 \mathrm{~K}$, two temperature solutions exist when using the spectroscopic method, one on each side of the peak of the equivalent widths. In the absence of photometric information, one needs to make an educated guess at which of the cool or hot solution is the most appropriate (see, e.g., Gianninas et al. 2011). This ambiguity is not present in our analysis since we also derive photometric temperatures for each star in our sample, which can be used to discriminate between the cool and the hot solutions.

Sample fits for DA stars of particular interest (see Section 4) are displayed in Figure 3. Since the accuracy of the spectroscopic technique depends on well-defined absorption line profiles, the determination of surface gravity for stars with either a low effective temperature or a noisy spectrum can become somewhat unreliable. For DA stars in particular, the presence of the high Balmer lines in the optical spectrum is critical since these lines are the most sensitive to variations of $\log g$. Thus, some objects initially included in our sample were flagged as uncertain after a careful examination of the spectroscopic results. All in all,

\footnotetext{
${ }^{2}$ The uncertainties given in Section 2.4 of Liebert et al., $1.2 \%$ in $T_{\text {eff }}$ and 0.038 dex in $\log g$, are erroneous, and those provided here are the correct values, as shown in Figure 8 of Liebert et al.
} 


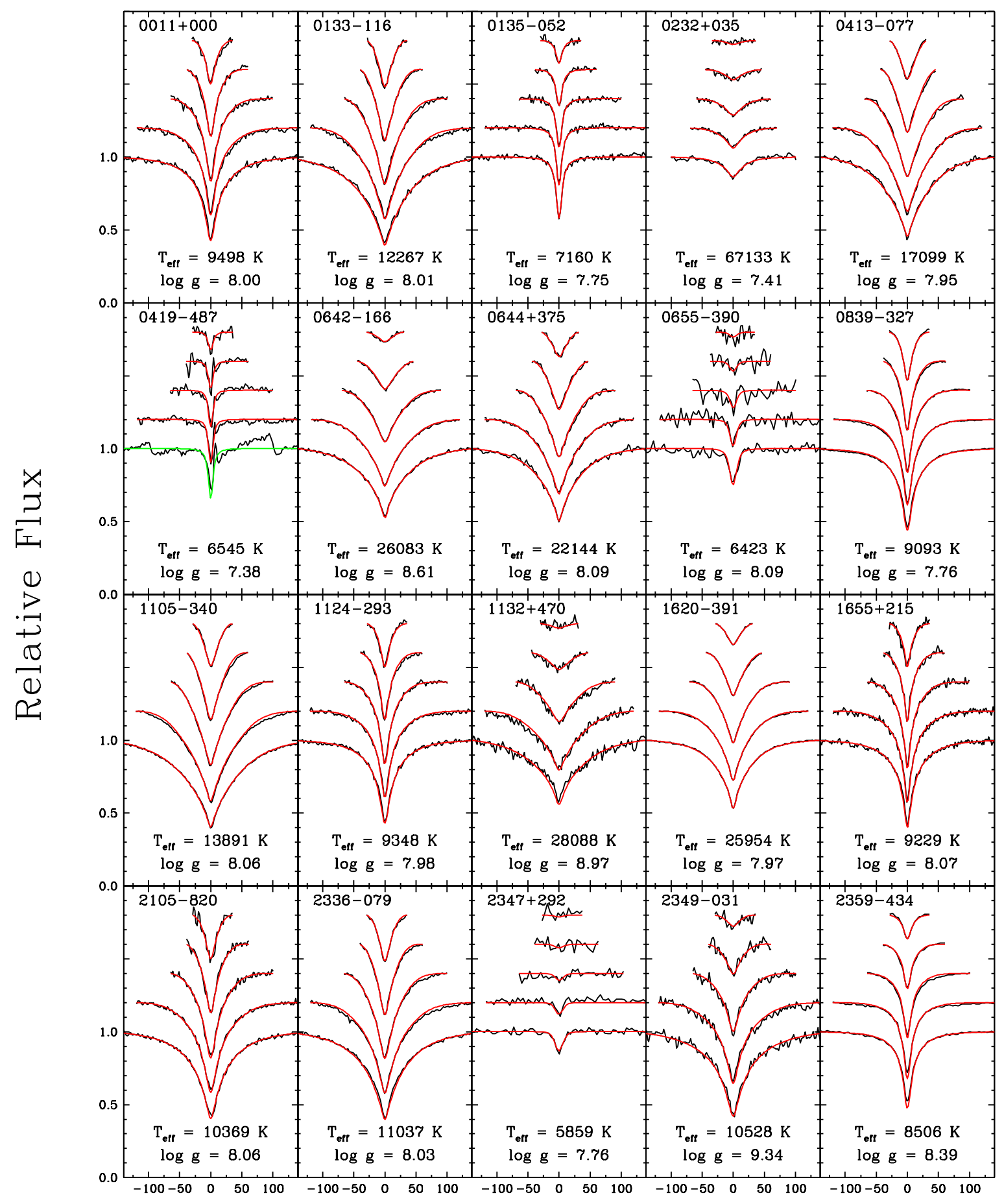

\section{$\Delta \lambda(\AA)$}

Fig. 3.- Fits to the optical spectra of some DA stars in our sample. The lines range from $\mathrm{H} \beta$ (bottom) to H8 (top), each offset vertically by a factor of 0.2 . Theoretical line profiles shown in green are not used in the fitting procedure. The 3D corrections from Tremblay et al. (2013) have also been applied for both $T_{\text {eff }}$ and $\log g$. 
35 DA stars in our sample were too cool (32 objects) or had spectra of too poor quality (3 objects) to allow a significant $\log g$ determination. Note that the $\log g$ values for these objects are not necessarily inaccurate, but have to be considered uncertain in the context of our test of the mass-radius relation.

For binary systems composed of a white dwarf and an $\mathrm{M}$ dwarf companion, the optical spectrum of the former is usually contaminated by the latter, especially at $\mathrm{H} \beta$. This is the case for one of the five WD + M dwarf systems in our sample, 0419-487, shown in Figure 3. To circumvent this problem, we simply exclude the $\mathrm{H} \beta$ line from the fitting procedure for this object, and the atmospheric parameters are derived from the uncontaminated absorption lines.

Our sample also contains 13 DB white dwarfs, seven of which are DBA stars. The atmospheric parameters for these white dwarfs, including also the hydrogen abundance $N(\mathrm{H}) / N(\mathrm{He})$ - or upper limits when $\mathrm{H} \alpha$ is absent, are obtained using the same model atmospheres, synthetic spectra, and fitting procedure as those described at length in Bergeron et al. (2011, see also Bergeron et al. 2015), and the details will not be repeated here. It is worth noting, however, that we exclude from our reliable subsample two DBA stars, one whose line profiles are too weak to be fitted with the spectroscopic method (1917-077), and one that is too cool to yield a reliable $\log g$ measurement $(2147+280)$, a problem most likely related to inaccuracies in the treatment of line broadening at low effective temperatures (see Bergeron et al. 2011 and references therein).

\subsection{Photometric Analysis}

The photometric technique used in our analysis is described at length in BRL97 (see

also Giammichele et al. 2012 for further improvements). Briefly, the magnitudes $m$ in all photometric systems - described in Section 2.4 - are converted into average fluxes, $f_{\lambda}^{m}$, using the zero points given in Holberg \& Bergeron (2006). These are compared with the model Eddington fluxes, $H_{\lambda}^{m}$, properly averaged over the appropriate filter bandpass. These two average fluxes are related by the equation

$$
f_{\lambda}^{m}=4 \pi(R / D)^{2} H_{\lambda}^{m}
$$

where $R / D$ defines the ratio of the radius of the star to its distance from Earth. A $\chi^{2}$ value is defined in terms of the difference between observed and model fluxes over all bandpasses, properly weighted by the photometric uncertainties, which is then minimized using the method of Levenberg-Marquardt mentioned previously. In the above equation, the Ed- 
dington fluxes $H_{\lambda}^{m}$ depend on $T_{\text {eff }}, \log g$, and chemical composition. In our fitting procedure, both $T_{\text {eff }}$ and the solid angle $\pi(R / D)^{2}$ are considered free parameters, for an assumed chemical composition, and the uncertainties of each fitted parameter are obtained directly from the covariance matrix of the fitting algorithm. Since trigonometric parallaxes are available for all objects in our sample, the radius $R$ can be obtained directly from the solid angle and the distance $D$. The value of $\log g$ used to calculate the model fluxes $H_{\lambda}^{m}$ can be determined from the radius combined with mass-radius relations, or alternatively, can be set to the spectroscopic value. In standard photometric analyses (e.g., BRL97, BLR01), the former approach is used, while the latter approach is preferred when studying the validity of the mass-radius relation for white dwarfs (see Section 4.1). We note, however, that the particular choice of $\log g$ for estimating the model fluxes does not affect the measured photometric temperatures significantly, as demonstrated by Genest-Beaulieu \& Bergeron (2014, see their Figure 7).

Because the radius measured from the photometric method depends strongly on the precision and accuracy of the trigonometric parallax measurements, BLR01 excluded from their analysis all parallaxes with uncertainties larger than $30 \%$. Since we want to eventually test the mass-radius relation for white dwarfs in our analysis described below, we adopt a more severe constraint and exclude all objects with uncertainties larger than $20 \%$, that is, 24 objects out of our initial sample of 219 white dwarfs with measured parallaxes.

Particular attention must be paid to white dwarfs in DA+M dwarf systems, since the $\mathrm{M}$ dwarf companion contaminates the energy distribution in the infrared, and even in the optical in some cases. To prevent this contamination, the infrared measurements for $0232+035$, 0419-487, 0628-020, and 1314+293 were not included in our photometric analysis. The $R$ and $I$ magnitudes were also omitted in the cases of $0232+035$ and $0419-487$. For $0518+333$, we use the uncontaminated $B V$ and $J H K$ measurements from Farihi (2009).

Finally, a special treatment was required to analyze the energy distribution of Sirius B (0642-166). Due to the overwhelming luminosity of Sirius A, the only reliable photometric measurement available is the $V$ magnitude, so the photometric technique cannot be applied since magnitudes in several bandpasses are needed to constrain the effective temperature. However, the spectrum we have for Sirius B is that obtained by the Hubble Space Telescope (Barstow et al. 2005), for which the observed flux is absolutely calibrated. Hence, the energy distribution can be analyzed by comparing absolute model fluxes to the observed spectrum at all wavelength. Thus, in the framework of this spectrophotometric approach, the spectroscopic data are used in place of the lacking photometric measurements.

Photometric fits for all 219 objects in our complete sample are displayed in Section 4 when we discuss the mass-radius relation for white dwarfs. 


\subsection{Comparison of Atmospheric Parameters}

In this section, we compare the physical parameters obtained from the spectroscopic and photometric methods described above. For this comparison, we exclude all objects with trigonometric parallax uncertainties larger than 20\% (24 objects) or with unreliable spectroscopic $\log g$ determinations (37 objects), as discussed in Section 3.1. We thus end up with a subsample of 158 white dwarfs with reliable trigonometric parallax and spectroscopic data, displayed as the hatched histograms in Figures 1 and 2 above.

The comparison of photometric and spectroscopic temperatures for all white dwarfs in our restricted sample of 158 objects is displayed in Figure 4, on a logarithmic scale. The agreement is excellent through the entire temperature range explored here. The difference between photometric and spectroscopic temperatures is explored more quantitatively in Figure 5 where we show the distribution of the absolute temperature differences, $\Delta T_{\text {eff }}=\left|T_{\text {phot }}-T_{\text {spec }}\right|$, measured in units of $\sigma$, which is defined as the combined photomet-

ric and spectroscopic uncertainties, $\sigma^{2} \equiv \sigma_{T_{\text {phot }}}^{2}+\sigma_{T_{\text {spec }}}^{2}$. These results indicate that among the 158 white dwarfs composing our reliable subsample, the two temperature measurements agree within $1 \sigma$ for $80 \%$ of the stars, and within $2 \sigma$ for $96 \%$ of the stars, which is more than satisfactory given that the values expected from a Gaussian error distribution (implicitly assumed here) are 68.3 and $95.5 \%$ at 1 and $2 \sigma$ confidence levels, respectively.

Furthermore, for some objects exhibiting differences larger than $1.5 \sigma$, the temperature values can be shown to be problematic due to various reasons. For instance, several objects for which $\Delta T_{\text {eff }}>1.5 \sigma$ correspond to suspected or confirmed double degenerate binaries: $0101+048\left(\Delta T_{\text {eff }}=1.66 \sigma\right), 0142+312\left(\Delta T_{\text {eff }}=2.92 \sigma\right), 0326-273\left(\Delta T_{\text {eff }}=3.20 \sigma\right), 1447-190$ $\left(\Delta T_{\text {eff }}=2.35 \sigma\right), 1606+422\left(\Delta T_{\text {eff }}=1.57 \sigma\right)$, and $2111+261\left(\Delta T_{\text {eff }}=2.29 \sigma\right)$. These objects will be analyzed and discussed in more detail in Section 4.4 following our test of the massradius relation. For the moment, we will only point out that the derived temperatures for these objects are not meaningful since they were obtained under the assumption of single stars. For three other white dwarfs, the photometric temperature is probably inaccurate. The most striking case is that of $0232+035\left(\Delta T_{\text {eff }}=3.57 \sigma\right)$, the hottest white dwarf in our sample as measured by the spectroscopic method: $T_{\text {spec }}=67,133 \mathrm{~K}$ (not shown in Figure 4). At such high temperature, the energy distribution in the optical and infrared portions of the spectrum is almost completely independent of the effective temperature, so the photometric method yields a value that is far too low, $T_{\text {phot }}=37,329 \mathrm{~K}$. In the cases of $0518+333\left(\Delta T_{\text {eff }}=\right.$ $1.89 \sigma)$ and $2351-368\left(\Delta T_{\text {eff }}=2.77 \sigma\right)$, we only have two photometric measurements in the optical (in addition to infrared photometry), so the corresponding photometric temperature is poorly constrained. Finally, for one object, $2105-820\left(\Delta T_{\text {eff }}=1.94 \sigma\right)$, the spectroscopic temperature is probably overestimated. This white dwarf is of particular interest regarding 
the results of our investigation of the mass-radius relation, and we thus defer our discussion of this object to Section 4.6 .

The radius and surface gravity values obtained from the photometric and spectroscopic methods, respectively, can be converted into mass using the mass-radius relations with $\mathrm{C} / \mathrm{O}$ cores and thick hydrogen layers (for hydrogen-rich stars) or thin hydrogen layer (for heliumrich stars) described in Section 4.2. We insist on the fact that the mass-radius relation is used here only to bring both measured quantities on an equal footing for comparison, and thus what follows in the present section is not part of our test of the mass-radius relation. The differences between these spectroscopic and photometric masses are shown as a function of effective temperature 3 in Figure 6 for our 158 reliable objects. On average, the spectroscopic and photometric masses appear to be consistent, with no obvious systematic trend. Both mass values agree within $0.1 M_{\odot}$ for $70 \%$ of the stars. However, it is clear that there are significant discrepancies, with differences as large as $\sim 0.3 M_{\odot}$ in both directions, and even more in some instances. Many of these discrepancies can be explained by the presence of double degenerate binary systems; in such cases the objects appear overluminous if analyzed with the photometric technique under the assumption of a single star, and the radius is thus overestimated and the photometric mass underestimated. This is illustrated in Figure 6, where the known systems are identified as filled red circles (these correspond to 0101+048, 0135-052, 0326-273, 1242-105, 1639+153, and 1824+040). Most other objects that have $M_{\text {spec }}-M_{\text {phot }}>0.1 M_{\odot}$ (including the two most discrepant points for which $\left.M_{\text {spec }}-M_{\text {phot }}>0.4 M_{\odot}\right)$ are suspected double degenerates that will be discussed in Section 4.4 (these correspond to 0126+101, 0142+312, 0311-649, 1130+189, 1418-088, 1447-190, $1606+422,2048+809$, and $2111+261)$. These objects are shown as dotted open circles in Figure 6. Some white dwarfs for which $M_{\text {spec }}-M_{\text {phot }}<0.1 M_{\odot}$, such as $0644+375$ and 2105-820, will also be investigated on a star by star basis in further sections of this work, where various scenarios that could account for the observed discrepancies are explored.

The cumulative mass distributions using both spectroscopic and photometric methods, regardless of the effective temperature, are displayed in Figure 7 in blue and red, respectively. Note that the mass distributions include here both DA and DB stars. The mean masses obtained using the two fitting techniques are entirely consistent, near $\sim 0.65 M_{\odot}$, and these values are comparable to other values reported in the literature. We note a more pronounced extended low-mass tail for the photometric approach, which results from the presence of the unresolved degenerate binaries discussed in the previous paragraph. We also note the smaller dispersion and the sharper peak of the mass distribution obtained from spectroscopy, most

\footnotetext{
${ }^{3}$ In this figure we adopt the spectroscopic or photometric effective temperature depending on criteria discussed in Section 4.1 .
} 


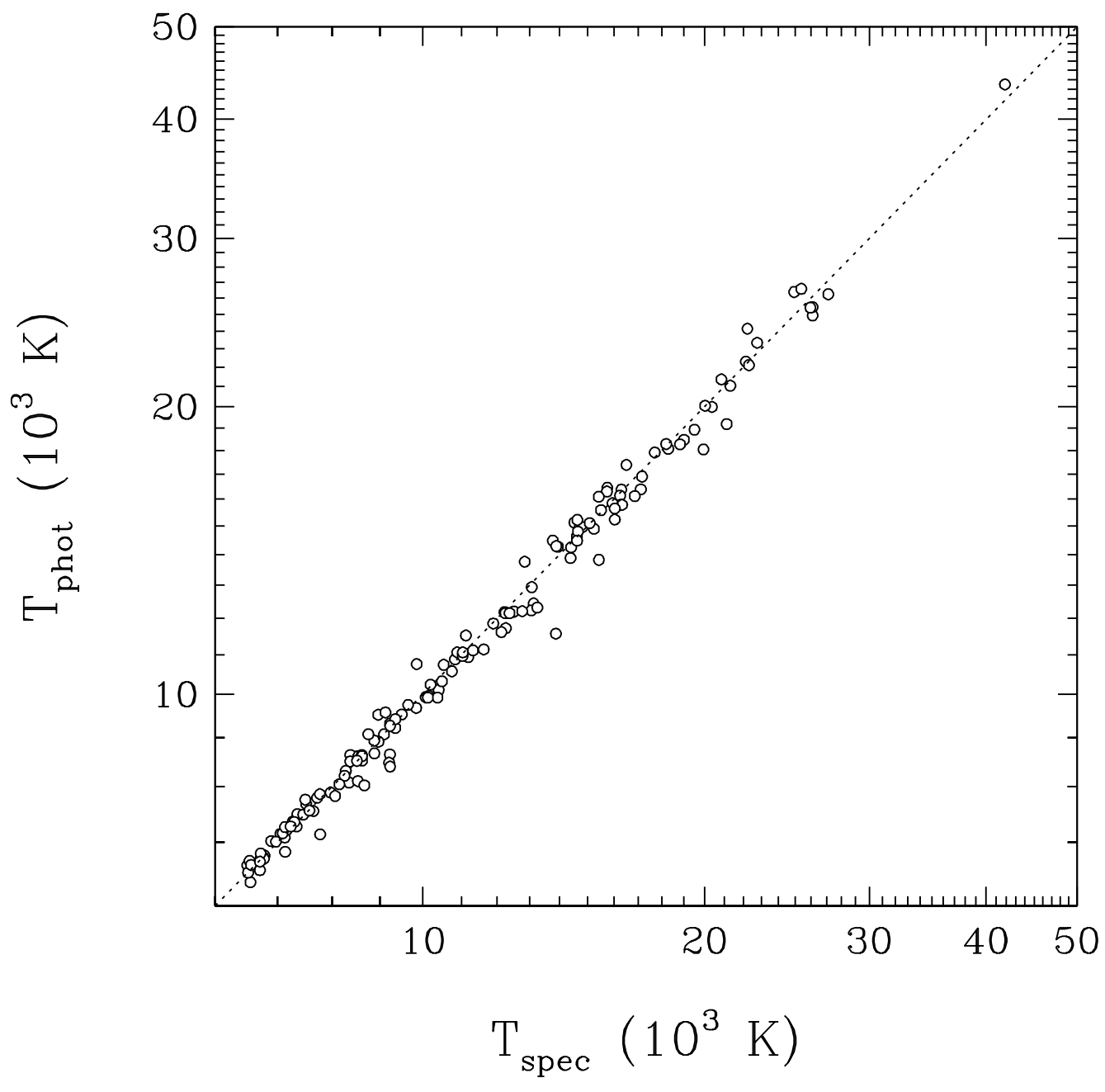

Fig. 4.- Comparison of photometric and spectroscopic effective temperatures for all white dwarfs in our sample with reliable trigonometric parallax and spectroscopic log g measurements. 


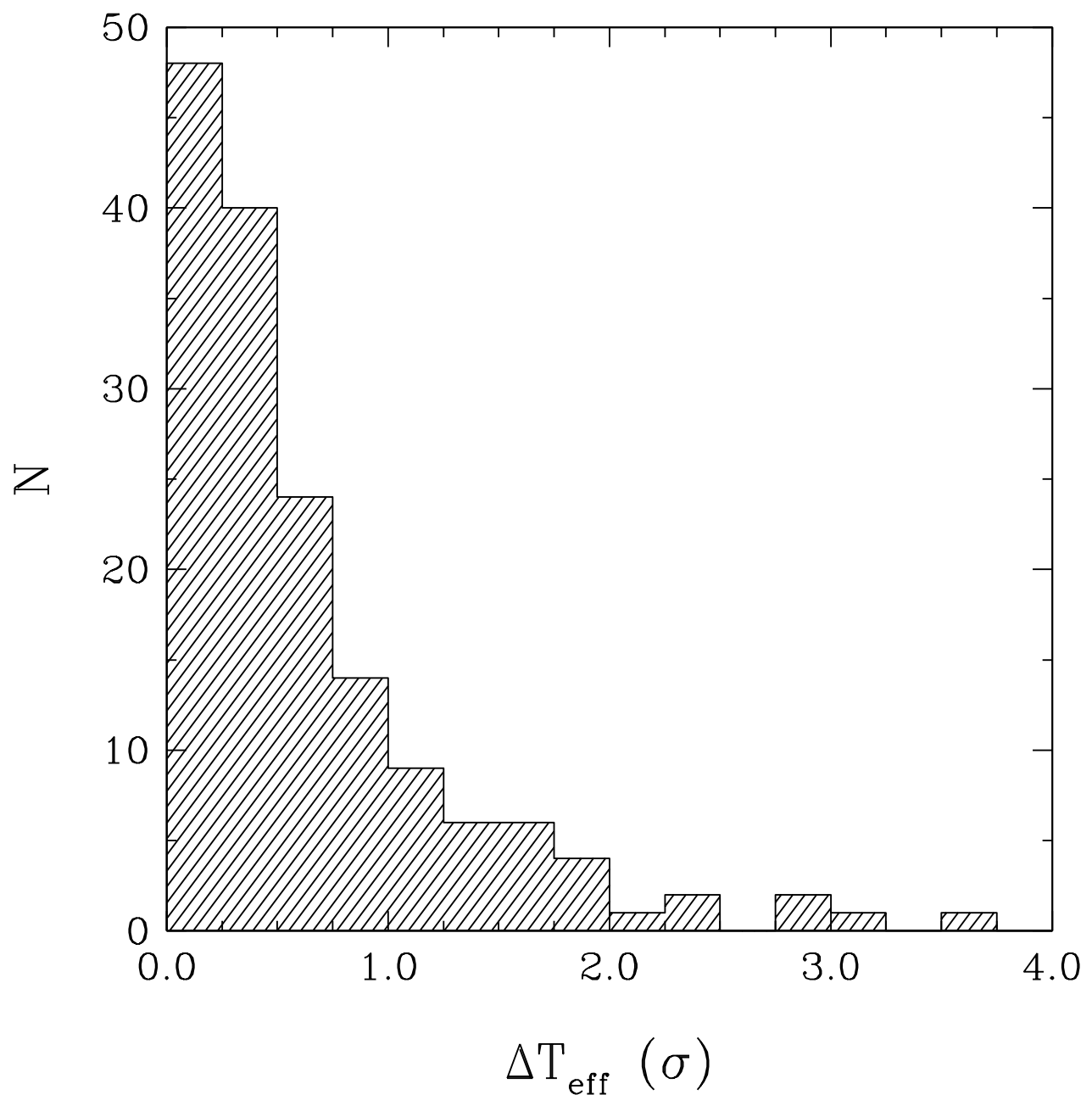

Fig. 5.- Distribution of the (absolute) differences between photometric and spectroscopic effective temperatures, measured in units of $\sigma$, where $\sigma^{2} \equiv \sigma_{T_{\text {phot }}}^{2}+\sigma_{T_{\text {spec }}}^{2}$, for all stars in our sample with reliable trigonometric parallax and spectroscopic $\log g$ measurements. 


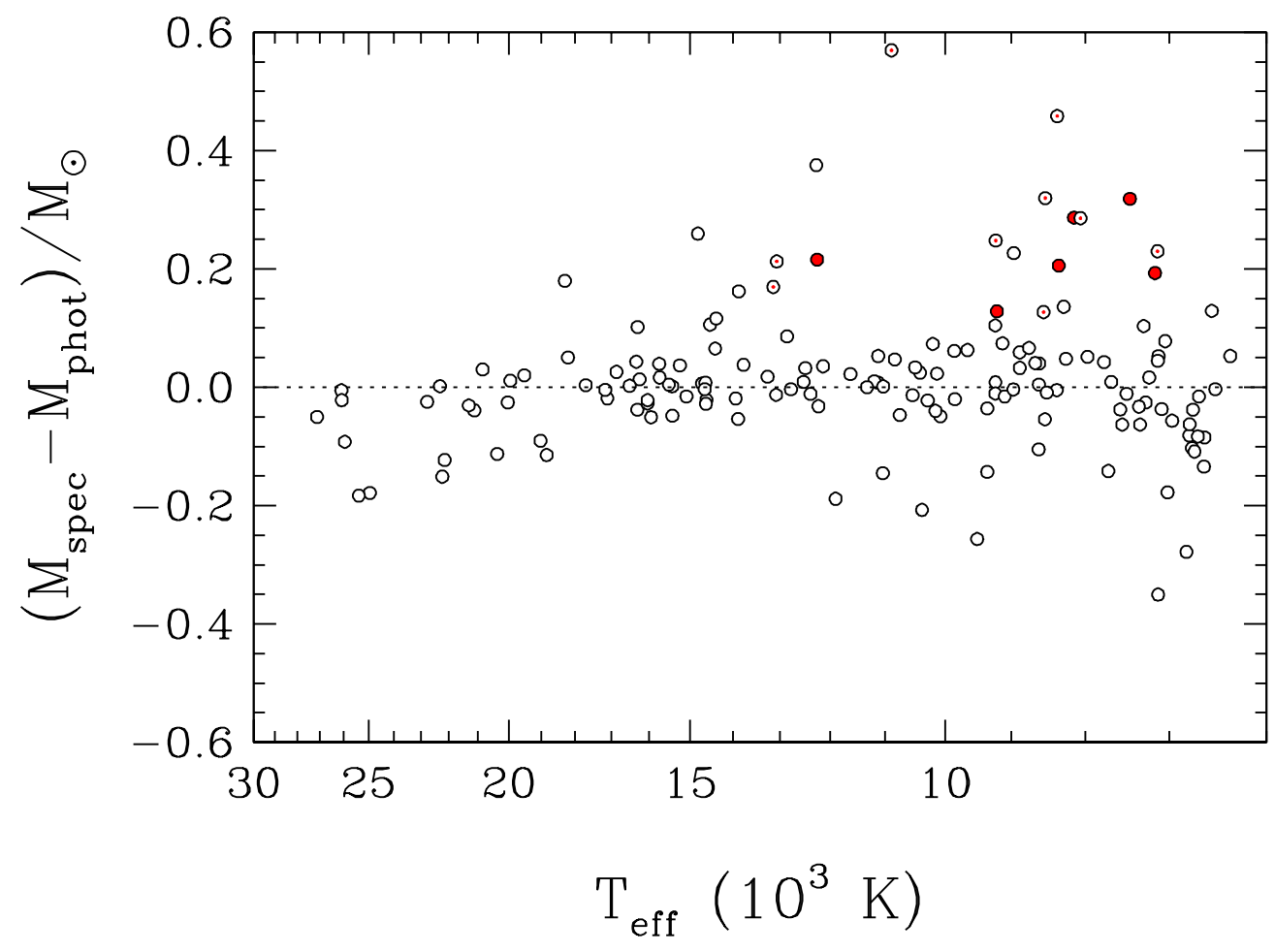

Fig. 6.- Differences in mass obtained from the spectroscopic and photometric methods as a function of effective temperature for all white dwarfs in our sample with reliable trigonometric parallax and spectroscopic log g measurements. The objects shown as filled red circles and dotted open circles correspond to confirmed and suspected double degenerate binaries, respectively. 


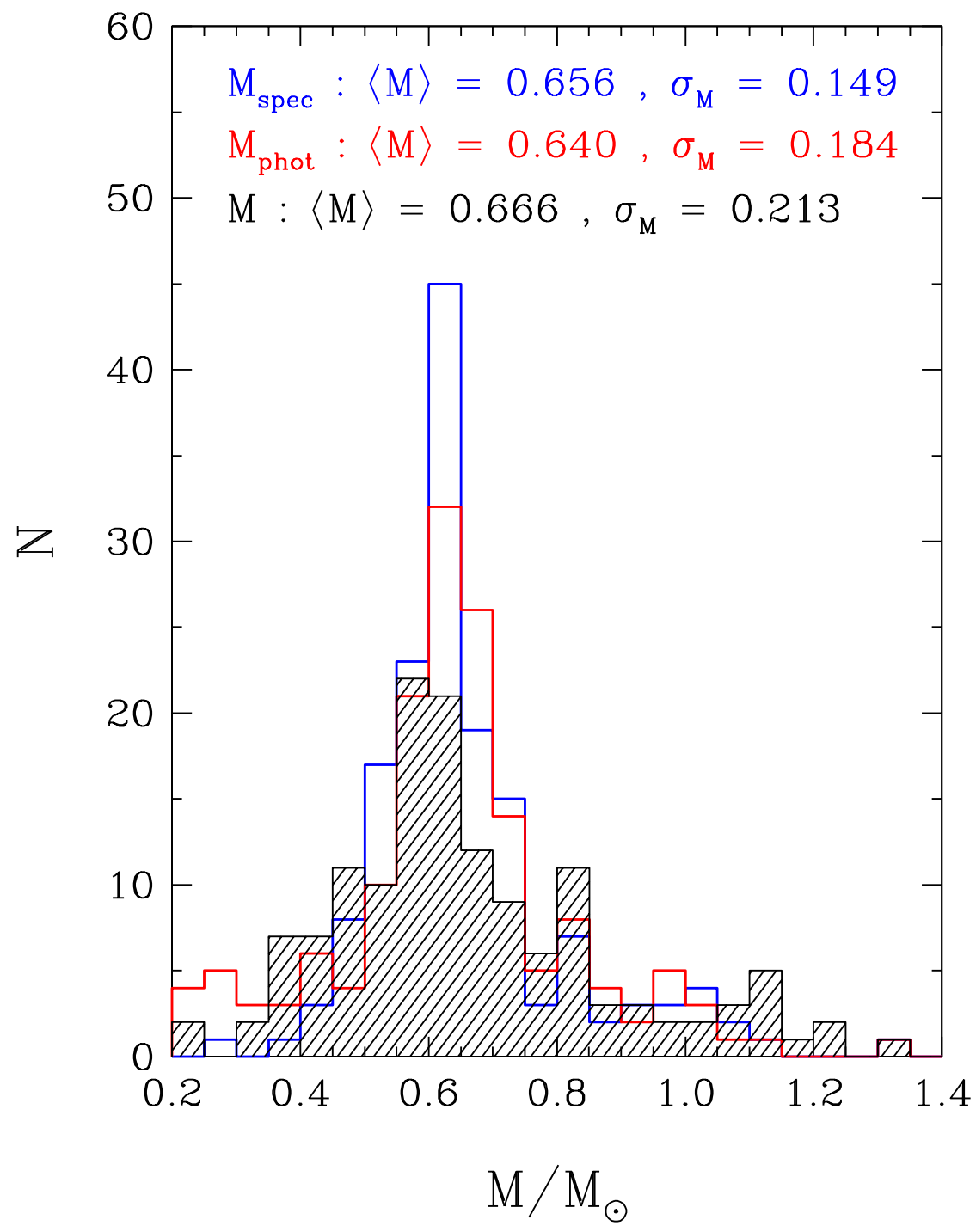

Fig. 7.- Comparison of the mass distributions obtained from the spectroscopic (blue) and photometric (red) methods for all white dwarfs in our sample with reliable trigonometric parallax and spectroscopic $\log g$ measurements. The mean masses and standard deviations, in $M_{\odot}$ units, are given in the figure. Also shown in black is the mass distribution obtained independently of the mass-radius relation (see Sections 4.1 and 4.8). 
likely because of the higher precision of the spectroscopic technique when applied to large ensembles of objects (but not necessarily for individual objects), and also because of the large trigonometric parallax uncertainties for some objects in our sample, which translate into photometric masses of low precision. The third mass distribution shown in black in the figure is obtained from the method employed to test the mass-radius relation and will be discussed later, in Section 4.8.

\section{TEST OF THE MASS-RADIUS RELATION}

\subsection{General Approach}

Our empirical test of the mass-radius relation relies on the combined use of the atmospheric and stellar parameters determined from both the spectroscopic and photometric techniques. The spectroscopic analysis, which provides values of $T_{\text {eff }}$ and $\log g$, constitutes the first step in our investigation, since the spectroscopic $\log g$ is needed as input for the calculation of the model fluxes in the photometric analysis, as outlined above. Then, the photometric technique is used to obtain the solid angle $\pi(R / D)^{2}$; this can be achieved in two different fashions: one can either consider both the effective temperature and the solid angle as free parameters during the fitting procedure (which yields an estimate of the photometric temperature), or one can set the effective temperature at the spectroscopic value and consider only the solid angle as a free parameter. This is illustrated in the top two panels of Figure 8 where we show our best photometric fits for the DA star 0644+375 (G87-7) as an example; in the first panel both $T_{\text {eff }}$ and $(R / D)^{2}$ are free parameters, while in the second panel $T_{\text {eff }}$ is forced to its spectroscopic value and only $(R / D)^{2}$ is a free parameter. (The full spectroscopic solution is also indicated as a reference at the top of the plot together with the source of the trigonometric parallax measurement.) For the majority of stars in our sample, we choose the latter solution and hence we adopt the spectroscopic temperature for the rest of our analysis, given our high level of confidence in the atmospheric parameters provided by the spectroscopic method. For some stars whose optical spectrum is noisy or shows weak features, we prefer to adopt the photometric temperature. However, it should be noted that this choice between the two approaches does not affect the resulting value of $(R / D)^{2}$ significantly, since the overall agreement between the spectroscopic and photometric temperatures is very good, as shown in Section 3.3 - a $0.7 \%$ difference in the case of G87-7. The color used in the two bottom panels of Figure 8, described in the next paragraph, reflects our choice of photometric (red) or spectroscopic (blue) temperature.

The mass-radius relation can be investigated using a method similar to that employed

by Provencal et al. (1998) for field white dwarfs. The value of $(R / D)^{2}$ is combined with the 
WD0644+375, G87-7 (DA)

Source of parallax : Hipparcos

Spectroscopic parameters : $\mathrm{T}_{\text {eff }}=22144 \mathrm{~K}, \log \mathrm{g}=8.09$
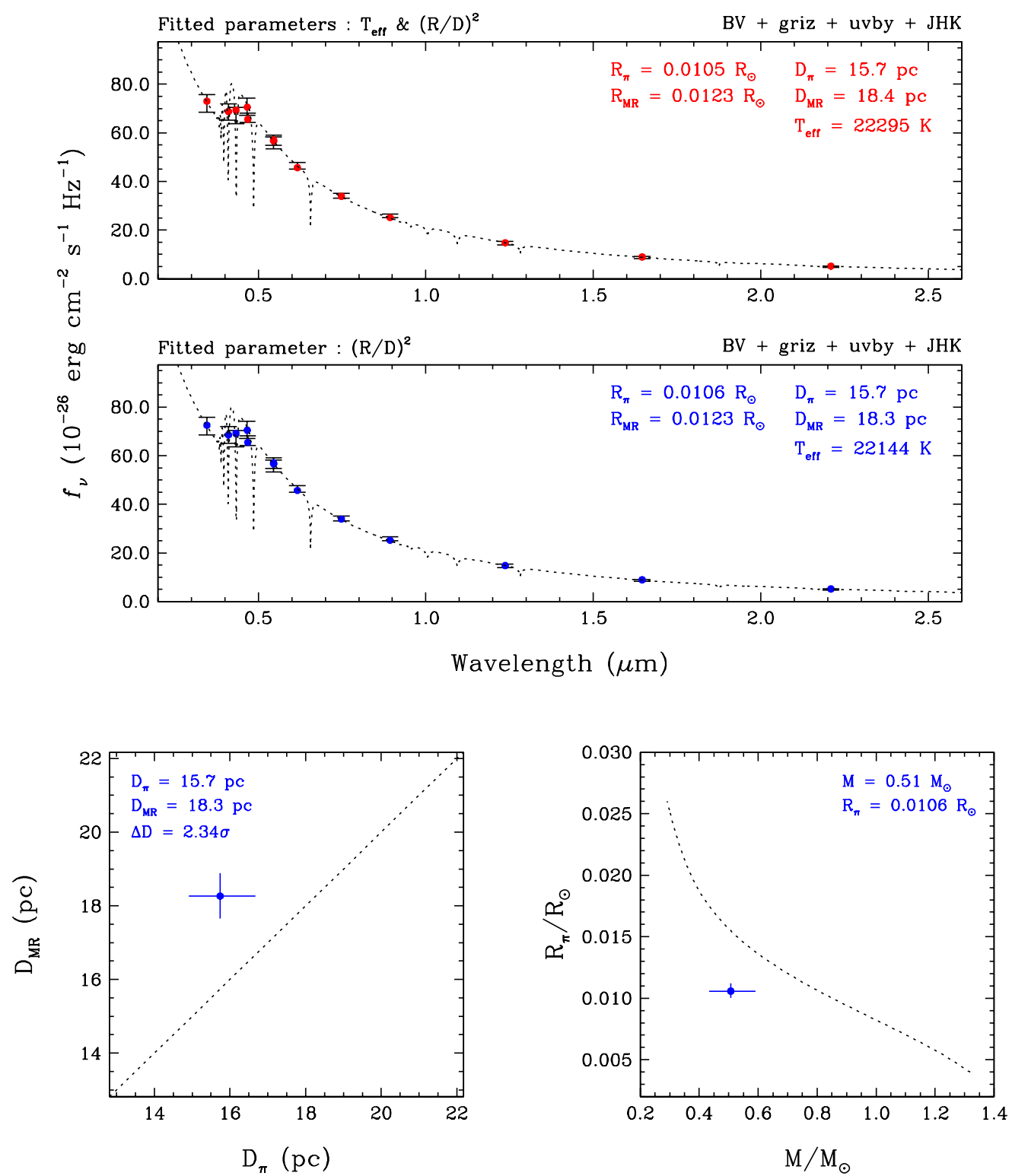

Fig. 8. - Top panels: Fits to the energy distribution of 0644+375 (G87-7), with the effective temperature considered as a free parameter (first panel, in red) or fixed at the spectroscopic value (second panel, in blue). Photometric observations are represented by error bars, while average model fluxes are displayed as filled circles. The monochromatic model flux is also shown as the dotted line. Bottom panels: Location of $0644+375$ (G87-7) in the $D_{\mathrm{MR}}$ versus $D_{\pi}$ diagram (left panel) and in the $R_{\pi}$ versus $M$ diagram (right panel). The dotted lines represent the 1:1 correspondence (left panel) and the mass-radius relation for $\mathrm{C} / \mathrm{O}$-core, thick hydrogen envelope models at the derived effective temperature (right panel). The color used in both plots indicates which of the photometric fits is adopted. 
star's distance from Earth, obtained directly from the trigonometric parallax, to derive the stellar radius (exactly as explained in Section [3.2), which we respectively label $D_{\pi}$ and $R_{\pi}$ in Figure 8 and other following figures. The surface gravity obtained from spectroscopy and the radius are then used to determine the stellar mass $M$ through the well-known relation $g=G M / R^{2}$. Thus, we end up with radius and mass estimates that are independent of any mass-radius relation. The derived values can then be compared to the predicted $R$ versus $M$ curve at a given effective temperature and for a specific core composition and hydrogen layer thickness. This is illustrated in the bottom right panel of Figure 8 for the particular case of G87-7, where we use the C/O-core mass-radius relations with thick hydrogen layers discussed in Section 4.2 below.

Another way to test the mass-radius relation is to compare the distance obtained from the parallax, $D_{\pi}$, to another estimate of the distance that depends on the mass-radius relation, which we denote $D_{\mathrm{MR}}$ in Figure 8 and subsequent figures. This distance is calculated by first using evolutionary models to convert the spectroscopic log $g$ into radius — labeled $R_{\mathrm{MR}}$ in Figure 8 - which is then combined with the photometric value of $(R / D)^{2}$ to obtain the desired distance $D_{\mathrm{MR}}$. This $D_{\mathrm{MR}}$ versus $D_{\pi}$ comparison, illustrated in the bottom left panel of Figure 8, has the advantage over the $R_{\pi}$ versus $M$ comparison that the x-axis (the parallax distance $D_{\pi}$ ) is a well-understood, model-independent quantity, for which the error bars simply originate from the parallax measurement uncertainty. Also, by comparing two estimates of the same quantity, the level of consistency can be easily evaluated in terms of the absolute difference between the two values expressed in units of the combined uncertainties $\sigma$, as was done in Section 3.3 for spectroscopic and photometric temperatures. This approach is explained further in Section 4.3.

As mentioned in Section [3.2, the uncertainties on $T_{\text {eff }}$ and the solid angle $\pi(R / D)^{2}$, measured using the photometric technique, are obtained directly from the covariance matrix of the Levenberg-Marquardt minimization method. The internal errors on $T_{\text {eff }}$ and $\log g$ using the spectroscopic technique are obtained in the same way, but these are combined in quadrature with the external errors, which for the moment we assume to be $1.4 \%$ in $T_{\text {eff }}$ and 0.042 dex in $\log g$, following Liebert et al. (2005). The uncertainties for all other quantities derived from these parameters - for instance the radius $R_{\pi}$ obtained from the solid angle combined with the measured trigonometric parallax - are calculated by propagating in quadrature the appropriate measurement errors (see also Holberg et al. 2012). These uncertainties, representing the $1 \sigma$ confidence level, are displayed as error bars in the bottom panels of Figure 8. In the particular case of G87-7, a significant $2.34 \sigma$ difference is observed between the distance obtained from the trigonometric parallax, $D_{\pi}=15.7 \pm 0.9 \mathrm{pc}$, and the distance obtained using the mass-radius relation, $D_{\mathrm{MR}}=18.3 \pm 0.6$ pc. Accordingly, G87-7 is located quite far from the mass-radius relation in the $R_{\pi}$ versus $M$ diagram of Figure 8 . 


\subsection{Mass-Radius Relations}

In a few of the results presented so far, and in the remainder of this analysis, we make extensive use of mass-radius relations using evolutionary models described at length in Fontaine et al. (2001) but with different core compositions and thicknesses of the hydrogen layers. Our reference stellar models for the DA stars in our sample have C/O-cores and "thick" hydrogen envelopes; that is, a core consisting of a uniform mixture of carbon and oxygen in equal proportions $\left(X_{\mathrm{C}}=X_{\mathrm{O}}=0.5\right)$ surrounded by a helium mantle of $q(\mathrm{He}) \equiv$ $M_{\mathrm{He}} / M_{\star}=10^{-2}$ and an outermost hydrogen layer of $q(\mathrm{H})=10^{-4}$. It is of interest to point out here that the mass-radius relation is not sensitive to the exact distribution of $\mathrm{C}$ and $\mathrm{O}$ in the core. For example, a standard 0.6 $M_{\odot}$ "thick" envelope DA model with a uniform $(50 / 50) \mathrm{C} / \mathrm{O}$-core composition has a total radius at $T_{\text {eff }}=15,000 \mathrm{~K}$ that is only $0.06 \%$ larger than the radius of an equivalent model, but with a detailed nonuniform $\mathrm{C} / \mathrm{O}$ stratification (with $X_{\mathrm{C}}=0.218$ and $X_{\mathrm{O}}=0.782$ at the center and becoming increasingly O-poorer in the above layers) obtained from the calculations of Salaris et al. (1997), which incorporate the effects of stellar evolution from the ZAMS. This difference in radius is a totally negligible effect in the present context.

We also make use of models with "thin" hydrogen envelope, identical to the thick models described above but with a much thinner hydrogen layer of only $q(\mathrm{H})=10^{-10}$, which are representative of hydrogen-atmosphere white dwarfs with thin hydrogen envelopes. Such a small layer of hydrogen does not change in any significant way the mass-radius relation with respect to models with no hydrogen, and from that point of view, that same mass-radius relation can also be used for helium-atmosphere white dwarfs. As discussed in BLR01, while it is possible with our code to compute evolutionary sequences with pure helium envelopes, the cooling times show an extreme sensitivity to the presence of even very small traces of heavy elements in the outer layers, leading to a continuum of ages, hence it is usually preferred to use models with a thin hydrogen layer for DB stars.

Additional models with a different core composition were calculated for the specific purpose of our analysis; these models have pure iron cores surrounded by helium and hydrogen layers identical to that of our thick models, i.e., $q(\mathrm{He})=10^{-2}$ and $q(\mathrm{H})=10^{-4}$. The essential input physics used in this context is the dense-plasma equation-of-state code developed by Lamb \& van Horn (1975) as appropriate for the fully ionized liquid/solid interior of a white dwarf. We used that code to compute the needed equation-of-state data for pure Fe. Those data largely specify the mass-radius relation of a model. Our Fe-core models will be used and discussed in Section 4.5 ,

In Figure 9, inspired from Figure 1 of Tremblay et al. (2017), we show the ratio of the radii obtained from our thin and thick envelope models (with our standard $\mathrm{C} / \mathrm{O}$-cores) as 


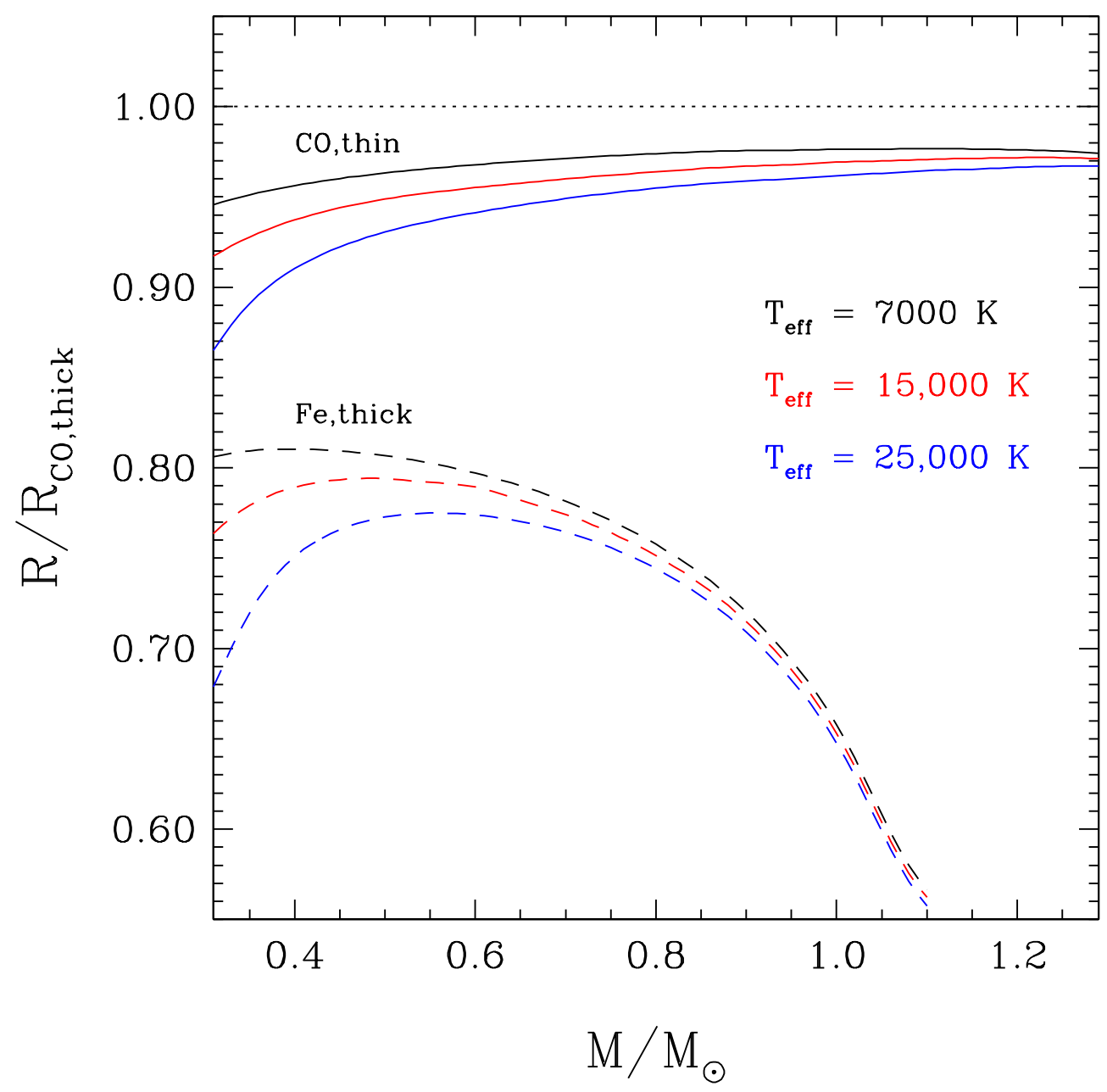

Fig. 9.- Ratio of the radii obtained from thin hydrogen envelope $\left(q(\mathrm{H})=10^{-10}\right)$ C/O-core models (solid lines) and from thick hydrogen envelope $\left(q(\mathrm{H})=10^{-4}\right)$ Fe-core models (dashed lines) with respect to our reference thick hydrogen envelope $\left(q(\mathrm{H})=10^{-4}\right) \mathrm{C} / \mathrm{O}$-core models, as a function of mass, for three representative values of effective temperature given in the figure. 
a function of mass for three values of effective temperature, which are representative of our white dwarf sample. Also shown in Figure9 is the same ratio but between the radii calculated from our Fe-core and $\mathrm{C} / \mathrm{O}$-core models (both with a thick hydrogen layer). As can be seen from these results, the thickness of the hydrogen layer has only a moderate effect $(\lesssim 5 \%)$ on the expected radius for normal $\sim 0.6 M_{\odot}$ white dwarfs, and above. A more important effect is observed for the core composition, which yields differences as large as $20 \%$ in radius for normal $\sim 0.6 M_{\odot}$ Fe-core white dwarfs with respect to our reference $\mathrm{C} / \mathrm{O}$-core models, and up to $40 \%$ at $1.0 M_{\odot}$. We also notice the importance of finite-temperature effects on these mass-radius relations, in particular for the low-mass models.

\subsection{Global Results}

The results for all white dwarfs in our sample — identical in format to those shown in Figure 8 for G87-7 — are provided as supplementary online figures, available on our Websitt4. If an object was rejected from our analysis on the basis of unreliable parallax or spectroscopic $\log g$ measurement, as discussed above, it is flagged with the label "R" placed in the upper right corner of the figure. Again, the particular color used in the two bottom panels indicates whether the photometric (red) or spectroscopic (blue) temperature is adopted in the photometric fit. The corresponding values of the parameters $D_{\pi}, D_{\mathrm{MR}}$, $M$ and $R_{\pi}$ are given in these two panels. In the $D_{\mathrm{MR}}$ versus $D_{\pi}$ diagram, we also give, as a quantitative measure of consistency with the $\mathrm{C} / \mathrm{O}$-core mass-radius relation, the value of the absolute difference between the two distance estimates, $\Delta D=\left|D_{\mathrm{MR}}-D_{\pi}\right|$, expressed in units of the combined uncertainties $\sigma$, defined as $\sigma^{2} \equiv \sigma_{D_{\mathrm{MR}}}^{2}+\sigma_{D_{\pi}}^{2}$.

The comparison of distance estimates is summarized in Figure [10 for the 158 white dwarfs with reliable trigonometric parallax and spectroscopic $\log g$ measurements in our sample. The 55 objects with distance differences larger than a $1 \sigma$ confidence level are displayed in red. Also, known unresolved degenerate binaries are shown as filled red symbols in the same figure; their location in this diagram suggests that some of the most discrepant results can probably be explained in terms of unresolved double degenerates, as noted in Section 3.3. We explore this scenario more quantitatively in Section 4.4 (the double degenerate candidates that will be identified and discussed in that section are displayed as dotted open circles in Figure 10).

We show in Figure 11, for the same reliable subsample, the distribution of the absolute differences in distances measured in units of $\sigma$, as defined above. We find that the fractions

\footnotetext{
4 http://www.astro.umontreal.ca/ bergeron/BedardApJ
} 


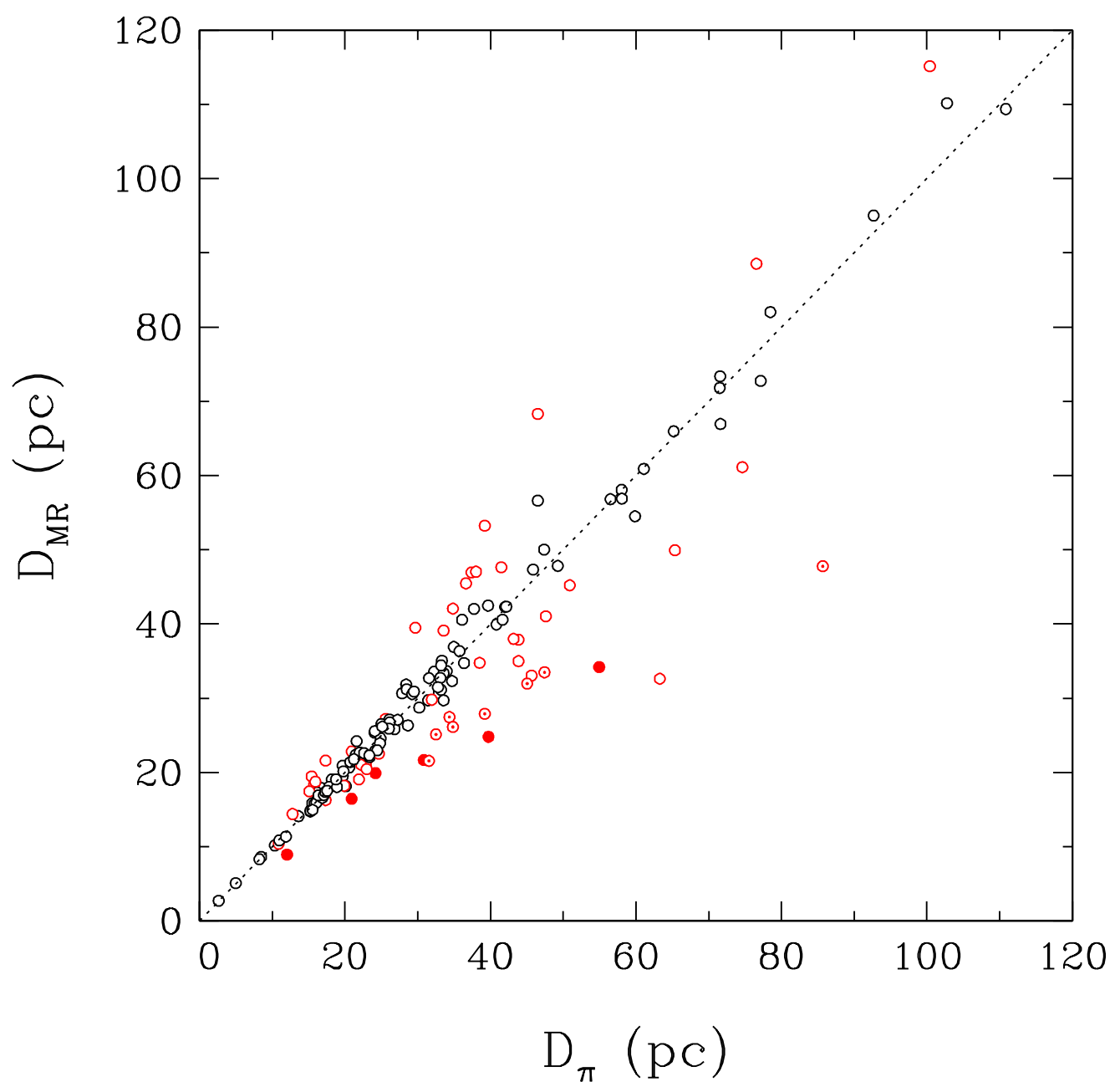

Fig. 10.- Comparison of the distances inferred from the mass-radius relation, $D_{\mathrm{MR}}$, with those obtained directly from the trigonometric parallax measurements, $D_{\pi}$, for all white dwarfs in our sample with reliable trigonometric parallax and spectroscopic log $g$ measurements (158 objects). The dotted line indicates the 1:1 correspondence. The stars shown in red ( 55 objects) exhibit differences larger than a $1 \sigma$ confidence level between the two distance estimates. The filled red circles and the dotted open circles represent, respectively, known and suspected unresolved double degenerate systems. 


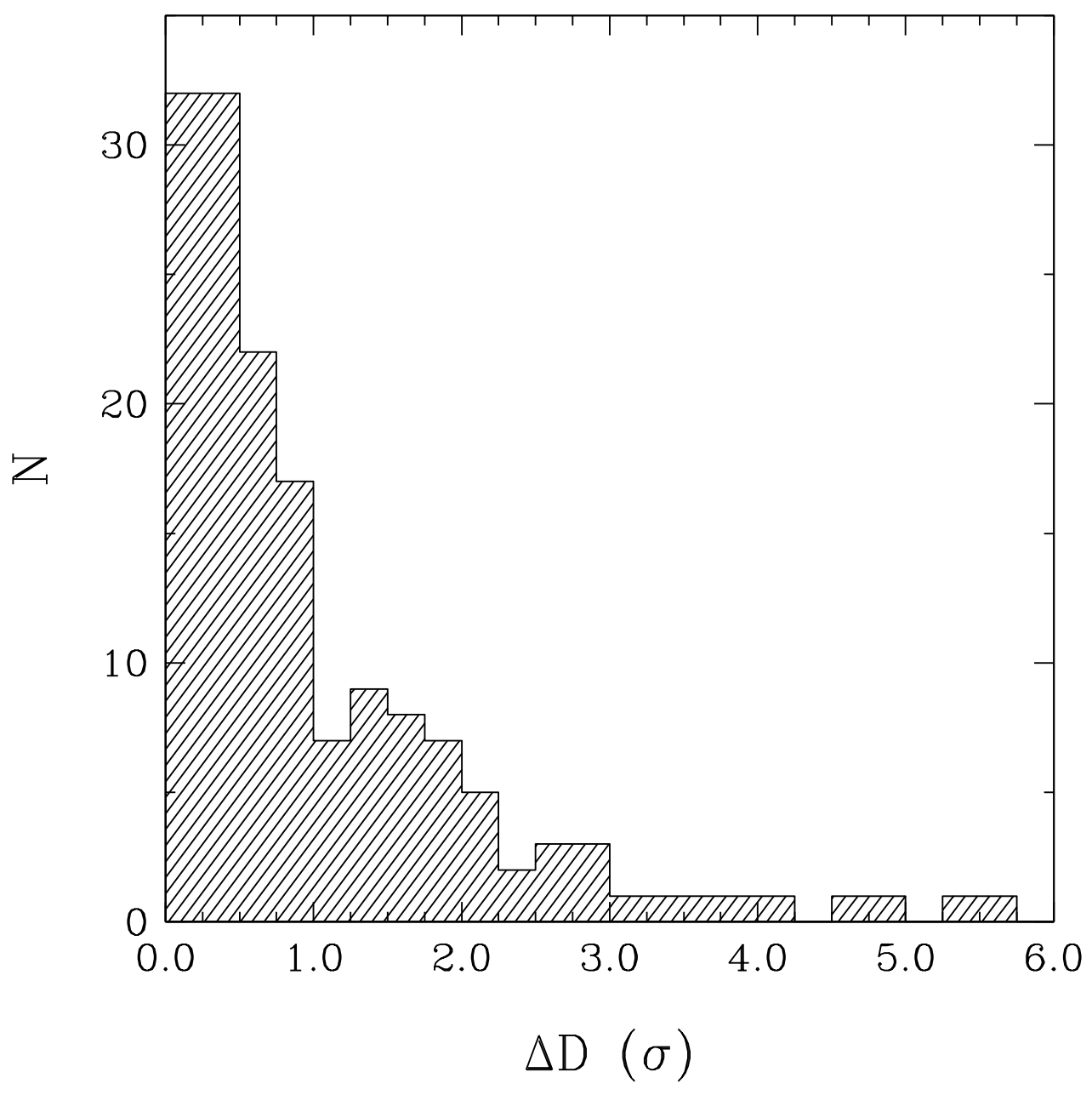

Fig. 11.- Distribution of the (absolute) differences between the distances $D_{\mathrm{MR}}$ and $D_{\pi}$, measured in units of $\sigma$, where $\sigma^{2} \equiv \sigma_{D_{\mathrm{MR}}}^{2}+\sigma_{D_{\pi}}^{2}$, for all white dwarfs in our sample with reliable trigonometric parallax and spectroscopic $\log g$ measurements. 
of white dwarfs having distance estimates within the 1 and $2 \sigma$ confidence levels are 65 and $85 \%$, respectively. These proportions are smaller than those expected from a Gaussian distribution, especially at the $2 \sigma$ level, for which our value departs from the standard $95.5 \%$ value by $\sim 10 \%$. Furthermore, for $7 \%$ of the stars, the distance differences are larger than $3 \sigma$, and thus the two distances are clearly statistically inconsistent. These results suggest that our sample contains a small number of outliers, which either have erroneous physical parameters, or simply do not follow the $\mathrm{C} / \mathrm{O}$-core mass-radius relation. We examine and discuss these two possibilities in the next few sections.

We also show, in the bottom panel of Figure 12, the differences in distances, $D_{\pi}-$ $D_{\mathrm{MR}}$ (expressed as fractions of the parallax distances $D_{\pi}$ ), but this time as a function of effective temperature. The results indicate that the general level of consistency does not vary significantly throughout the temperature range of our sample. Once again, the confirmed double degenerate systems occupy a well-defined region in the plot; in all six cases, $D_{\pi}$ is greater than $D_{\mathrm{MR}}$ by more than $15 \%$. Interestingly enough, had we neglected the hydrodynamical 3D corrections of Tremblay et al. (2013) applied to both our spectroscopic $T_{\text {eff }}$ and $\log g$ determinations for DA stars, severe discrepancies would have been present at low effective temperatures, where convective energy transport becomes important $\left(T_{\text {eff }} \lesssim\right.$ $13,000 \mathrm{~K}$ ), as can be seen in the upper panel of Figure 12. The comparison displayed here thus provides a strong support to the results of Tremblay et al., and in particular in the interpretation that the mixing-length theory is the culprit behind the so-called high-log $g$ problem observed in most spectroscopic analyses of DA stars (see Tremblay et al. 2010 and references therein).

The location of the same white dwarfs in the $R_{\pi}$ versus $M$ diagram is summarized in Figure 13 together with various theoretical mass-radius relations. We remind the reader that in this plot, the radius and mass estimates are independent of any mass-radius relation. The objects shown in red in this figure correspond to the same objects also shown in red in Figure 10, that is, white dwarfs that have $\Delta D>1 \sigma$. In particular, the unresolved double degenerate binaries all appear here on the right of the theoretical mass-radius relations. The object shown in black near the top of the figure, $0501+527$ (G191-B2B), is within $1 \sigma$ despite being located far on the right of the predicted curves, because the parallax measurement has a $17.8 \%$ uncertainty, leading to uncertainties on the radius and mass that are particularly significant for this hot star (see online figures). As can be seen from this plot, the possibility to ever measure the thickness of the hydrogen layer in DA white dwarfs using such diagrams is almost hopeless, because the difference between thin and thick hydrogen envelope models is way too small. This is particularly true for 40 Eri B (0413-077), which fits better the thin hydrogen envelope $\mathrm{C} / \mathrm{O}$-core models, but which is also consistent with the thick models at the $1 \sigma$ level, a conclusion also reached by Tremblay et al. (2017). The core composition, 


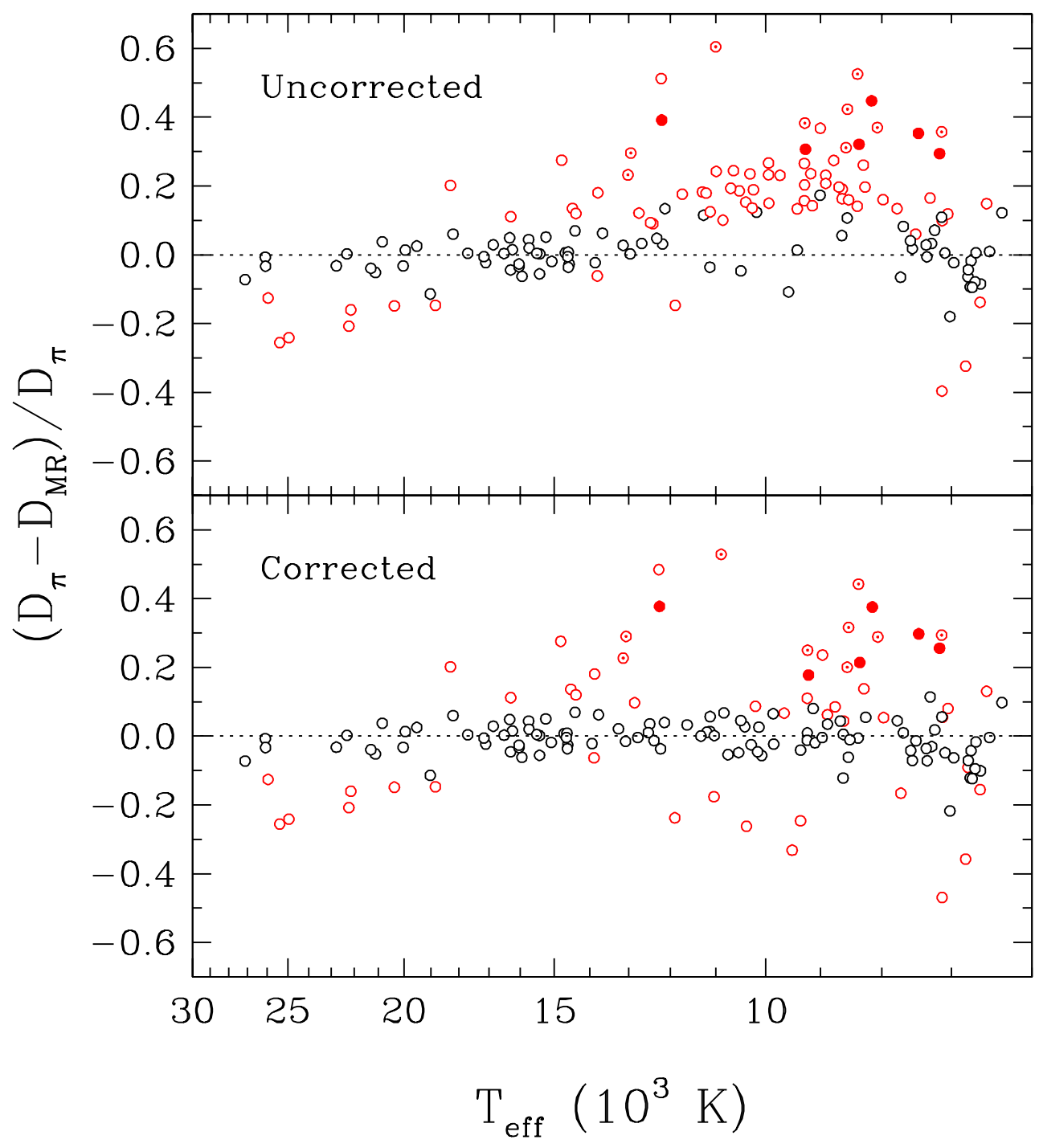

Fig. 12.- Differences between the distances $D_{\pi}$ and $D_{\mathrm{MR}}$, expressed as fractions of the parallax distance $D_{\pi}$, for all white dwarfs in our sample with reliable trigonometric parallax and spectroscopic $\log g$ measurements, as a function of effective temperature; the color coding is identical to that used in Figure 10. The results in the upper panel (uncorrected) do not take into account the hydrodynamical 3D corrections of Tremblay et al. (2013) for both spectroscopic $T_{\text {eff }}$ and $\log g$ determinations. 
however, may be within our reach if a white dwarf possesses a heavy core made of iron.

A small trend can also be observed in Figure 13, where most points seem to be roughly distributed along some diagonal pattern, from the lower left to the upper right. In this diagram, errors on the spectroscopic $\log g$ values would result in a horizontal rather than a diagonal pattern since only the mass $M$ depends on $\log g$. Instead, the observed feature most likely originates from inaccuracies on the radius determinations. Indeed, the net effect of a change in radius is to move a point diagonally since $R_{\pi}$ appears explicitly on the y-axis, but is also used in the calculation of $M$ on the x-axis. Since $R_{\pi}$ is obtained from the solid angle and the parallax measurement, and given that the solid angle is a very robust quantity derived from the energy distribution, the trend observed in Figure 13 can most naturally be explained by the distribution of errors in the parallax measurements. The Gaia mission should help to reduce the observed scatter in the $R_{\pi}$ versus $M$ diagram.

Among the stars that were rejected from our reliable subsample (the objects labeled " $\mathrm{R}$ " in the online figures), 24 have been excluded on the basis of parallax uncertainties larger that $20 \%$, and 37 because of unreliable spectroscopic $\log g$ measurements. We note, however, that despite the high uncertainty associated with these objects, some of them are still consistent with the expected mass-radius relation; in some cases, the agreement is even very good. For instance, 0221+399, 0230-144, 0655-390, 1630+189, 1710+683, 1811+327B, and 2347+292 are all cool objects $\left(T_{\text {eff }} \lesssim 6500 \mathrm{~K}\right)$ that were rejected on the basis of potentially unreliable $\log g$ determinations, but they all accurately match the predictions of the mass-radius relation (see online figures). This suggests that in some cases, the spectroscopic technique can provide reliable $\log g$ values even at such low effective temperatures. The spectroscopic fits for some of these cool white dwarfs are displayed in Figure 3 above. Furthermore, among the stars excluded because of uncertain parallaxes, 0145-174, 0407+179, 1244+149, 1425+540, $1518+636,1811+327 \mathrm{~A}, 2222+683$, and $2329+407$ all fall remarkably close to the expected curves (see bottom panels of the corresponding online figures), implying that the parallax measurements for these objects are probably accurate even if they have large uncertainties. Finally, we have to mention the peculiar case of $1132+470$ (G122-31), which was rejected because of a $34.5 \%$ parallax uncertainty. For this white dwarf, the temperature estimates obtained from spectroscopy and photometry are radically different, $T_{\text {spec }}=28,088 \mathrm{~K}$ and $T_{\text {phot }}=14,780 \mathrm{~K}$. This suggests that this object might actually be an unresolved double degenerate binary, most likely a DA+DC, an idea further supported by the dubious quality of our spectroscopic fit (shown in Figure 3), especially at H $\beta$. Harris et al. (2013) actually mention in their introduction the detection of a companion to G122-31, although these results have not been published yet.

In the remainder of this paper, we investigate the objects showing distance differences 


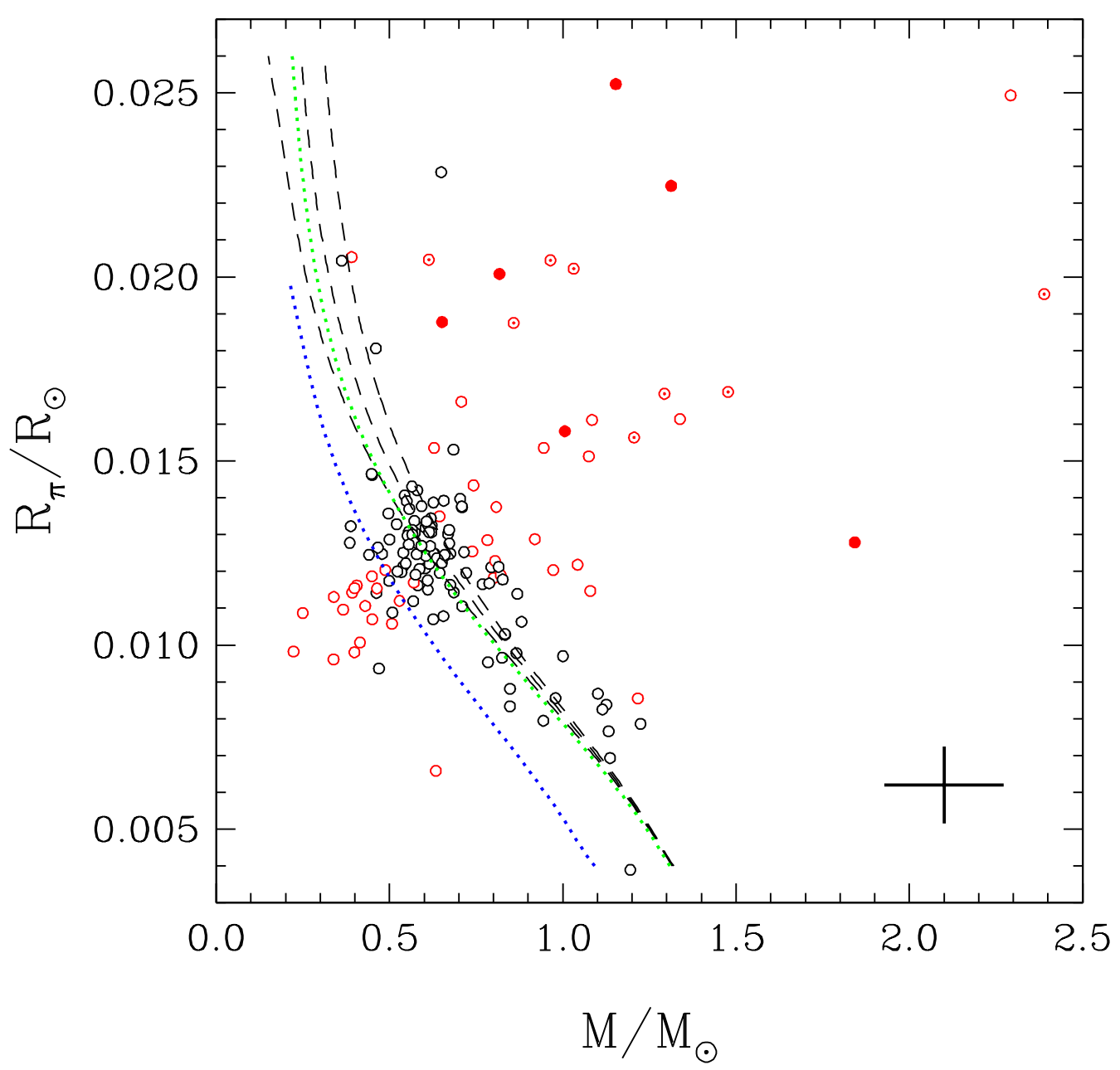

Fig. 13.- Location in the $R_{\pi}$ versus $M$ diagram for all white dwarfs in our sample with reliable trigonometric parallax and spectroscopic $\log g$ measurements; the color coding is identical to that used in Figure 10. The cross in the lower right corner represents the average uncertainties. Also shown are mass-radius relations for $\mathrm{C} / \mathrm{O}$-core, thick hydrogen envelope models at $T_{\text {eff }}=7000,15,000$, and 25,000 K (black dashed lines, from left to right), for C/O-core, thin hydrogen envelope models at $T_{\text {eff }}=15,000 \mathrm{~K}$ (green dotted line), and for Fe-core, thick hydrogen envelope models also at $T_{\text {eff }}=15,000 \mathrm{~K}$ (blue dotted line). 
larger than $1.5 \sigma$, with the aim of determining whether these discrepancies have a physical rather than a statistical origin. We put forward various physical interpretations that may explain the measured differences, and thus improve the agreement with the theoretical massradius relation for these stars.

\subsection{Double Degenerate Binaries}

\subsubsection{Confirmed and Suspected Double Degenerate Binaries}

Our sample contains six well-known unresolved double degenerate binaries, which are indicated by filled red circles in all the previous plots: 0101+048 (G1-45; Maxted et al. 2000; Farihi et al. 2005), 0135-052 (L870-2; Saffer et al. 1988; Bergeron et al. 1989), 0326-273 (L587-77A; Zuckerman et al. 2003; Nelemans et al. 2005), 1242-105 (LP 736-4; Debes et al. 2015), 1639+153 (LHS 3236; Harris et al. 2013), and 1824+040 (Ross 137; Maxted \& Marsh 1999; Morales-Rueda et al. 2005; Farihi et al. 2005). As expected, the location of all these binaries in our various diagrams is at odds with the predictions from the theoretical massradius relations. When analyzed under the assumption of a single star, an unresolved double degenerate binary will appear overluminous; the photometric fit will then overestimate the radius $R_{\pi}$, and also underestimate the distance $D_{\mathrm{MR}}$, as observed in Figures 10, 12, and 13, In particular, these six confirmed binaries show significant discrepancies in their distance estimates $(\Delta D=2.52,5.36,4.09,9.42,7.69$, and $2.90 \sigma$, respectively).

A close inspection of the various results presented in Section 4.3 reveals that 12 additional white dwarfs in our sample exhibit the typical discrepancies expected from unresolved degenerate binaries. Among these, five have already been identified as unresolved double degenerate candidates by BLR01: 0126+101 $(\Delta D=1.81 \sigma), 0142+312(\Delta D=1.61 \sigma)$, $1418-088(\Delta D=1.81 \sigma), 1606+422(\Delta D=1.78 \sigma)$, and $2111+261(\Delta D=2.60 \sigma)$. Our own results thus reinforce their conclusion about the likely binary nature of these white dwarfs. However, we find in our analysis that two additional double degenerate candidates identified by BLR01 are within the $1 \sigma$ confidence level assuming a single star: 0839-327 $(\Delta D=0.63 \sigma)$ and 1124-293 (named 1124-296 in BLR01; $\Delta D=0.67 \sigma$ ). In both cases, BLR01 based their conclusion on the fact that these white dwarfs appeared overluminous, resulting in extremely low photometric masses of $M=0.44 M_{\odot}$ and $M=0.23 M_{\odot}$, respectively. In the case of 0839-327, we also obtain a low mass of $M=0.45 M_{\odot}$, but its location right on the expected mass-radius relation (see online figures) suggests it is a single low-mass white dwarf, or if there is a companion, its contribution to the total luminosity of the system is negligible. For 1124-293, we obtain a normal mass of $M=0.54 M_{\odot}$, resulting from a more recent and more precise trigonometric parallax measurement, $\pi=31.00 \pm 1.54$ mas (Subasavage et al. 2017), 
a value which is twice as large as that used by BLR01 in their analysis, $\pi=16.4 \pm 1.7$ mas (Ruiz 1996). Again, the good agreement with the mass-radius relation shown in the online figures suggests that 1124-293 is a single DA white dwarf.

For two other white dwarfs in our sample, $1130+189(\Delta D=3.24 \sigma)$ and $2048+809$ $(\Delta D=4.77 \sigma)$, our results agree with those of Tremblay et al. (2017), who found large inconsistencies between the observed and predicted radii (the equivalent of $R_{\pi}$ and $R_{\mathrm{MR}}$ in our analysis). Hence, we concur with their interpretation that these stars are probably unresolved double degenerate binaries. Also, one object in our sample, 1447-190 $(\Delta D=2.95 \sigma)$, was recently identified as a double degenerate binary by Subasavage et al. (2017), who not only obtained discrepant distance estimates, but also reported radial velocity variations at $\mathrm{H} \beta$. Another possible double degenerate is $0311-649(\Delta D=4.53 \sigma)$, also studied by Subasavage et al., who suggested a binary hypothesis based on their low photometric mass $(M=0.29$ $\left.M_{\odot}\right)$.

The three remaining stars in our sample that could possibly be explained in terms of unresolved double degenerate binaries are 0133-116 $(\Delta D=3.73 \sigma), 0518+333(\Delta D=$ $1.58 \sigma)$, and $2351-368(\Delta D=1.54 \sigma)$. However, we have reasons to believe that these objects are not double degenerate systems. In the cases of $0518+333$ and $2351-368$, the energy distributions are poorly constrained and the parallax uncertainties are quite large (14.4 and $11.2 \%$, respectively), and hence more reliable data are needed before any definitive conclusion can be drawn. The puzzling case of 0133-116 (Ross 548, ZZ Ceti itself) requires a more extensive discussion, which we present in the next section.

Thus, besides the six known systems, we identified nine additional double degenerate candidates based on our analysis, which are shown as dotted open circles in previous plots.

\subsubsection{Atmospheric Parameters}

To strengthen our double degenerate interpretation for some of the objects in our sample, we present here a method to derive the atmospheric parameters of both components in unresolved degenerate binary systems. Our approach relies on modified versions of the spectroscopic and photometric techniques, and assumes that the theoretical mass-radius relation is valid for individual white dwarfs. Since all the confirmed and suspected binaries identified in our analysis are hydrogen-rich objects, we assume in the following that they are composed of two DA stars.

The monochromatic flux received at Earth from an unresolved double degenerate system is given by the sum of the Eddington flux originating from each component, properly weighted 
by their solid angle (see Equation 10):

$$
f_{\lambda}=\frac{4 \pi}{D^{2}}\left[R_{1}^{2} H_{\lambda, 1}\left(T_{\mathrm{eff}, 1}, \log g_{1}\right)+R_{2}^{2} H_{\lambda, 2}\left(T_{\mathrm{eff}, 2}, \log g_{2}\right)\right]
$$

where we have explicitly written the dependence of the Eddington flux on the atmospheric parameters. The flux received at Earth thus seems to depend on seven physical quantities the radius, effective temperature, and surface gravity of each component, and the distance but the mass-radius relation, which relates $R$ to $\log g$, reduces the number of free parameters to only five: $T_{\text {eff }, 1}, \log g_{1}, T_{\text {eff }, 2}, \log g_{2}$, and $D$.

With the definition of the combined flux given by Equation 2, the spectroscopic technique can be applied directly as described in Section 3.1, but this time by considering $T_{\text {eff } 1}$, $\log g_{1}, T_{\text {eff } 2}$, and $\log g_{2}$ as free parameters. Note that the factor $4 \pi / D^{2}$ in Equation 2 is irrelevant here since the spectroscopic technique relies on the normalized Balmer line profiles. A similar approach can be used with the photometric technique by averaging the combined fluxes over the appropriate filter bandpasses. However, since the photometric technique is based on absolute fluxes, the factor $4 \pi / D^{2}$ must be taken into account when comparing to observed fluxes, with the distance $D$ obtained from the trigonometric parallax. The fit to the energy distribution thus yields the same four atmospheric parameters as the spectroscopic technique. Note that even though the individual energy distributions depend only weakly on $\log g$, as mentioned in Section 3.2, the combined fluxes — as expressed by Equation 2 - are strongly dependent on the stellar radius $R$ of each component of the system, and thus on the corresponding $\log g$ values through the mass-radius relation. As such, both $\log g$ values obtained from the photometric fit are meaningful. Given the large number of fitting parameters, the application of one technique yields many different solutions, depending on the number of free and fixed parameters, as well as on the adopted initial values. However, very few solutions (or in many cases, only one solution) are consistent with both the spectroscopic and the photometric analyses. A consistent solution can thus be obtained by using the two techniques in an iterative way.

As a test case, we applied our fitting procedure to 0037-006, a known double-lined degenerate binary (Koester et al. 2009), which is not included in our sample because no parallax measurement is available for this star. In such a case, the distance $D$ is considered an additional free parameter in the photometric fit. This particular object was selected because high-resolution spectroscopy at $\mathrm{H} \alpha$ can be used to confirm that the model spectrum predicted from the four-atmospheric parameter solution reproduces reasonably well the observed double-lined core. Figure 14 shows the solution that best reproduces the spectroscopic and photometric observations for 0037-006. Also shown in the right panel is the 
contribution of each component (in red and blue) to the total flux (in black). The fit to both the Balmer line profiles and the energy distribution is excellent. We show in Figure 15 the superposition of the observed and model spectra at $\mathrm{H} \alpha$ for this particular solution (a wavelength shift between both components has been included in our solution to match to observed shift). We emphasize that this is not a fit to the $\mathrm{H} \alpha$ line profile. The double-lined feature is perfectly reproduced, which strengthens our confidence in our overall procedure to obtain reliable physical parameters for white dwarfs in unresolved binary systems.

Our fitting technique was applied to the 15 confirmed or suspected double degenerate binaries identified in our analysis, the results of which are displayed in Figure 16. For most of these objects, values of the atmospheric parameters of both components are reported for the first time. Even if our test case example for 0037-006 indicates that the atmospheric parameters obtained with our fitting procedure appear reliable, the quantitative results presented in Figure 16 must be interpreted with caution. In some instances, there are more than one acceptable solution, which is perhaps not surprising given the numerous free parameters. It is also possible that some of the objects are actually DA+DC systems rather than DA+DA systems, in which case the true atmospheric parameters probably differ from those derived here. Still, our method is able to demonstrate, undeniably, whether or not a white dwarf can be fitted as a double degenerate, at least in a qualitative way. We finally note that other estimates obtained from various methods are available in the literature for five well-known double degenerates (0135-052, 0326-273, 1242-105, 1639+153, and 1824+040), but we are refraining from making a detailed comparison since our goal here is only to demonstrate that these are double degenerate systems.

An excellent counterexample is provided by 0133-116 (Ross 548). Inconsistencies in the parameters of this white dwarf were first noticed by Bergeron et al. (1995b, see also Gianninas et al. 2011); our own analysis of this object presented in the online figures shows a similar behavior. The parallax measurement gives a distance $D_{\pi}=63.3 \pm 8.1 \mathrm{pc}$, which is twice as large as the distance computed by invoking the mass-radius relation, $D_{\mathrm{MR}}=$ $32.5 \pm 1.1$ pc. Similarly, we obtain a large radius, $R_{\pi}=0.0250 R_{\odot}$, which translates into a ridiculously high mass of $M=2.30 M_{\odot}$ for the measured spectroscopic value of $\log g=8.01$. Given that the spectroscopic fit is excellent (see Figure 3) and yields a normal log $g$ value, the inconsistency seems to arise instead from the photometric fit, as reflected by the high value of $R_{\pi}$ and the low value of $D_{\mathrm{MR}}$. This strongly suggests that Ross 548 might be a double degenerate system. However, the application of our fitting method to this object proved unsuccessful; there is just no composite model that can fit both the spectroscopy and the photometry simultaneously. In particular, for all acceptable sets of effective temperatures, the surface gravities derived from the Balmer line profiles differ from those obtained from the energy distribution by more than an order of magnitude. The reason for this disparity is that 


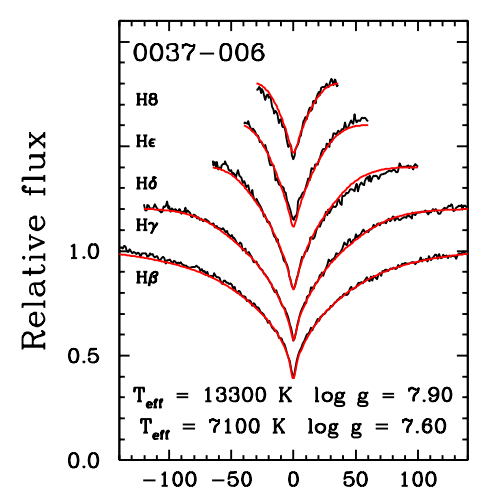

$\Delta \lambda(\AA)$

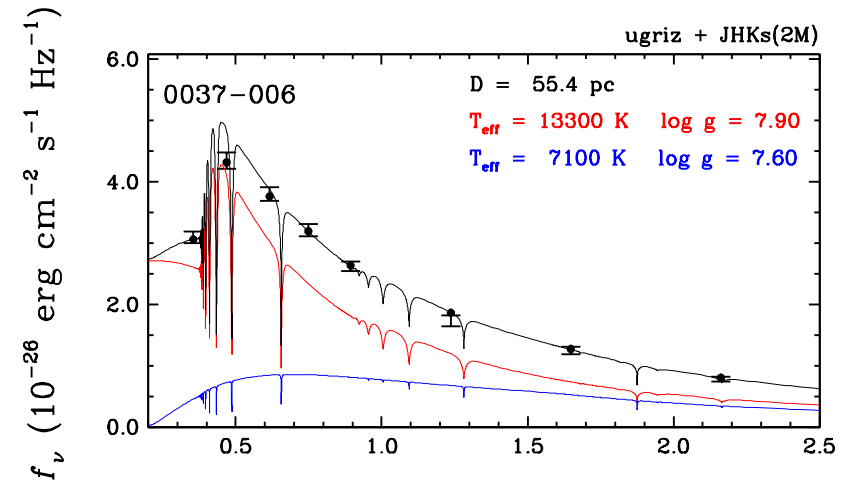

$\lambda(\mu \mathrm{m})$

Fig. 14.- Best fit to the optical spectrum (left panel) and energy distribution (right panel) of 0037-006, assuming an unresolved double degenerate system composed of two DA stars. In the right panel, the red and blue lines show the contribution of each component to the total model flux, which is displayed as the black line.

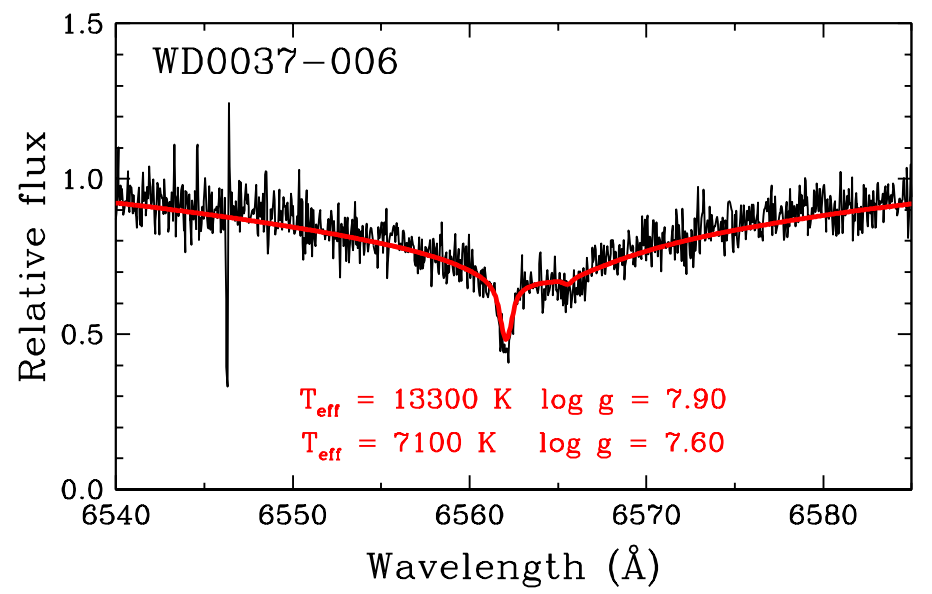

Fig. 15. - Superposition of the observed and model spectra of 0037-006 at H $\alpha$, assuming an unresolved double degenerate system composed of two DA stars whose atmospheric parameters correspond to the best solution shown in Figure 14. 


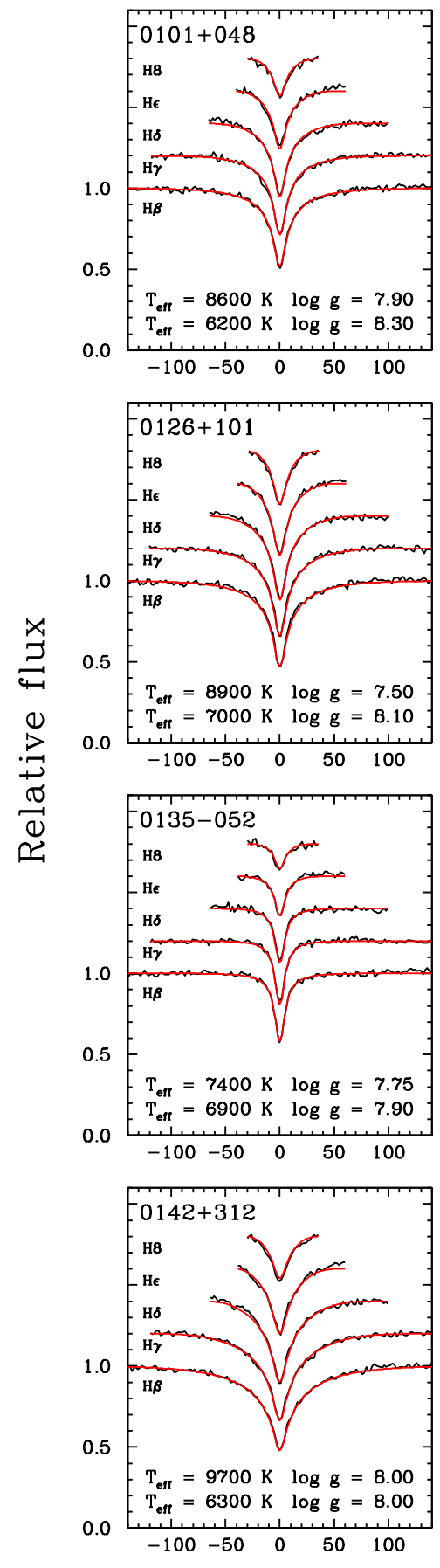

$\Delta \lambda(\AA)$
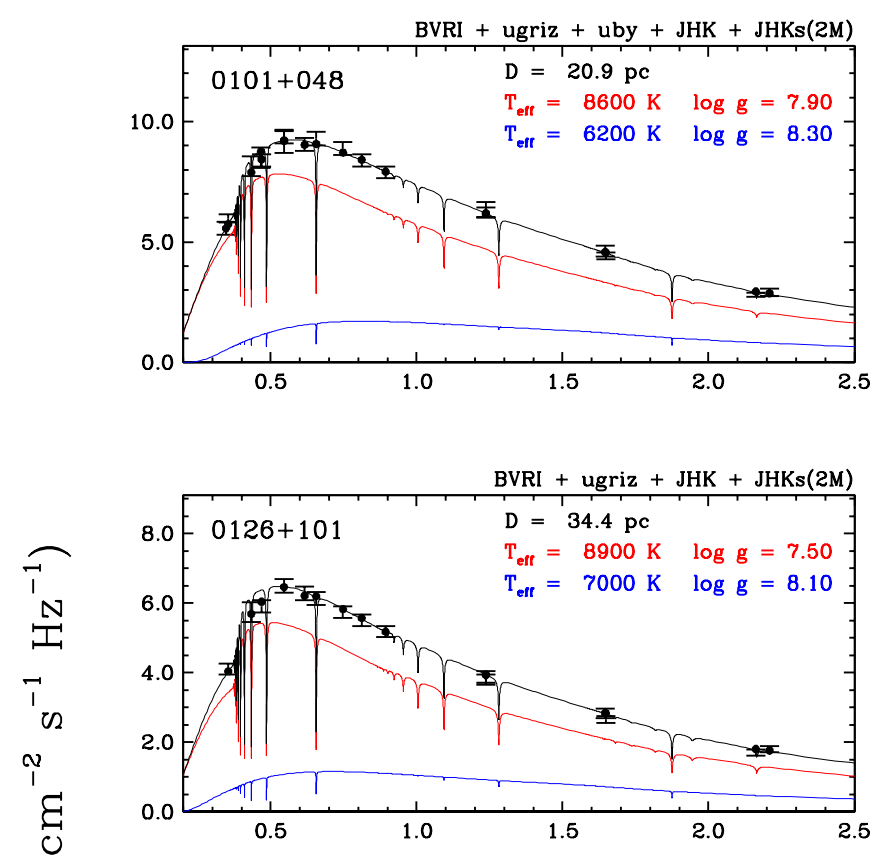

in

0
2
1
0
]

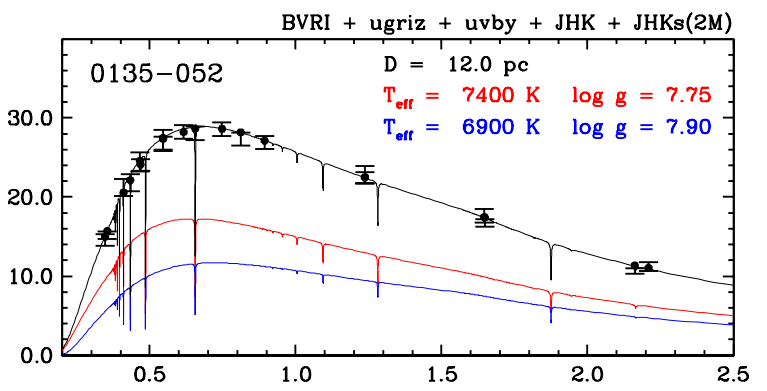

BVRI + ugri + JHK + JHKs(2M)

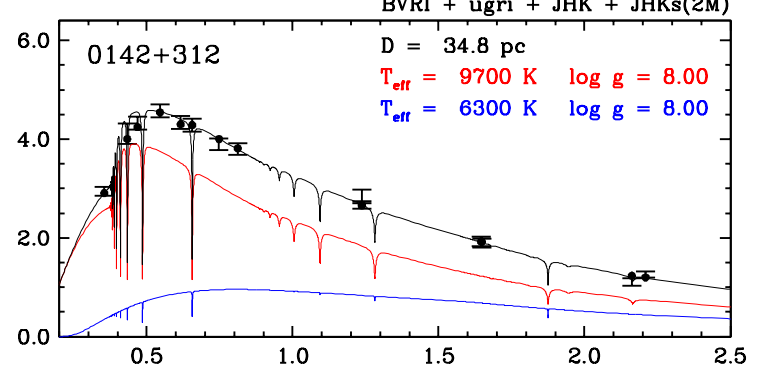

$\lambda(\mu \mathrm{m})$

Fig. 16.- Best fit to the optical spectra (left panels) and energy distributions (right panels) of the confirmed and suspected double degenerate systems identified in our analysis, assuming unresolved systems composed of two DA stars. In the right panel, the red and blue lines show the contribution of each component to the total model flux, which is displayed as the black line. 


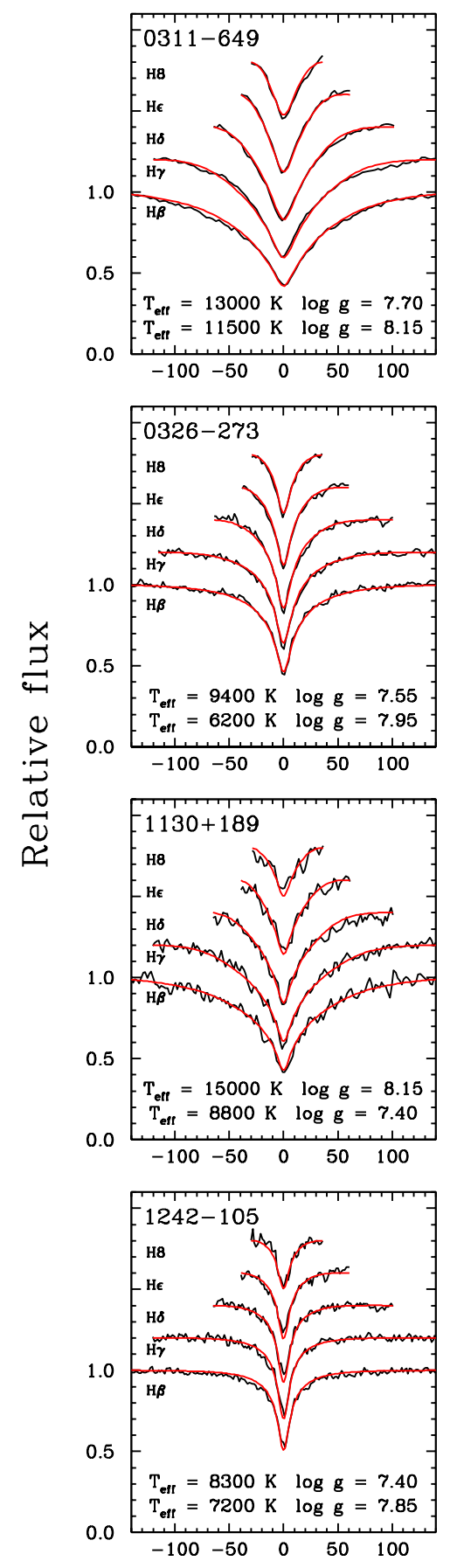

$\Delta \lambda(\AA)$
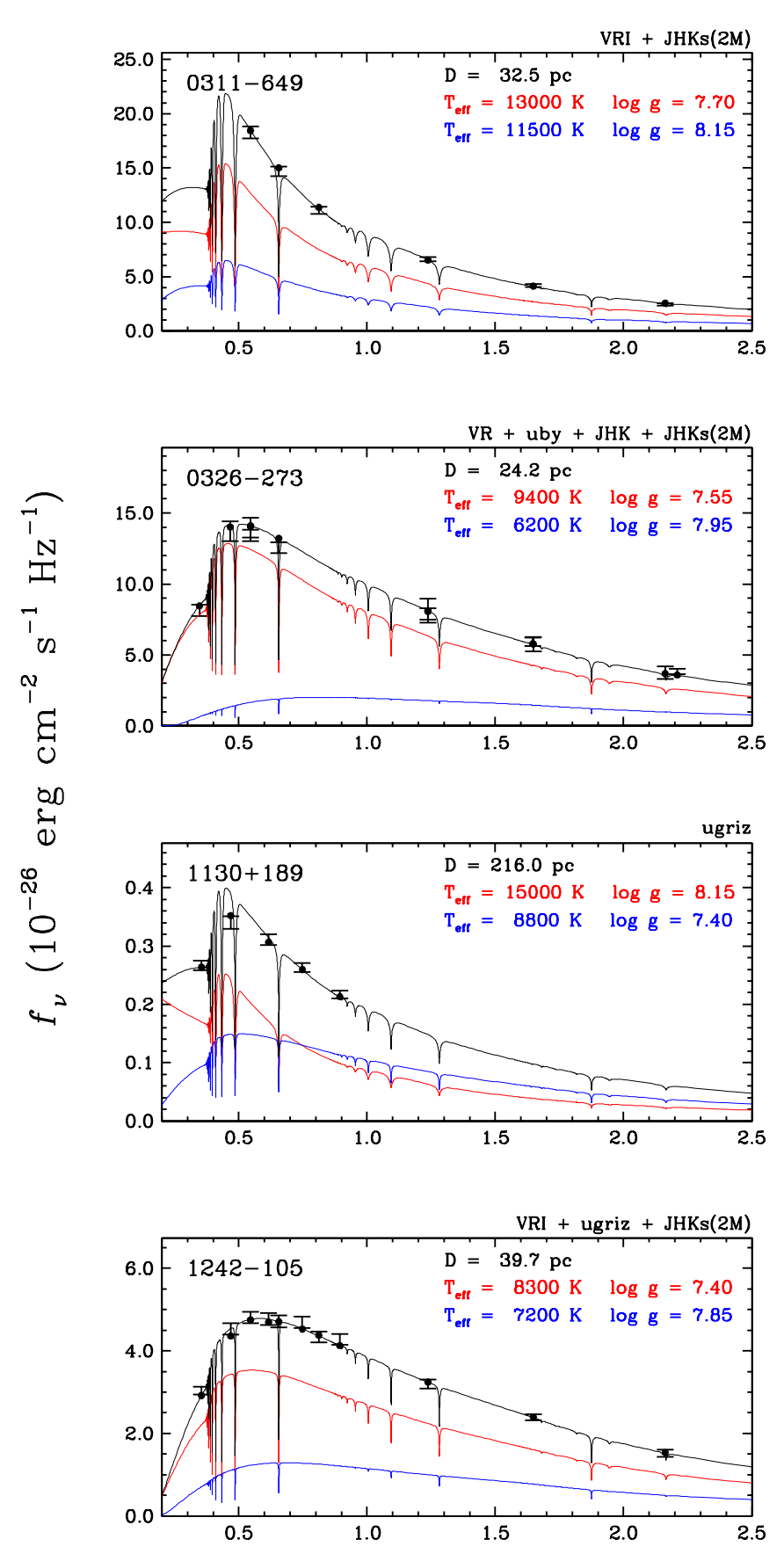

$\lambda(\mu \mathrm{m})$

Fig. 16.- (Continued) 


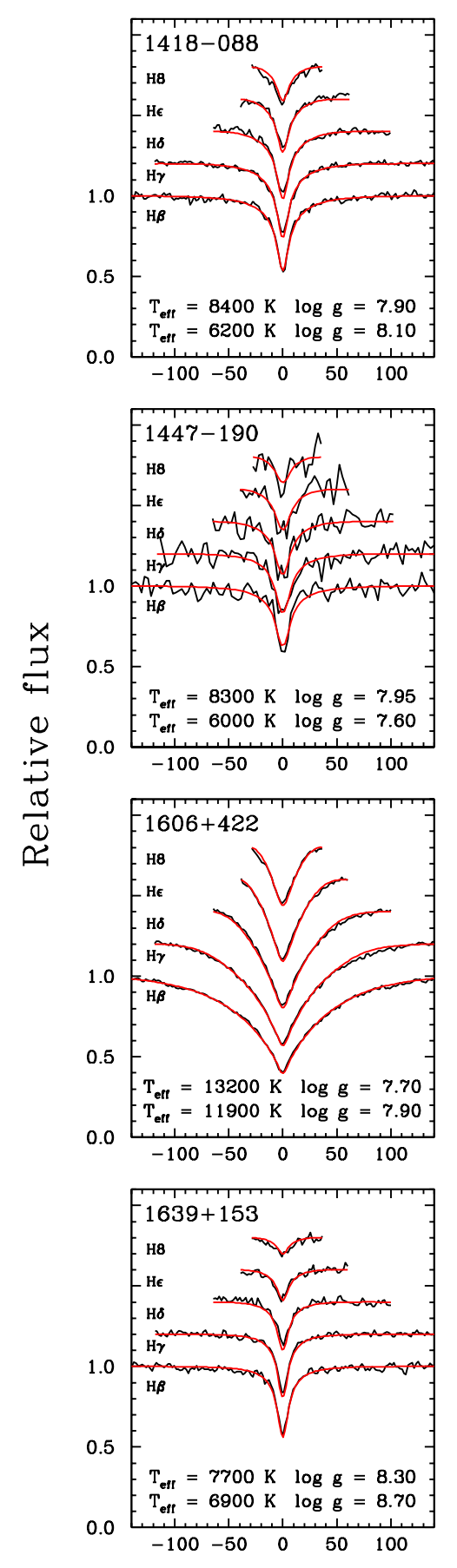

$\Delta \lambda(\AA)$
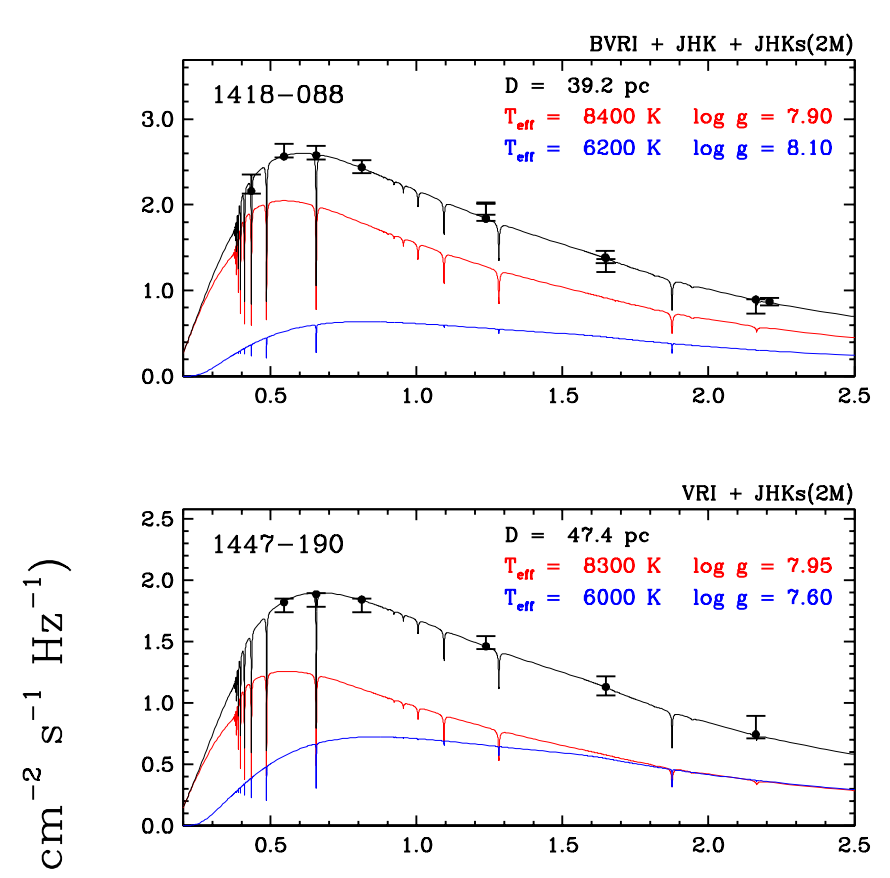

की
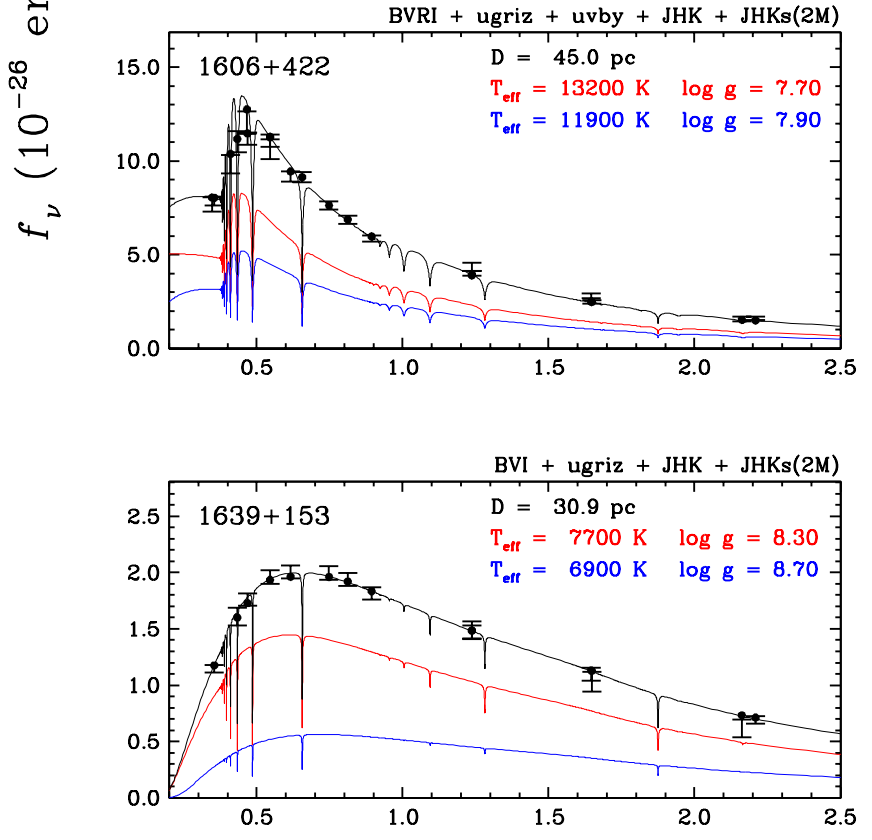

$\lambda(\mu \mathrm{m})$

Fig. 16.- (Continued) 


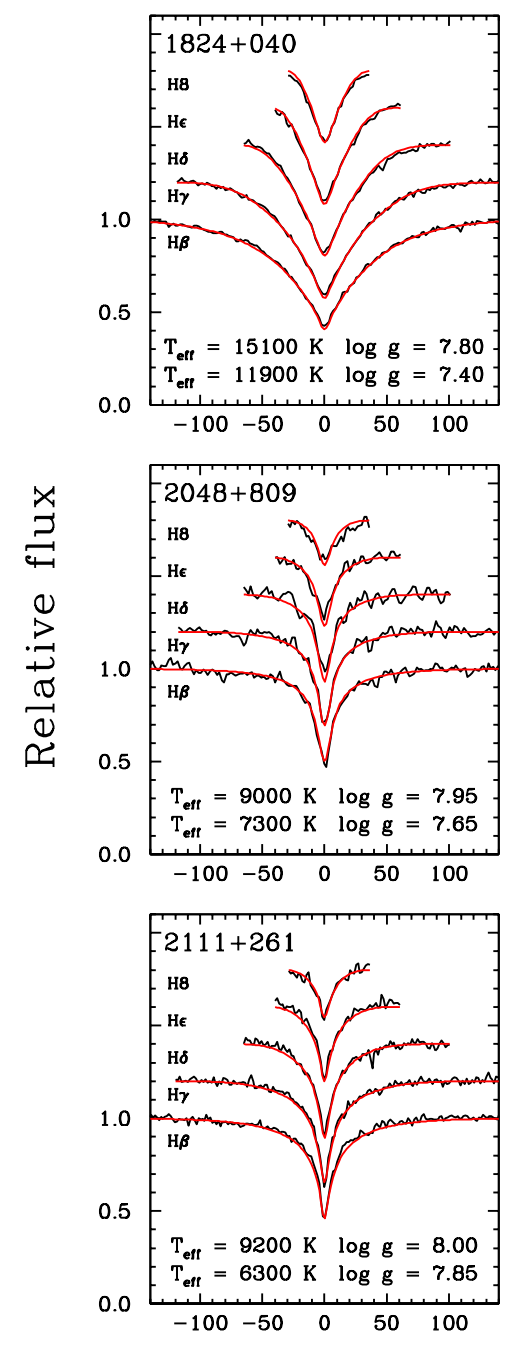

$\Delta \lambda(\AA)$

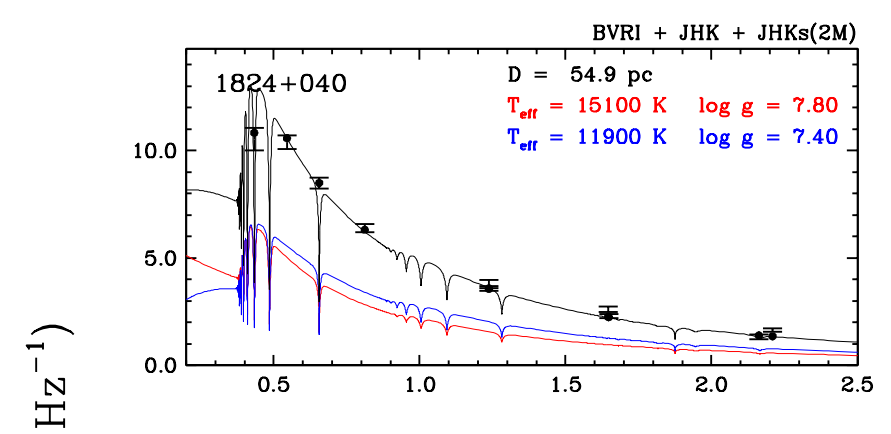

$\vec{I}_{02}$

v

हु

वै

$\stackrel{0}{\sim}$

군

4

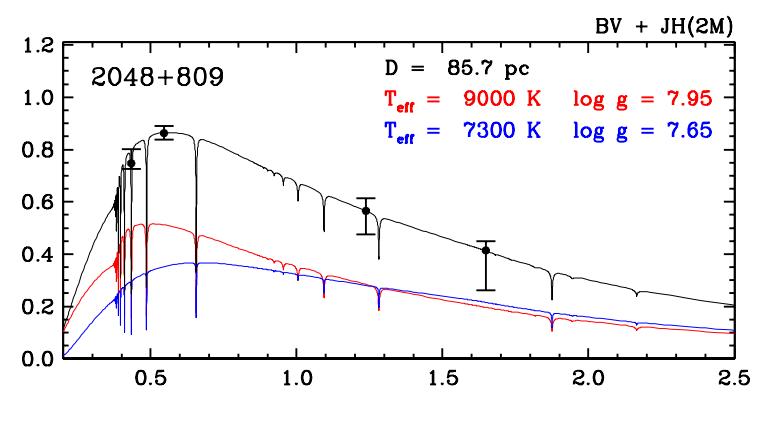

$\mathrm{BVRI}+\mathrm{uvby}+\mathrm{JHK}+\mathrm{JHKs}(2 \mathrm{M})$

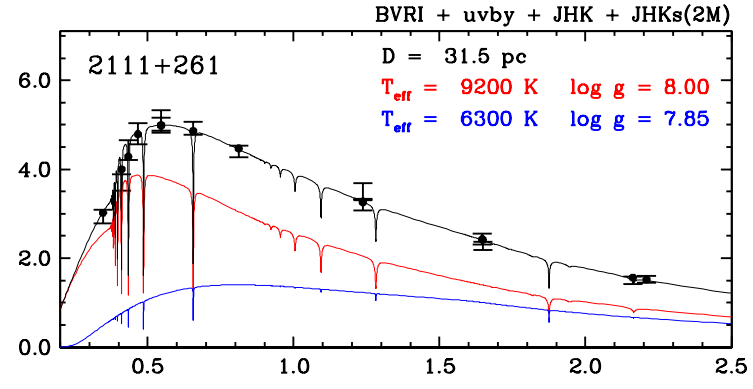

$\lambda(\mu \mathrm{m})$

Fig. 16.- (Continued) 
the luminosity of Ross 548 appears way too high, even when considered as an unresolved double degenerate binary. As a result, the photometric fit yields very low $\log g$ values, corresponding to large radii, in order to match the extreme inferred luminosity, in sharp contrast with the spectroscopic solution. There are two ways to match the high luminosity with physically acceptable $\log g$ values: either the parallax measurement is faulty and the true distance is less than $63.3 \mathrm{pc}$, or there is actually a third component in the unresolved system. Therefore, three explanations may be offered to solve the problem regarding this object: (1) Ross 548 is a single star and its distance from Earth is about 32 pc (the excess luminosity is due solely to the parallax being erroneous by a factor of 2); (2) Ross 548 is a double degenerate system and its distance from Earth is less than 63.3 pc (the excess luminosity arises from both an inaccurate parallax and the contribution of two stars); (3) Ross 548 is a triple white dwarf system and its distance from Earth is 63.3 pc (the parallax is accurate, and the excess luminosity originates from the contribution of three stars). The last possibility is admittedly unlikely but must all the same be considered. The Gaia parallax measurement for this object will hopefully help to settle the issue. We note, in the meantime, that the independent distance to Ross 548 derived by Giammichele et al. (2016) through asteroseismological means, $D=30.0 \pm 0.9 \mathrm{pc}$, favors hypothesis (1).

\subsection{Iron-Core White Dwarfs?}

An examination of the results displayed in Figures 10 and 13 shows that another class of objects occupies the location opposite to that of the double degenerate binaries in these plots, that is, above the 1:1 correspondence in the $D_{\mathrm{MR}}$ versus $D_{\pi}$ diagram, and on the left of the theoretical mass-radius relation in the $R_{\pi}$ versus $M$ diagram. One possible explanation to account for this feature is that these white dwarfs actually have a core composed of iron rather than carbon and oxygen. Indeed, Figure 13 shows that these objects fall closer to the predicted curve corresponding to Fe-core models. We explore this possibility in detail in the present section.

The most notorious case of a white dwarf that is possibly made of iron-rich material is $0644+375$ (G87-7). The Fe-core hypothesis was first suggested by Provencal et al. (1998), who obtained mass and radius values consistent with the zero-temperature Fe-core mass-radius relation of Hamada \& Salpeter (1961). Further investigations by Fontaine et al. (2007) using improved spectroscopic parameters and finite-temperature evolutionary models with different core compositions provided additional elements in favor of this scenario. Since then, there have been several developments regarding the data and models, namely, the new reduction of the Hipparcos data by van Leeuwen (2007), the upgraded Stark pro- 
files of Tremblay \& Bergeron (2009), and the new Fe-core evolutionary models described in Section 4.2 of the present study. Thus, we revisit the case of G87-7 in light of these new developments, using our standard approach for testing the mass-radius relation.

To improve the accuracy of our analysis, we first rederive the spectroscopic parameters of G87-7 by making use of the four high-quality ( $\mathrm{S} / \mathrm{N} \sim 80$ ) optical spectra available to us, as was done by Fontaine et al. (2007). The four individual fits are not displayed here but are all similar to our original fit shown in Figure 3 in terms of quality. We obtain mean values of $T_{\text {eff }}=21,996 \pm 254 \mathrm{~K}$ and $\log g=8.148 \pm 0.043$, where the uncertainties include both the internal and external errors as usual, but in the present case we adopt as the external errors the standard deviations associated with the parameters derived from the four individual fits, rather than the prescription of Liebert et al. (2005). Our improved spectroscopic parameters are consistent with our previous estimates $\left(T_{\text {eff }}=22,143 \pm 337 \mathrm{~K}, \log g=8.094 \pm 0.045\right)$, but we note that the mean $\log g$ value is slightly larger than our original value obtained from a single spectrum.

We then apply our standard procedure outlined in Section 4.1, using successively our $\mathrm{C} / \mathrm{O}$-core and Fe-core evolutionary models (still with thick hydrogen layers). The results are displayed in Figure 17 through our typical $D_{\mathrm{MR}}$ versus $D_{\pi}$ and $R_{\pi}$ versus $M$ comparisons. Unlike the $\mathrm{C} / \mathrm{O}$-core mass-radius relation, the Fe-core mass-radius relation yields an almost perfect agreement. More specifically, the difference between the distance estimates is significantly smaller if we assume an iron core $(\Delta D=0.15 \sigma)$ instead of a carbon/oxygen core $(\Delta D=1.67 \sigma)$. That being said, $\Delta D=1.67 \sigma$ is not a largely inconsistent result, and it is still plausible that G87-7 is a normal C/O-core white dwarf. However, we stress that all the physical quantities for this bright, nearby star are remarkably well-constrained: the Hipparcos parallax measurement has a small 5.6\% uncertainty, the spectroscopic parameters are obtained from four high-quality spectra, and the photometric parameters are also welldetermined since the energy distribution is modeled from magnitudes in 13 bandpasses in the optical and infrared. Although it is possible that the Hipparcos parallax suffers from a systematic error, as in the case of $1314+293$ mentioned in Section 2.2, this appears highly unlikely since the Hipparcos measurement is in good agreement with previous ground-based measurements. Thus, our analysis provides fairly strong support to the idea that G87-7 harbors an iron core, although a carbon/oxygen core cannot be ruled out.

In addition to G87-7, our sample contains 13 other white dwarfs having distance differences larger than $1.5 \sigma$ that might be explained by an iron-rich core: $0011+000(\Delta D=2.17 \sigma)$, $0842+490(\Delta D=2.93 \sigma), 0942+236 \mathrm{~A}(\Delta D=2.16 \sigma), 1105-340(\Delta D=1.64 \sigma), 1609+135$ $(\Delta D=2.49 \sigma), 1620-391(\Delta D=3.44 \sigma), 1635+137(\Delta D=1.66 \sigma), 1645+325(\Delta D=$ $1.85 \sigma), 1916-362(\Delta D=2.62 \sigma), 1936+327(\Delta D=2.22 \sigma), 1950+250(\Delta D=1.82 \sigma)$, 

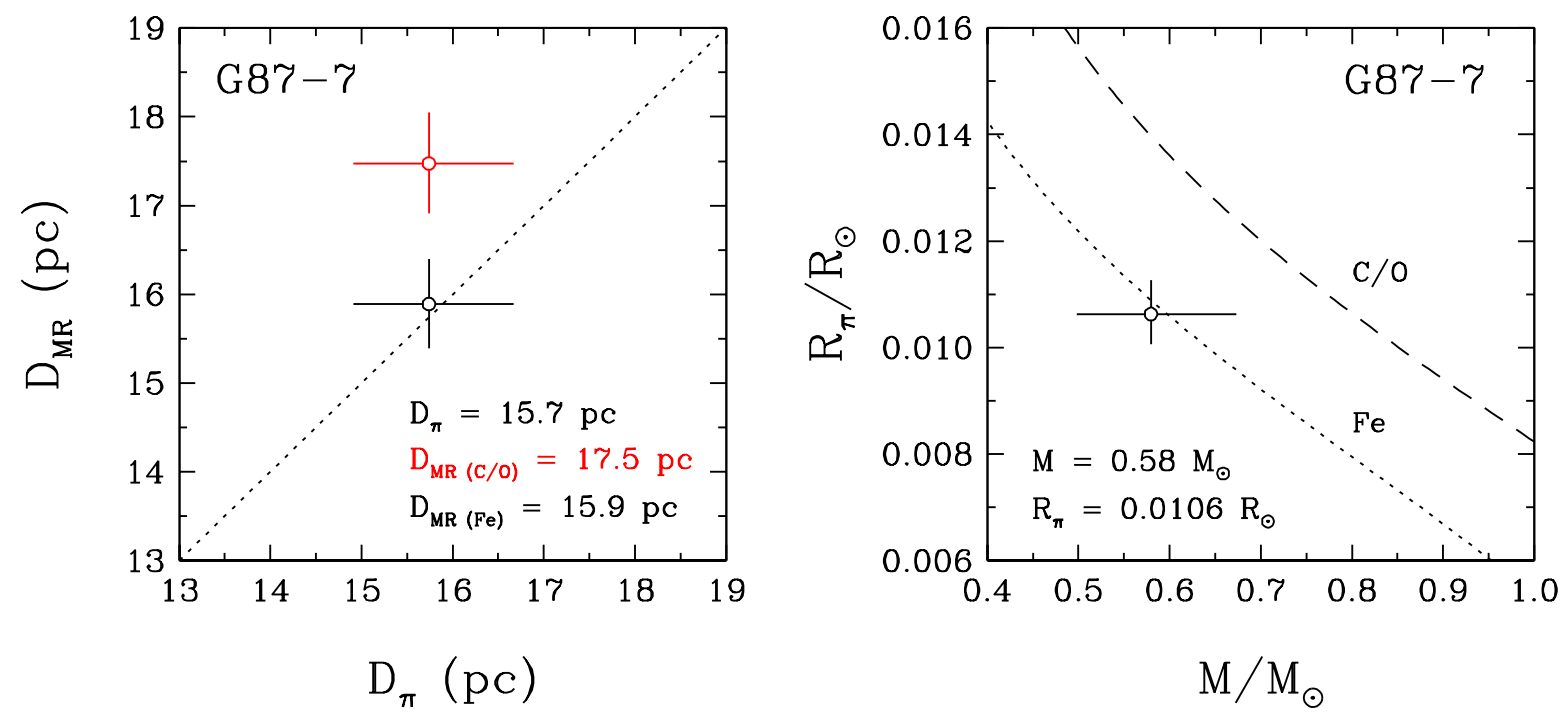

Fig. 17. - Location of $0644+375$ (G87-7) in the $D_{\mathrm{MR}}$ versus $D_{\pi}$ diagram (left panel) assuming $\mathrm{C} / \mathrm{O}$-core models (red) or Fe-core models (black). Location of the same object in the $R_{\pi}$ versus $M$ diagram (right panel) together with mass-radius relations for $\mathrm{C} / \mathrm{O}$-core (dashed line) and Fe-core (dotted line), thick hydrogen envelope models at the effective temperature of G87-7. 
2105-820 $(\Delta D=5.54 \sigma)$, and 2336-079 $(\Delta D=3.76 \sigma)$. The location of these objects in the $R_{\pi}$ versus $M$ diagram is shown in Figure 18, together with three Fe-core theoretical curves corresponding to $T_{\text {eff }}=7000,15,000$, and 25,000 K. The C/O-core mass-radius relation at $T_{\text {eff }}=15,000 \mathrm{~K}$ is also displayed as a reference. Six objects (shown in black) are consistent with the Fe-core mass-radius relation within $1 \sigma$; besides G87-7, these correspond to 1105-340, 1620-3915, 1645+325, 1936+327, and 1950+250. However, we note from Figure 18 that some of these objects are only marginally consistent with the Fe-core curve, and hence the evidence in favor of the Fe-core hypothesis is less convincing than for G87-7. Only three points clearly fall on the predicted curve for an iron core, one of which corresponds to G87-7. The two other objects are 1105-340 and 1620-391, which have precise parallax and spectroscopic $\log g$ measurements, as it is the case for G87-7. These two nearby stars are thus fairly good candidates for an iron core. The remaining objects (shown in red) are simply located too far on the left in the diagram to be considered as possible Fe-core white dwarfs, and we currently have no alternative physical explanation to account for the observed discrepancies, except in the case of 2105-820, which is discussed in detail in Section 4.6. Still, we note that $0011+000,1609+135$, and 2336-079 would agree much better with the $\mathrm{C} / \mathrm{O}$-core mass-radius relation had we not applied the $3 \mathrm{D}$ correction to the spectroscopic $\log g$ (these three stars have effective temperatures close to $\sim 10,000 \mathrm{~K}$, at which the $\log g$ correction reaches its maximum value). However, there is currently no physical justification to follow such a procedure. As argued in Section 4.3, new trigonometric parallax measurements might help to improve the situation.

The major difficulty with the Fe-core hypothesis is that there is no conventional way in stellar evolution theory to produce iron-core white dwarfs with, in particular, masses less than the average mass as shown by the candidates in Figure 18. However, we draw the attention of the reader to the interesting suggestion of Ouved et al. (2011) who argued that, under certain circumstances, Fe-rich white dwarfs with masses in the range $0.43<M / M_{\odot}<0.72$ could be produced through quark-nova explosions in low-mass X-ray binaries. The predicted mass range is particularly suggestive in view of the results depicted in Figure 18.

5 Holberg et al. (2012) find an excellent agreement with C-core models for 1620-391 (CD -38 10980 ), although their spectroscopic $\log g$ value of 8.099 is based on an old determination from Bragaglia et al. (1995), which is significantly different from ours ( $\log g=7.965)$ based on a more recent optical spectrum and model atmosphere analysis. 


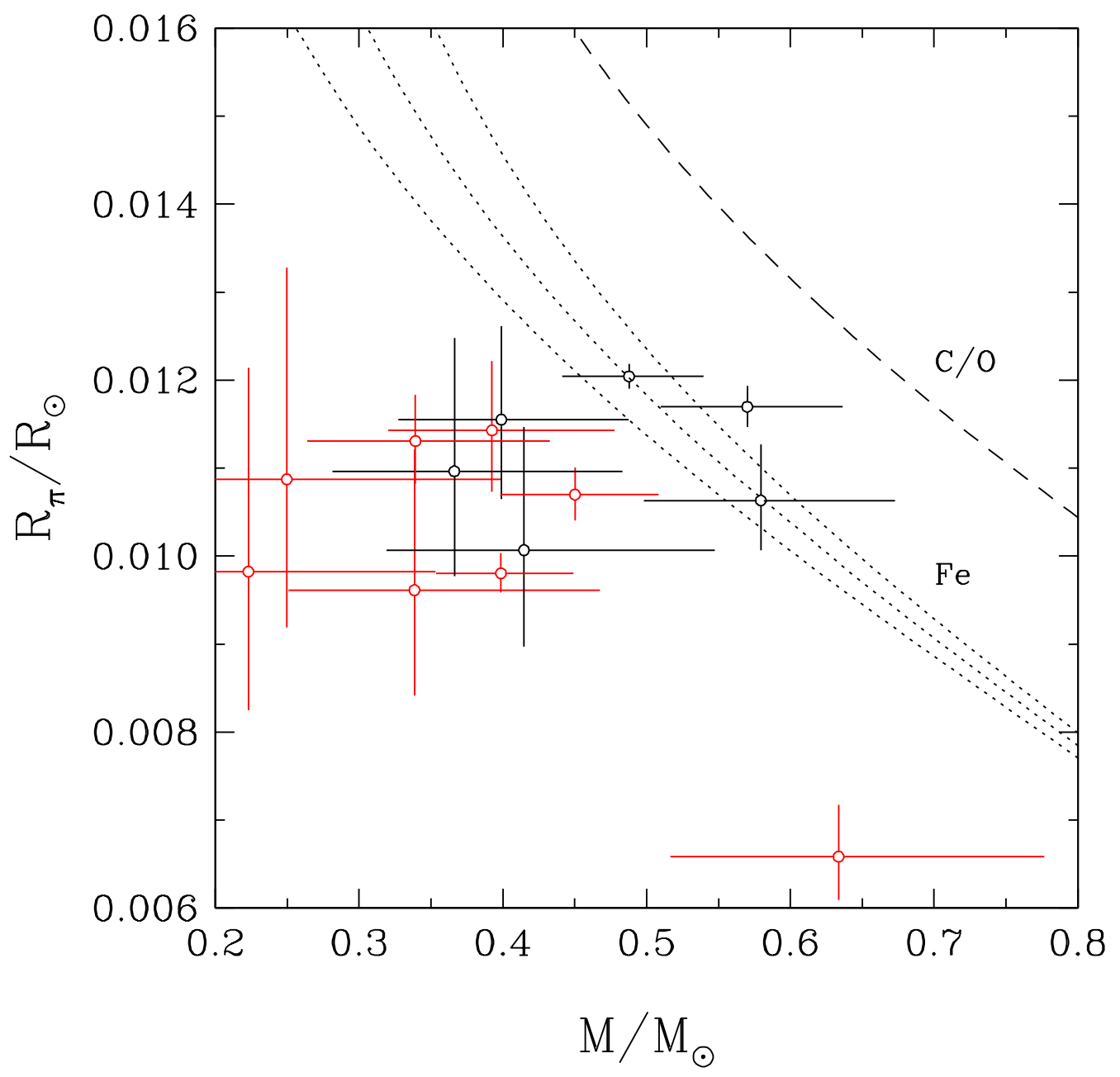

Fig. 18.- Location in the $R_{\pi}$ versus $M$ diagram of the white dwarfs in our sample that are possibly composed of an iron core. Also shown are mass-radius relations for Fe-core, thick hydrogen envelope models at $T_{\text {eff }}=7000,15,000$, and 25,000 $\mathrm{K}$ (dotted lines, from left to right), and for $\mathrm{C} / \mathrm{O}$-core, thick hydrogen envelope models at $T_{\text {eff }}=15,000 \mathrm{~K}$ (dashed line). The objects shown in red are not consistent with the Fe-core mass-radius relation within a $1 \sigma$ confidence level. 


\subsection{5-820: A Magnetic White Dwarf with a Radiative Atmosphere?}

2105-820 (L24-52) is a DA star with a very precise trigonometric parallax measurement from CTIOPI of $64.81 \pm 1.39$ mas. Also, because it is bright $(V \sim 13.6)$ and hot $\left(T_{\text {eff }} \sim 10,000 \mathrm{~K}\right)$, its photometric energy distribution and optical spectrum are both accurately measured, and the corresponding photometric and spectroscopic solutions are very well constrained. Yet, the distance inferred from the mass-radius relation, $D_{\mathrm{MR}}=19.5 \mathrm{pc}$, differs from the parallactic distance, $D_{\pi}=15.4 \mathrm{pc}$, by more than $5.5 \sigma$, and its location in the $R_{\pi}$ versus $M$ diagram is largely inconsistent with the mass-radius relations obtained from $\mathrm{C} / \mathrm{O}$-core models, or even from Fe-core models. These results are summarized in Figure 19 by the red symbols. A similar discrepancy, albeit somewhat less significant, is observed between the spectroscopic temperature, $T_{\text {spec }}=10,369 \mathrm{~K}$, and the photometric temperature, $T_{\text {phot }}=9921 \mathrm{~K}$, which corresponds to $\Delta T_{\text {eff }}=1.94 \sigma$. Moreover, since 2105-820 lies on the left-hand side of the mass-radius relation, it cannot be interpreted as an unresolved degenerate binary.

Landstreet et al. (2012) reported the discovery of an apparently constant longitudinal magnetic field of $\sim 9.5 \mathrm{kG}$ in 2105-820, consistent with a simple dipolar morphology with a magnetic axis almost parallel to the rotation axis, and a polar strength of $\sim 56$ $\mathrm{kG}$. Interestingly enough, recent studies have shown that magnetic fields of only a few $\mathrm{kG}$ can suppress convective energy transport in white dwarf atmospheres (Valyavin et al. 2014; Tremblay et al. 2015). With this idea in mind, we have reanalyzed all photometric and spectroscopic data using model atmospheres in which convective energy transport has been completely suppressed (Lecavalier-Hurtubise \& Bergeron 2017). Lecavalier-Hurtubise \& Bergeron have shown in particular that even though photometric temperatures are only marginally affected by the use of purely radiative or convective model atmospheres, the spectroscopic temperatures measured from both sets of models can differ by more than $\sim 2000 \mathrm{~K}$.

Our results of this experiment for 2105-820 are displayed in Figure 19 by the black symbols. With the use of purely radiative model atmospheres, the distances are now in perfect agreement $(\Delta D=0.20 \sigma)$ and its location in the $R_{\pi}$ versus $M$ diagram is also entirely consistent with the mass-radius relations obtained from $\mathrm{C} / \mathrm{O}$-core models. Also, the spectroscopic temperature obtained from purely radiative models, $T_{\text {spec }}=9797 \mathrm{~K}$, is now in much better agreement with the corresponding photometric temperature, $T_{\text {phot }}=9957 \mathrm{~K}$. These calculations strongly suggest that the correct interpretation for the discrepant results observed in Figure 19 is that 2105-820 does possess a purely radiative atmosphere, and that convective energy transport has been impeded by the $\sim 50 \mathrm{kG}$ polar magnetic field present in this star, in agreement with the calculations of Tremblay et al. (2015).

Four other white dwarfs harboring a weak magnetic field are also included in our 

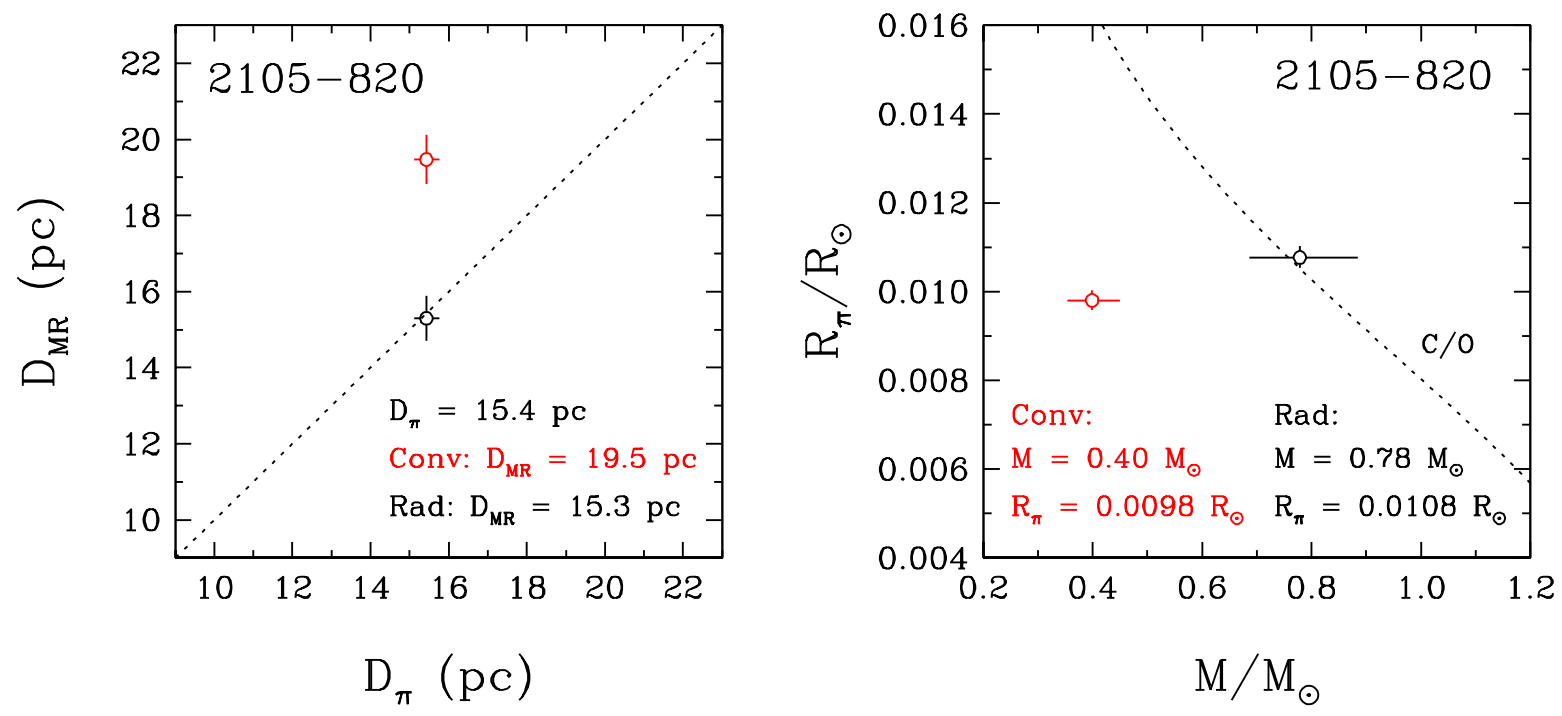

Fig. 19. - Location of $2105-820$ in the $D_{\mathrm{MR}}$ versus $D_{\pi}$ diagram (left panel) and in the $R_{\pi}$ versus $M$ diagram (right panel) using convective (red) or radiative (black) model atmospheres. 
sample: $0257+080$ (Koester et al. 2009), 1953-011 (Koester et al. 1998, 2009), 2047+372 (Landstreet et al. 2016), and 2359-434 (Landstreet et al. 2012). Contrary to the case of 2105-820, all four objects are consistent with the $\mathrm{C} / \mathrm{O}$-core mass-radius relations within $1 \sigma$ using convective model atmospheres. However, $2047+372$ is a rather hot DA white dwarf $\left(T_{\text {eff }} \sim 14,600 \mathrm{~K}\right)$ and convection in this star is negligible, while $0257+080(6620 \mathrm{~K})$, 1953-011 (7770 K), and 2359-434 (8510 K) are significantly cooler than 2105-820. Since convective energy transport is more important at lower effective temperatures, our results suggest that it may become increasingly more difficult for a weak magnetic field to suppress convection in cooler white dwarfs.

\subsection{Cool White Dwarfs with Mixed He/H Atmospheres?}

At effective temperatures below $T_{\text {eff }} \sim 10,000 \mathrm{~K}$, it is possible to hide large amounts of helium in a DA white dwarf since helium becomes spectroscopically invisible. Bergeron et al. (1991, see their Figure 16) demonstrated that a DA star with an atmosphere enriched with helium would appear exactly like a pure hydrogen white dwarf but with an apparent higher $\log g$ value, since both atmospheric parameters have the similar effect of increasing the atmospheric pressure (see also Figure 2 of Tremblay et al. 2010). Such helium enrichment most likely resulting from convective mixing - has been one of the solutions proposed to explain the longstanding high-log g problem observed in all spectroscopic analyses of cool DA stars $\left(T_{\text {eff }} \lesssim 13,000 \mathrm{~K}\right.$; also illustrated in Figure 12). However, Tremblav et al. (2010) ruled out this scenario by determining the helium abundances in several cool DA white dwarfs using high-resolution spectra from the Keck 10-m telescope. In all cases where helium could have been detected, no helium lines were observed. Tremblay et al. also explored alternative possibilities to account for this high-log $g$ problem, and finally concluded that convective energy transport treated within the mixing-length formalism was the most likely origin of this problem, an interpretation later confirmed by the 3D hydrodynamical calculations of Tremblay et al. (2011b).

Despite these negative results, we know that helium-rich DA stars with strong Balmer lines do exist, $1729+371$ (GD 362) being the best example at $T_{\text {eff }}=10,540 \mathrm{~K}, \log g=8.24$, and $N(\mathrm{He}) / \mathrm{N}(\mathrm{H}) \sim 15$ (Zuckerman et al. 2007). The atmosphere of GD 362 is thus actually dominated by helium. This DABZ star is also one of the most heavily polluted white dwarf known, but at medium resolution, the very weak He I $\lambda 5877$ absorption feature is undetectable. In the case of GD 362, hydrogen and all heavy elements have most likely been accreted from a large asteroid, or asteroids, with composition similar to the Earth-Moon system. As the white dwarf evolves, all the heavy elements will have diffused at the bottom 
of the convection zone, but hydrogen will remain thoroughly mixed within this convection zone, and the star will have evolved into a helium-dominated DA star. Thus, we cannot exclude that such objects exist in our sample, although they may be rare.

The effect of using mixed $\mathrm{H} / \mathrm{He}$ atmospheres to analyze DA stars is nicely illustrated in Figure 2 of Tremblay et al. (2010) in the case of 1655+215 (LHS 3254), also included in our sample. Their figure shows that a fit to the photometric energy distribution, constrained by the measured trigonometric parallax, yields a value of $\log g=7.84$ (using the mass-radius relation) when analyzed with pure hydrogen models, in sharp contrast with the spectroscopic value of $\log g=8.27$. It is also shown that these two surface gravities can be reconciled if model atmospheres with $N(\mathrm{He}) / \mathrm{N}(\mathrm{H})=1$ are used instead (note that the 3D hydrodynamical $\log g$ corrections were not applied in the Tremblay et al. analysis, and the amount of helium required will be lower if 3D corrections are taken into account). A further examination of Figure 2 of Tremblay et al. also reveals that the photometric method yields $\log g$ values (i.e., radius measurements) that are completely independent of the assumed atmospheric composition. Similarly, the photometric and spectroscopic temperatures are not affected either. Hence the net effect of using mixed $\mathrm{H} / \mathrm{He}$ model atmospheres on our own analysis of cool DA stars is to move an object horizontally to the left in the mass-radius plot displayed in Figure 13 (when helium-rich models are used, the measured radius remains unaffected, but the inferred mass is decreased along the $\mathrm{x}$-axis because of the resulting lower spectroscopic $\log g$ value).

With these conclusions in mind, we thus reexamined all the cool $\left(T_{\text {eff }} \lesssim 12,000 \mathrm{~K}\right)$ DA stars located on the right hand side of the $\mathrm{C} / \mathrm{O}$-core mass-radius relation in Figure 13. excluding of course the unresolved binaries, or binary candidates, mostly located in the upper right corner of this figure. For each of these stars, it is thus possible to adjust the photospheric helium abundance until the object moves (left) on top of the C/O-core massradius relation for the appropriate temperature. For instance, a helium abundance between $N(\mathrm{He}) / \mathrm{N}(\mathrm{H}) \sim 0.1$ and 1 would bring 1655+215 (see online figures) precisely on top of the $\mathrm{C} / \mathrm{O}$-core mass-radius relation. We must emphasize, however, that the distance difference for $1655+215$ using pure hydrogen models is only a $1.42 \sigma$ result, so the proposed helium-rich solution may not be significant. In some cases, the stellar mass inferred from helium-rich models is uncomfortably low. For instance, $0518+333$ is so far to the right of the mass-radius relation that the amount of helium required would bring the mass below $0.4 M_{\odot}$. However, as discussed in Section 4.4, the trigonometric parallax for this object is most likely unreliable.

All in all, we have identified about two dozen cool DA stars in our sample for which a modest helium enrichment, around $N(\mathrm{He}) / \mathrm{N}(\mathrm{H}) \sim 0.1$, would improve the agreement with the mass-radius relation. The best candidates that require an abundance close to 
$N(\mathrm{He}) / \mathrm{N}(\mathrm{H}) \sim 0.1$ are $0148+641,0250-007,2133-335$, and 2159-754 (with distance discrepancies of $1.79,2.15,1.95$, and $1.61 \sigma$, respectively). We thus conclude that there is no compelling evidence in our sample for a significant population of helium-rich DA stars, at least not at the level observed in GD 362.

\subsection{Validity of the Mass-Radius Relation}

The evidence presented in the last few sections shows that several discrepant results can be explained by various physical reasons. This evidence is particularly solid in the cases of the confirmed and suspected double degenerate binaries, and the peculiar magnetic white dwarf 2105-820. As such, the failure of the mass-radius relation to accurately predict the measured parameters of these objects is probably only apparent. Therefore, it is appropriate

to review our test of the mass-radius relation with these considerations in mind. More specifically, we exclude in the following the 15 double degenerate systems, and we assume that $2105-820$ has a purely radiative atmosphere.

Figure 20 displays the distribution of the absolute differences between the two distance estimates $D_{\mathrm{MR}}$ and $D_{\pi}$, expressed in units of $\sigma$, under these assumptions. The initial distribution of Figure 11 is also shown as a reference. Among the 143 objects (the 158 objects in our reliable subsample minus the 15 double degenerate binaries), the two distances are now within the $1 \sigma$ confidence level for $73 \%$ of the stars, and within $2 \sigma$ for $92 \%$ of the stars. Hence, the distribution now approaches the expected Gaussian distribution much more closely. Also, the number of white dwarfs showing large inconsistencies $(\Delta D \geq 3 \sigma)$ has dropped to only 3 (these correspond to 0133-116, 1620-391, and 2336-079, which were already discussed at length in Sections 4.4 and 4.5). Thus, there is no evidence for a significant statistical deviation from the theoretical mass-radius relation, and we can state that our empirical analysis provides strong support to the current theory of stellar degeneracy.

Furthermore, we can now obtain a mass distribution that is independent of the massradius relation (from the mass values $M$ corresponding to the x-axis of our $R_{\pi}$ versus $M$ diagram). To our knowledge, such a mass distribution is presented for the first time. This mass distribution is shown as the hatched black histogram in Figure 7 , together with the spectroscopic and photometric mass distributions discussed previously. The mean mass, $\langle M\rangle=0.666 M_{\odot}$, is comparable to those of the two other distributions, albeit slightly higher. Also, the distribution is somewhat flatter, which results in a larger standard deviation, $\sigma_{M}=0.213 M_{\odot}$. A careful examination of our results reveals that this larger standard deviation can be explained in part by the presence of extended low-mass and high-mass tails, which contain respectively 13 and 14 white dwarfs for which $\Delta D>1 \sigma$. For instance, 


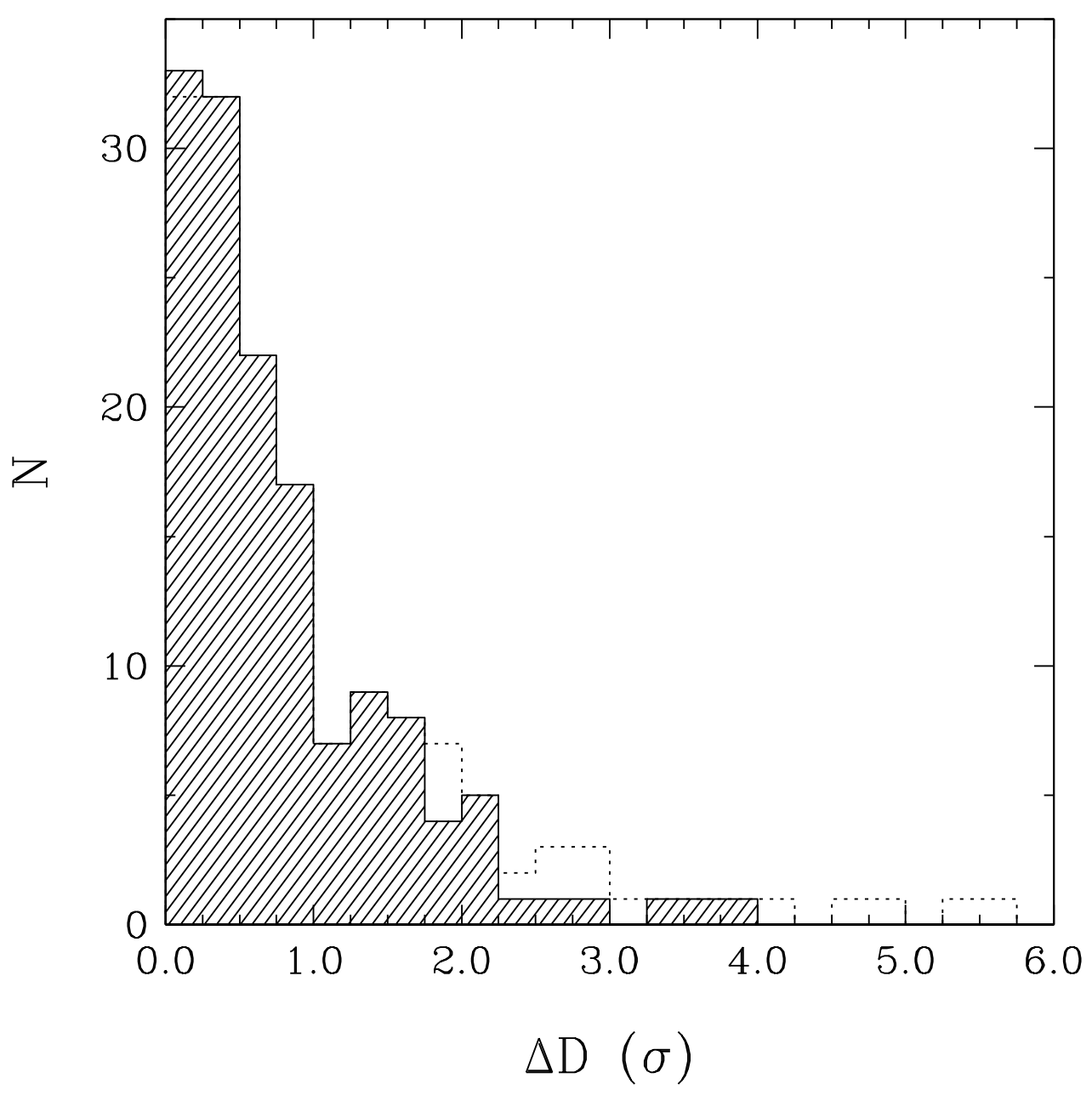

Fig. 20.- Distribution of the (absolute) differences between the distances $D_{\mathrm{MR}}$ and $D_{\pi}$, measured in units of $\sigma$, where $\sigma^{2} \equiv \sigma_{D_{\mathrm{MR}}}^{2}+\sigma_{D_{\pi}}^{2}$, for all white dwarfs in our sample with reliable trigonometric parallax and spectroscopic $\log g$ measurements, after exclusion of the 15 double degenerate candidates, and under the assumption that 2105-820 has a purely radiative atmosphere. The initial distribution of Figure 11 is shown as the dotted line for comparison. 
the suspiciously high mass of $M=1.34 M_{\odot}$ obtained for $1509+322$ must be considered doubtful since the parallax measurement for this object has a large $19.2 \%$ uncertainty, close to our confidence limit of $20 \%$. Similarly, we obtained an aberrant mass of $M=2.30 M_{\odot}$ for 0133-116, which was not included in the calculation of the mean value and standard deviation of the distribution, for the obvious reason that this mass is undoubtedly largely overestimated, as discussed in Section 4.4.

We finally summarize in Table 1 the results of our analysis for the 158 white dwarfs (or white dwarf systems) with reliable data, where we give for each object the WD number, name, and spectral type, the parallactic distance $\left(D_{\pi}\right)$, the effective temperature $\left(T_{\text {eff }}\right)$ and the method used to determine this temperature, the spectroscopic surface gravity determination $(\log g)$, the radius $\left(R_{\pi}\right)$ derived from the photometric technique, and the mass $(M)$ obtained by combining the values of $\log g$ and $R_{\pi}$. We also give, as a quantitative measure of consistency with the $\mathrm{C} / \mathrm{O}$-core mass-radius relation, the difference between the distances $D_{\pi}$ and $D_{\mathrm{MR}}$, in units of $\sigma$. We want to emphasize that since we have established the validity of the mass-radius relation, the physical parameters for objects with large $\Delta D$ values (i.e., objects that deviate from the predictions of the mass-radius relation) should be regarded with caution. In particular, note that the parameters for the double degenerate binaries (or binary candidates) are given here under the assumption of a single star, and the reader should refer to Figure [16 to obtain the deconvolved parameters. It is probable that at least some of the remaining discrepant cases will be resolved by improved astrometric, spectroscopic and/or photometric data. However, it is also possible that some of these white dwarfs have yet unknown specific characteristics that still await a correct interpretation.

\section{CONCLUSION}

We performed a detailed model atmosphere analysis of 219 DA and DB white dwarfs with measured trigonometric parallaxes, using both the spectroscopic and photometric techniques. The physical parameters essential to the characterization of white dwarf stars were obtained in a homogeneous way from fits to optical spectra and to energy distributions. After focusing our attention to a subsample of 158 objects with reliable surface gravity determinations and precise parallax measurements, we showed that the effective temperatures and masses derived from spectroscopy and photometry are generally in good agreement.

The physical parameters were then compared to those predicted by the theoretical mass-radius relation for white dwarfs. Since $92 \%$ of the stars in our reliable subsample were found to be consistent with the mass-radius relation within a $2 \sigma$ confidence level, we can confidently assert that the theory of degenerate stars rests on solid empirical grounds. We 
therefore reach the same conclusion as Holberg et al. (2012), Tremblay et al. (2017), and Parsons et al. (2017), but for a much larger sample of white dwarfs, thus greatly improving the observational constraints on this widely used theoretical model. However, we are on the verge of an even more important improvement of our ability to investigate the degenerate mass-radius relation. Indeed, the upcoming data releases of the Gaia mission will significantly increase the number and precision of white dwarf parallax measurements. However, high-quality spectroscopy and photometry will be needed as well to obtain accurate physical quantities, and hence to advance work in this field of research.

It was also demonstrated that the systematic approach used to study the validity of the mass-radius relation offers the indirect advantage of unveiling physical peculiarities of individual white dwarfs, which we fully exploited. Indeed, we presented convincing evidence that 15 objects in our reliable subsample are most certainly unresolved double degenerate binaries. Based on these results, the proportion of unresolved double degenerate systems among the white dwarf population can be estimated to be $\sim 10 \%$. We pushed our analysis further by showing that the spectroscopic and photometric observations for all these objects can be fitted by composite models, and atmospheric parameters were derived for both components in these binary systems separately. This opens up a new window to study the physical properties of white dwarfs in double degenerate systems on a large scale. The solutions presented here could eventually be confirmed by comparisons with high-resolution spectroscopy resolving both components.

While the current level of precision on the white dwarf parameters does not allow for a significant determination of the hydrogen envelope thickness, it is nevertheless possible to probe the internal composition. Our findings indicate that there might be some exceptions to the widely accepted assumption that stellar evolution produces white dwarfs having a core composed mostly of carbon and oxygen. More specifically, three objects in our sample fall directly on the theoretical mass-radius relation for an iron core. The most compelling case is that of $0644+375$ (G87-7), for which the parameters are all firmly pinned down by high-quality observations, and the possibility of an iron core for this object must be considered seriously. This interpretation definitely challenges the current theories of white dwarf formation.

This work was supported in part by the NSERC Canada and by the Fund FRQ-NT (Québec). 


\section{REFERENCES}

Barstow, M. A., Bond, H. E., Burleigh, M. R., Casewell, S. L., Farihi, J., Holberg, J. B., \& Hubeny, I. 2015, in Astronomical Society of the Pacific Conference Series, Vol. 493, 19th European Workshop on White Dwarfs, ed. P. Dufour, P. Bergeron, \& G. Fontaine, 307

Barstow, M. A., Bond, H. E., Holberg, J. B., Burleigh, M. R., Hubeny, I., \& Koester, D. 2005, MNRAS, 362, 1134

Bergeron, P., Leggett, S. K., \& Harris, H. C. 2009, Journal of Physics Conference Series, 172,012062

Bergeron, P., Leggett, S. K., \& Ruiz, M. T. 2001, ApJS, 133, 413

Bergeron, P., Liebert, J., \& Fulbright, M. S. 1995a, ApJ, 444, 810

Bergeron, P., Rolland, B., Limoges, M.-M., Giammichele, N., Séguin-Charbonneau, L., \& Green, E. M. 2015, in Astronomical Society of the Pacific Conference Series, Vol. 493, 19th European Workshop on White Dwarfs, ed. P. Dufour, P. Bergeron, \& G. Fontaine, 33

Bergeron, P., Ruiz, M. T., \& Leggett, S. K. 1997, ApJS, 108, 339

Bergeron, P., Saffer, R. A., \& Liebert, J. 1992a, ApJ, 394, 228

Bergeron, P., Wesemael, F., Dufour, P., Beauchamp, A., Hunter, C., Saffer, R. A., Gianninas, A., Ruiz, M. T., Limoges, M.-M., Dufour, P., Fontaine, G., \& Liebert, J. 2011, ApJ, 737,28

Bergeron, P., Wesemael, F., \& Fontaine, G. 1991, ApJ, 367, 253

-. 1992b, ApJ, 387, 288

Bergeron, P., Wesemael, F., Fontaine, G., \& Liebert, J. 1989, ApJ, 345, L91

Bergeron, P., Wesemael, F., Lamontagne, R., Fontaine, G., Saffer, R. A., \& Allard, N. F. 1995b, ApJ, 449, 258

Bond, H. E., Schaefer, G. H., Gilliland, R. L., Holberg, J. B., Mason, B. D., Lindenblad, I. W., Seitz-McLeese, M., Arnett, W. D., Demarque, P., Spada, F., Young, P. A., Barstow, M. A., Burleigh, M. R., \& Gudehus, D. 2017, ApJ, 840, 70

Bragaglia, A., Renzini, A., \& Bergeron, P. 1995, ApJ, 443, 735 
Cutri, R. M., Skrutskie, M. F., van Dyk, S., Beichman, C. A., Carpenter, J. M., Chester, T., Cambresy, L., Evans, T., Fowler, J., Gizis, J., Howard, E., Huchra, J., Jarrett, T., Kopan, E. L., Kirkpatrick, J. D., Light, R. M., Marsh, K. A., McCallon, H., Schneider, S., Stiening, R., Sykes, M., Weinberg, M., Wheaton, W. A., Wheelock, S., \& Zacarias, N. 2003, 2MASS All Sky Catalog of point sources.

Dahn, C. C., Bergeron, P., Liebert, J., Harris, H. C., Canzian, B., Leggett, S. K., \& Boudreault, S. 2004, ApJ, 605, 400

Dahn, C. C., Harrington, R. S., Kallarakal, V. V., Guetter, H. H., Luginbuhl, C. B., Riepe, B. Y., Walker, R. L., Pier, J. R., Vrba, F. J., Monet, D. G., \& Ables, H. D. 1988, AJ, 95,237

Dahn, C. C., Harrington, R. S., Riepe, B. Y., Christy, J. W., Guetter, H. H., Kallarakal, V. V., Miranian, M., Walker, R. L., Vrba, F. J., Hewitt, A. V., Durham, W. S., \& Ables, H. D. 1982, AJ, 87, 419

Debes, J. H., Kilic, M., Tremblay, P.-E., López-Morales, M., Anglada-Escude, G., Napiwotzki, R., Osip, D., \& Weinberger, A. 2015, AJ, 149, 176

Ducourant, C., Teixeira, R., Hambly, N. C., Oppenheimer, B. R., Hawkins, M. R. S., Rapaport, M., Modolo, J., \& Lecampion, J. F. 2007, A\&A, 470, 387

Dufour, P., Bergeron, P., \& Fontaine, G. 2005, ApJ, 627, 404

Dufour, P., Bergeron, P., Liebert, J., Harris, H. C., Knapp, G. R., Anderson, S. F., Hall, P. B., Strauss, M. A., Collinge, M. J., \& Edwards, M. C. 2007, ApJ, 663, 1291

Falcon, R. E., Winget, D. E., Montgomery, M. H., \& Williams, K. A. 2010, ApJ, 712, 585

-. 2012, ApJ, 757, 116

Farihi, J. 2009, MNRAS, 398, 2091

Farihi, J., Becklin, E. E., \& Zuckerman, B. 2005, ApJS, 161, 394

Finley, D. S., Koester, D., \& Basri, G. 1997, ApJ, 488, 375

Fontaine, G., Bergeron, P., \& Brassard, P. 2007, in Astronomical Society of the Pacific Conference Series, Vol. 372, 15th European Workshop on White Dwarfs, ed. R. Napiwotzki \& M. R. Burleigh, 13

Fontaine, G., Bergeron, P., Lacombe, P., Lamontagne, R., \& Talon, A. 1985, AJ, 90, 1094 
Fontaine, G., Brassard, P., \& Bergeron, P. 2001, PASP, 113, 409

Gaia Collaboration. 2016, VizieR Online Data Catalog, 1337

Genest-Beaulieu, C. \& Bergeron, P. 2014, ApJ, 796, 128

Giammichele, N., Bergeron, P., \& Dufour, P. 2012, ApJS, 199, 29

Giammichele, N., Fontaine, G., Brassard, P., \& Charpinet, S. 2016, ApJS, 223, 10

Gianninas, A., Bergeron, P., \& Ruiz, M. T. 2011, ApJ, 743, 138

Graham, J. A. 1972, AJ, 77, 144

Greenstein, J. L. 1976, AJ, 81, 323

Hamada, T. \& Salpeter, E. E. 1961, ApJ, 134, 683

Harrington, R. S. \& Dahn, C. C. 1980, AJ, 85, 454

Harrington, R. S., Kallarakal, V. V., Christy, J. W., Dahn, C. C., Riepe, B. Y., Guetter, H. H., Ables, H. D., Hewitt, A. V., Vrba, F. J., \& Walker, R. L. 1985, AJ, 90, 123

Harris, H. C., Dahn, C. C., Dupuy, T. J., Canzian, B., Guetter, H. H., Hartkopf, W. I., Ireland, M. J., Leggett, S. K., Levine, S. E., Liu, M. C., Luginbuhl, C. B., Monet, A. K. B., Stone, R. C., Subasavage, J. P., Tilleman, T., \& Walker, R. L. 2013, ApJ, 779,21

Holberg, J. B. \& Bergeron, P. 2006, AJ, 132, 1221

Holberg, J. B., Bergeron, P., \& Gianninas, A. 2008, AJ, 135, 1239

Holberg, J. B., Oswalt, T. D., \& Barstow, M. A. 2012, AJ, 143, 68

Kepler, S. O., Pelisoli, I., Koester, D., Ourique, G., Kleinman, S. J., Romero, A. D., Nitta, A., Eisenstein, D. J., Costa, J. E. S., Külebi, B., Jordan, S., Dufour, P., Giommi, P., \& Rebassa-Mansergas, A. 2015, MNRAS, 446, 4078

Kidder, K. M., Holberg, J. B., \& Mason, P. A. 1991, AJ, 101, 579

Koen, C., Kilkenny, D., van Wyk, F., \& Marang, F. 2010, MNRAS, 403, 1949

Koester, D. 1987, ApJ, 322, 852

Koester, D., Dreizler, S., Weidemann, V., \& Allard, N. F. 1998, A\&A, 338, 612 
Koester, D. \& Kepler, S. O. 2015, A\&A, 583, A86

Koester, D., Voss, B., Napiwotzki, R., Christlieb, N., Homeier, D., Lisker, T., Reimers, D., \& Heber, U. 2009, A\&A, 505, 441

Koester, D. \& Weidemann, V. 1982, A\&A, 108, 406

Lacombe, P. \& Fontaine, G. 1981, A\&AS, 43, 367

Lamb, D. Q. \& van Horn, H. M. 1975, ApJ, 200, 306

Landolt, A. U. 1992, AJ, 104, 340

-. 2009, AJ, 137, 4186

—. 2013, AJ, 146, 131

Landstreet, J. D., Bagnulo, S., Martin, A., \& Valyavin, G. 2016, A\&A, 591, A80

Landstreet, J. D., Bagnulo, S., Valyavin, G. G., Fossati, L., Jordan, S., Monin, D., \& Wade, G. A. 2012, A\&A, 545, A30

Lecavalier-Hurtubise, É. \& Bergeron, P. 2017, in Astronomical Society of the Pacific Conference Series, Vol. 509, 20th European White Dwarf Workshop, ed. P.-E. Tremblay, B. Gaensicke, \& T. Marsh, 169

Liebert, J., Bergeron, P., \& Holberg, J. B. 2005, ApJS, 156, 47

Limoges, M.-M., Bergeron, P., \& Lépine, S. 2015, ApJS, 219, 19

Maxted, P. F. L. \& Marsh, T. R. 1999, MNRAS, 307, 122

Maxted, P. F. L., Marsh, T. R., \& Moran, C. K. J. 2000, MNRAS, 319, 305

McCook, G. P. \& Sion, E. M. 1999, ApJS, 121, 1

Morales-Rueda, L., Marsh, T. R., Maxted, P. F. L., Nelemans, G., Karl, C., Napiwotzki, R., \& Moran, C. K. J. 2005, MNRAS, 359, 648

Napiwotzki, R., Christlieb, N., Drechsel, H., Hagen, H.-J., Heber, U., Homeier, D., Karl, C., Koester, D., Leibundgut, B., Marsh, T. R., Moehler, S., Nelemans, G., Pauli, E.-M., Reimers, D., Renzini, A., \& Yungelson, L. 2003, The Messenger, 112, 25

Nelemans, G., Napiwotzki, R., Karl, C., Marsh, T. R., Voss, B., Roelofs, G., Izzard, R. G., Montgomery, M., Reerink, T., Christlieb, N., \& Reimers, D. 2005, A\&A, 440, 1087 
Ouyed, R., Staff, J., \& Jaikumar, P. 2011, ApJ, 743, 116

Parsons, S. G., Gänsicke, B. T., Marsh, T. R., Ashley, R. P., Bours, M. C. P., Breedt, E., Burleigh, M. R., Copperwheat, C. M., Dhillon, V. S., Green, M., Hardy, L. K., Hermes, J. J., Irawati, P., Kerry, P., Littlefair, S. P., McAllister, M. J., Rattanasoon, S., Rebassa-Mansergas, A., Sahman, D. I., \& Schreiber, M. R. 2017, ArXiv e-prints

Press, W. H., Flannery, B. P., \& Teukolsky, S. A. 1986, Numerical recipes. The art of scientific computing, ed. Press, W. H., Flannery, B. P., \& Teukolsky, S. A.

Provencal, J. L., Shipman, H. L., Høg, E., \& Thejll, P. 1998, ApJ, 494, 759

Provencal, J. L., Shipman, H. L., Koester, D., Wesemael, F., \& Bergeron, P. 2002, ApJ, 568, 324

Reid, I. N. 1996, AJ, 111, 2000

Reindl, N., Rauch, T., Werner, K., Kepler, S. O., Gänsicke, B. T., \& Gentile Fusillo, N. P. 2014, A\&A, 572, A117

Ruiz, M. T. 1996, AJ, 111, 1267

Saffer, R. A., Liebert, J., \& Olszewski, E. W. 1988, ApJ, 334, 947

Sahu, K. C., Anderson, J., Casertano, S., Bond, H. E., Bergeron, P., Nelan, E. P., Pueyo, L., Brown, T. M., Bellini, A., Levay, Z. G., Sokol, J., aff1, Dominik, M., Calamida, A., Kains, N., \& Livio, M. 2017, Science, 356, 1046

Salaris, M., Domínguez, I., García-Berro, E., Hernanz, M., Isern, J., \& Mochkovitch, R. 1997, ApJ, 486, 413

Schmidt, H. 1996, A\&A, 311, 852

Subasavage, J. P., Henry, T. J., Bergeron, P., Dufour, P., \& Hambly, N. C. 2008, AJ, 136, 899

Subasavage, J. P., Henry, T. J., Bergeron, P., Dufour, P., Hambly, N. C., \& Beaulieu, T. D. 2007, AJ, 134, 252

Subasavage, J. P., Jao, W.-C., Henry, T. J., Bergeron, P., Dufour, P., Ianna, P. A., Costa, E., \& Méndez, R. A. 2009, AJ, 137, 4547 
Subasavage, J. P., Jao, W.-C., Henry, T. J., Harris, H. C., Dahn, C. C., Bergeron, P., Dufour, P., Dunlap, B. H., Barlow, B. N., Ianna, P. A., Lépine, S., \& Margheim, S. J. 2017, AJ, 154,32

Tremblay, P.-E. \& Bergeron, P. 2009, ApJ, 696, 1755

Tremblay, P.-E., Bergeron, P., \& Gianninas, A. 2011a, ApJ, 730, 128

Tremblay, P.-E., Bergeron, P., Kalirai, J. S., \& Gianninas, A. 2010, ApJ, 712, 1345

Tremblay, P.-E., Fontaine, G., Freytag, B., Steiner, O., Ludwig, H.-G., Steffen, M., Wedemeyer, S., \& Brassard, P. 2015, ApJ, 812, 19

Tremblay, P.-E., Gentile-Fusillo, N., Raddi, R., Jordan, S., Besson, C., Gänsicke, B. T., Parsons, S. G., Koester, D., Marsh, T., Bohlin, R., Kalirai, J., \& Deustua, S. 2017, MNRAS, 465, 2849

Tremblay, P.-E., Ludwig, H.-G., Steffen, M., Bergeron, P., \& Freytag, B. 2011b, A\&A, 531, L19

Tremblay, P.-E., Ludwig, H.-G., Steffen, M., \& Freytag, B. 2013, A\&A, 559, A104

Valyavin, G., Shulyak, D., Wade, G. A., Antonyuk, K., Zharikov, S. V., Galazutdinov, G. A., Plachinda, S., Bagnulo, S., Fox Machado, L., Alvarez, M., Clark, D. M., Lopez, J. M., Hiriart, D., Han, I., Jeon, Y.-B., Zurita, C., Mujica, R., Burlakova, T., Szeifert, T., \& Burenkov, A. 2014, Nature, 515, 88

van Altena, W. F., Lee, J. T., \& Hoffleit, E. D. 1995, The general catalogue of trigonometric [stellar] parallaxes

van Leeuwen, F. 2007, A\&A, 474, 653

Vennes, S., Thejll, P. A., Génova Galvan, R., \& Dupuis, J. 1997, ApJ, 480, 714

Voss, B., Koester, D., Napiwotzki, R., Christlieb, N., \& Reimers, D. 2007, A\&A, 470, 1079

Wegner, G. 1979, AJ, 84, 1384

-. 1983, AJ, 88, 109

Zuckerman, B., Koester, D., Melis, C., Hansen, B. M., \& Jura, M. 2007, ApJ, 671, 872

Zuckerman, B., Koester, D., Reid, I. N., \& Hünsch, M. 2003, ApJ, 596, 477 
Table 1. Adopted Physical Parameters of White Dwarfs with Reliable Data

\begin{tabular}{|c|c|c|c|c|c|c|c|c|c|c|}
\hline WD & Name & Spectral Type & $D_{\pi}(\mathrm{pc})$ & $T_{\text {eff }}(\mathrm{K})$ & Method $^{a}$ & $\log g$ & $R_{\pi} / R_{\odot}$ & $M / M_{\odot}$ & $\Delta D(\sigma)$ & Notes \\
\hline $0002+729$ & GD 408 & DBA & $34.7(5.8)$ & $14,406(235)$ & $\mathrm{S}$ & $8.26(0.10)$ & $0.0114(0.0019)$ & $0.87(0.36)$ & 0.39 & \\
\hline $0008+424$ & GD 5 & $\mathrm{DA}$ & $23.2(0.2)$ & 7121 (105) & $\mathrm{S}$ & $8.11(0.07)$ & $0.0125(0.0002)$ & $0.74(0.13)$ & 1.14 & \\
\hline $0011+000$ & G31-35 & DA & $29.7(4.3)$ & $9498(137)$ & $\mathrm{S}$ & $8.00(0.05)$ & $0.0096(0.0014)$ & $0.34(0.11)$ & 2.17 & \\
\hline $0030+444$ & G172-4 & $\mathrm{DA}$ & $71.6(4.1)$ & $10,277(151)$ & $\mathrm{S}$ & $8.05(0.05)$ & $0.0121(0.0007)$ & $0.60(0.10)$ & 0.36 & \\
\hline $0033+016$ & G1-7 & $\mathrm{DA}$ & $32.9(4.4)$ & $10,828(160)$ & $\mathrm{S}$ & $8.73(0.05)$ & $0.0079(0.0011)$ & $1.22(0.36)$ & 0.49 & \\
\hline $0034-602$ & LP $122-4$ & $\mathrm{DA}$ & $24.1(0.9)$ & $15,239(319)$ & $\mathrm{S}$ & $8.64(0.04)$ & $0.0084(0.0003)$ & $1.13(0.15)$ & 0.94 & \\
\hline $0053-117$ & L796-10 & DA & $22.8(0.2)$ & $7045(106)$ & $\mathrm{S}$ & $8.11(0.08)$ & $0.0128(0.0002)$ & $0.78(0.16)$ & 1.47 & \\
\hline $0101+048$ & G1-45 & DA & $20.9(1.7)$ & $8343(121)$ & $\mathrm{S}$ & $8.04(0.05)$ & $0.0158(0.0013)$ & $1.01(0.21)$ & 2.52 & 1 \\
\hline $0126+101$ & G2-40 & DA & $34.4(3.7)$ & $8548(123)$ & $\mathrm{S}$ & $7.60(0.05)$ & $0.0205(0.0022)$ & $0.61(0.15)$ & 1.81 & 1 \\
\hline $0133-116$ & Ross 548 & $\mathrm{DA}$ & $63.3(8.1)$ & $12,267(186)$ & $\mathrm{S}$ & $8.01(0.05)$ & $0.0249(0.0032)$ & $2.29(0.65)$ & 3.73 & \\
\hline $0135-052$ & L870-2 & $\mathrm{DA}$ & $12.0(0.5)$ & $7160(103)$ & $\mathrm{S}$ & $7.75(0.06)$ & $0.0201(0.0008)$ & $0.82(0.13)$ & 5.36 & 1 \\
\hline $0136+152$ & PG $0136+152$ & DA & $22.2(0.3)$ & $7972(114)$ & $\mathrm{S}$ & $8.20(0.05)$ & $0.0118(0.0002)$ & $0.80(0.10)$ & 1.46 & \\
\hline $0142+312$ & G72-31 & $\mathrm{DA}$ & $34.8(5.3)$ & $9223(134)$ & $\mathrm{S}$ & $8.13(0.05)$ & $0.0156(0.0024)$ & $1.21(0.41)$ & 1.61 & 1 \\
\hline $0148+467$ & GD 279 & $\mathrm{DA}$ & $15.5(0.8)$ & $13,944(275)$ & $\mathrm{S}$ & $8.05(0.04)$ & $0.0122(0.0007)$ & $0.61(0.09)$ & 0.35 & \\
\hline $0148+641$ & G244-36 & $\mathrm{DA}$ & $17.4(0.2)$ & $8879(127)$ & $\mathrm{S}$ & $8.20(0.05)$ & $0.0119(0.0002)$ & $0.82(0.09)$ & 1.79 & \\
\hline $0150+256$ & G94-21 & $\mathrm{DA}$ & $34.0(2.2)$ & $7677(153)$ & $\mathrm{P}$ & $7.94(0.13)$ & $0.0134(0.0010)$ & $0.57(0.19)$ & 0.09 & \\
\hline $0205+250$ & G35-29 & DA & $33.3(2.8)$ & $21,121(316)$ & $\mathrm{S}$ & $7.91(0.04)$ & $0.0133(0.0011)$ & $0.52(0.10)$ & 0.57 & \\
\hline $0208+396$ & G74-7 & DAZ & $16.7(1.0)$ & $7332(109)$ & $\mathrm{S}$ & $7.93(0.08)$ & $0.0125(0.0007)$ & $0.48(0.10)$ & 0.91 & \\
\hline $0213+396$ & GD 25 & DA & $19.7(0.3)$ & $9228(134)$ & $\mathrm{S}$ & $8.39(0.05)$ & $0.0098(0.0002)$ & $0.87(0.11)$ & 0.23 & \\
\hline $0220+222$ & G94-B5B & $\mathrm{DA}$ & $78.5(3.4)$ & $16,301(264)$ & $\mathrm{S}$ & $8.04(0.05)$ & $0.0121(0.0005)$ & $0.59(0.08)$ & 0.80 & \\
\hline $0226-329$ & MCT $0226-3255$ & $\mathrm{DA}$ & $45.9(2.7)$ & $22,770(361)$ & $\mathrm{S}$ & $7.96(0.05)$ & $0.0131(0.0008)$ & $0.57(0.09)$ & 0.46 & \\
\hline $0227+050$ & Feige 22 & $\mathrm{DA}$ & $26.7(3.7)$ & $19,514(302)$ & $\mathrm{S}$ & $7.95(0.05)$ & $0.0139(0.0020)$ & $0.63(0.19)$ & 0.18 & \\
\hline $0232+035$ & Feige 24 & $\mathrm{DA}+\mathrm{dM}$ & $76.6(6.3)$ & $67,133(1415)$ & $\mathrm{S}$ & $7.41(0.07)$ & $0.0205(0.0017)$ & $0.39(0.09)$ & 1.42 & \\
\hline $0243-026$ & LHS 1442 & DA & $20.9(0.2)$ & $6780(76)$ & $\mathrm{P}$ & $8.06(0.10)$ & $0.0112(0.0002)$ & $0.53(0.12)$ & 1.25 & \\
\hline $0250-007$ & LP 591-117 & $\mathrm{DA}$ & $47.6(2.0)$ & $8278(124)$ & $\mathrm{S}$ & $8.29(0.07)$ & $0.0122(0.0006)$ & $1.04(0.20)$ & 2.15 & \\
\hline $0255-705$ & LHS 1474 & $\mathrm{DA}$ & $24.1(1.3)$ & $10,743(167)$ & $\mathrm{S}$ & $8.07(0.06)$ & $0.0116(0.0007)$ & $0.58(0.10)$ & 0.76 & \\
\hline $0257+080$ & LHS 5064 & $\mathrm{DA}$ & $27.9(2.7)$ & $6619(130)$ & $\mathrm{P}$ & $7.78(0.14)$ & $0.0132(0.0014)$ & $0.39(0.15)$ & 0.73 & 2 \\
\hline $0310-688$ & LB 3303 & $\mathrm{DA}$ & $10.4(0.1)$ & $16,246(237)$ & $\mathrm{S}$ & $8.14(0.04)$ & $0.0120(0.0002)$ & $0.72(0.08)$ & 0.45 & \\
\hline $0311-649$ & LEHPM 1-3159 & $\mathrm{DA}$ & $32.5(1.3)$ & $13,135(290)$ & $\mathrm{S}$ & $7.83(0.06)$ & $0.0187(0.0008)$ & $0.86(0.14)$ & 4.53 & 1 \\
\hline $0326-273$ & L587-55A & DA & $24.2(0.8)$ & $9207(133)$ & $\mathrm{S}$ & $7.71(0.06)$ & $0.0188(0.0006)$ & $0.65(0.09)$ & 4.09 & 1 \\
\hline $0401+250$ & G8-8 & $\mathrm{DA}$ & $26.8(2.0)$ & $12,482(191)$ & $\mathrm{S}$ & $8.10(0.05)$ & $0.0125(0.0010)$ & $0.71(0.14)$ & 0.43 & \\
\hline $0413-077$ & 40 Eri B & $\mathrm{DA}$ & $4.98(0.06)$ & $17,099(256)$ & $\mathrm{S}$ & $7.95(0.04)$ & $0.0131(0.0002)$ & $0.57(0.06)$ & 0.63 & \\
\hline $0415-594$ & HD $27442 B$ & $\mathrm{DA}$ & $18.2(0.2)$ & $15,424(270)$ & $\mathrm{S}$ & $7.98(0.05)$ & $0.0132(0.0010)$ & $0.60(0.12)$ & 0.03 & \\
\hline $0419-487$ & LHS 1660 & $\mathrm{DA}+\mathrm{dM}$ & $20.1(0.5)$ & $6354(261)$ & $\mathrm{P}$ & $7.38(0.18)$ & $0.0204(0.0022)$ & $0.36(0.18)$ & 0.70 & \\
\hline $0453+418$ & GD 64 & $\mathrm{DA}$ & $43.9(5.1)$ & $14,523(248)$ & $\mathrm{S}$ & $7.85(0.05)$ & $0.0166(0.0019)$ & $0.71(0.18)$ & 1.15 & \\
\hline
\end{tabular}


Table 1-Continued

\begin{tabular}{|c|c|c|c|c|c|c|c|c|c|c|}
\hline WD & Name & Spectral Type & $D_{\pi}(\mathrm{pc})$ & $T_{\text {eff }}(\mathrm{K})$ & Method $^{a}$ & $\log g$ & $R_{\pi} / R_{\odot}$ & $M / M_{\odot}$ & $\Delta D(\sigma)$ & Notes \\
\hline $0457-004$ & G84-26 & DA & $24.9(0.1)$ & $11,121(166)$ & $\mathrm{S}$ & $8.81(0.05)$ & $0.0069(0.0001)$ & $1.14(0.13)$ & 0.30 & \\
\hline $0501+527$ & G191-B2B & DA & $59.9(11.0)$ & $60,701(988)$ & S & $7.53(0.05)$ & $0.0228(0.0042)$ & $0.65(0.26)$ & 0.48 & \\
\hline $0518+333$ & G86-B1B & $\mathrm{DA}+\mathrm{dM}$ & $65.4(9.6)$ & $8963(129)$ & S & $8.06(0.05)$ & $0.0161(0.0024)$ & $1.08(0.35)$ & 1.58 & \\
\hline $0612+177$ & G104-27 & DA & $42.0(5.6)$ & $26,097(380)$ & $\mathrm{S}$ & $7.99(0.04)$ & $0.0133(0.0018)$ & $0.62(0.18)$ & 0.05 & \\
\hline $0615-591$ & L182-61 & DB & $36.4(0.7)$ & $15,746(238)$ & $\mathrm{S}$ & $8.04(0.06)$ & $0.0130(0.0003)$ & $0.67(0.10)$ & 1.00 & \\
\hline $0628-020$ & LP $600-42$ & $\mathrm{DA}+\mathrm{dM}$ & $21.5(0.8)$ & $6741(380)$ & $\mathrm{P}$ & $7.92(0.12)$ & $0.0129(0.0013)$ & $0.50(0.17)$ & 0.33 & \\
\hline $0642-166$ & Sirius B & $\mathrm{DA}$ & $2.64(0.01)$ & $26,083(378)$ & S & $8.61(0.04)$ & $0.0079(0.0002)$ & $0.94(0.11)$ & 0.72 & \\
\hline $0642-285$ & LP 895-41 & DA & $65.2(2.3)$ & $9230(133)$ & S & $7.95(0.05)$ & $0.0131(0.0011)$ & $0.56(0.11)$ & 0.13 & \\
\hline $0644+025$ & G108-26 & DA & $18.2(0.1)$ & 7085 (106) & $\mathrm{S}$ & $8.52(0.07)$ & $0.0083(0.0001)$ & $0.85(0.15)$ & 0.76 & \\
\hline $0644+375$ & G87-7 & DA & $15.7(0.9)$ & $21,996(254)$ & $\mathrm{S}$ & $8.15(0.04)$ & $0.0106(0.0006)$ & $0.58(0.09)$ & 1.67 & 3 \\
\hline $0659-063$ & LHS 1892 & DA & $20.6(0.1)$ & $6506(148)$ & $\mathrm{P}$ & $8.03(0.11)$ & $0.0124(0.0004)$ & $0.61(0.16)$ & 0.04 & \\
\hline $0752+365$ & G90-28 & DA & $33.6(4.3)$ & 7712 (111) & S & $7.98(0.06)$ & $0.0111(0.0014)$ & $0.43(0.13)$ & 1.21 & \\
\hline $0816+387$ & G111-71 & DA & $39.7(4.0)$ & $7544(123)$ & $\mathrm{P}$ & $8.10(0.09)$ & $0.0112(0.0012)$ & $0.57(0.17)$ & 0.58 & \\
\hline $0827+328$ & LHS 2022 & DA & $22.0(1.9)$ & $7268(106)$ & $\mathrm{S}$ & $8.37(0.06)$ & $0.0095(0.0008)$ & $0.78(0.18)$ & 0.31 & \\
\hline $0839-327$ & LHS 253 & DA & $8.48(0.08)$ & $9093(130)$ & $\mathrm{S}$ & $7.76(0.05)$ & $0.0146(0.0002)$ & $0.45(0.05)$ & 0.63 & \\
\hline $0842+490$ & HD 74389B & DA & $111.5(7.1)$ & $41,885(731)$ & S & $7.92(0.07)$ & $0.0114(0.0007)$ & $0.39(0.08)$ & 2.93 & \\
\hline $0913+442$ & G116-16 & DA & $28.4(3.3)$ & $8614(125)$ & S & $8.07(0.05)$ & $0.0109(0.0013)$ & $0.51(0.14)$ & 0.97 & \\
\hline $0928-713$ & BPM 5639 & DA & $26.0(0.4)$ & $8370(120)$ & S & $8.16(0.05)$ & $0.0114(0.0002)$ & $0.69(0.08)$ & 0.13 & \\
\hline $0930+294$ & G117-25 & DA & $31.4(4.6)$ & $8250(120)$ & S & $8.46(0.06)$ & $0.0097(0.0014)$ & $1.00(0.33)$ & 0.36 & \\
\hline $0942+236 \mathrm{~A}$ & LP 370-50 & DA & $46.5(9.2)$ & 7125 (109) & $\mathrm{S}$ & $7.80(0.10)$ & $0.0098(0.0019)$ & $0.22(0.10)$ & 2.16 & \\
\hline $0942+236 B$ & LP $370-51$ & DA & $46.5(9.2)$ & 7019 (133) & $\mathrm{P}$ & $8.17(0.13)$ & $0.0094(0.0019)$ & $0.47(0.25)$ & 0.94 & \\
\hline $0943+441$ & G116-52 & DA & $33.2(3.3)$ & $13,771(222)$ & $\mathrm{S}$ & $7.59(0.05)$ & $0.0181(0.0018)$ & $0.46(0.11)$ & 0.60 & \\
\hline $0955+247$ & G49-33 & DA & $24.1(2.6)$ & $8529(123)$ & $\mathrm{S}$ & $8.18(0.05)$ & $0.0107(0.0012)$ & $0.63(0.16)$ & 0.52 & \\
\hline $1012+083 \mathrm{~A}$ & G43-38 & $\mathrm{DA}$ & $28.5(3.3)$ & $6689(81)$ & $\mathrm{P}$ & $7.89(0.12)$ & $0.0125(0.0015)$ & $0.44(0.17)$ & 0.65 & \\
\hline $1016-308$ & LP 904-3 & $\mathrm{DA}$ & $50.9(3.2)$ & $16,297(243)$ & S & $8.18(0.04)$ & $0.0129(0.0008)$ & $0.92(0.15)$ & 1.60 & \\
\hline $1019+637$ & LP $62-147$ & DA & $16.2(1.0)$ & $6777(75)$ & $\mathrm{P}$ & $7.90(0.08)$ & $0.0126(0.0008)$ & $0.47(0.11)$ & 0.85 & \\
\hline $1104+602$ & G197-4 & DA & $43.9(8.8)$ & $18,294(273)$ & S & $8.11(0.04)$ & $0.0151(0.0030)$ & $1.07(0.46)$ & 1.01 & \\
\hline $1105-340$ & SCR J1107-342 & DA & $25.6(0.4)$ & $13,891(271)$ & S & $8.06(0.04)$ & $0.0117(0.0002)$ & $0.57(0.06)$ & 1.64 & 3 \\
\hline $1121+216$ & Ross 627 & $\mathrm{DA}$ & $13.6(0.6)$ & $7346(107)$ & $\mathrm{S}$ & $8.10(0.06)$ & $0.0115(0.0005)$ & $0.61(0.11)$ & 0.57 & \\
\hline $1124-293$ & ESO 439-80 & $\mathrm{DA}$ & $32.3(1.6)$ & $9348(134)$ & $\mathrm{S}$ & $7.98(0.05)$ & $0.0125(0.0006)$ & $0.54(0.08)$ & 0.67 & \\
\hline $1130+189$ & LP 433-6 & DA & $216.0(34.9)$ & $10,886(174)$ & $\mathrm{S}$ & $8.40(0.06)$ & $0.0206(0.0033)$ & $3.85(1.39)$ & 3.24 & 1 \\
\hline $1134+300$ & GD 140 & DA & $15.8(0.9)$ & $22,313(339)$ & S & $8.56(0.05)$ & $0.0086(0.0005)$ & $0.98(0.15)$ & 0.04 & \\
\hline $1143+321$ & G148-7 & DA & $30.2(3.0)$ & $16,332(245)$ & $\mathrm{S}$ & $8.17(0.04)$ & $0.0121(0.0012)$ & $0.79(0.18)$ & 0.46 & \\
\hline $1147+255$ & LP $375-51$ & DA & $47.4(8.8)$ & $10,072(148)$ & $\mathrm{S}$ & $8.00(0.05)$ & $0.0121(0.0023)$ & $0.54(0.22)$ & 0.29 & \\
\hline $1202-232$ & LP 852-7 & DA & $10.9(0.1)$ & $8608(124)$ & S & $7.99(0.05)$ & $0.0135(0.0002)$ & $0.64(0.08)$ & 1.29 & \\
\hline
\end{tabular}


Table 1-Continued

\begin{tabular}{|c|c|c|c|c|c|c|c|c|c|c|}
\hline WD & Name & Spectral Type & $D_{\pi}(\mathrm{pc})$ & $T_{\text {eff }}(\mathrm{K})$ & Method $^{a}$ & $\log g$ & $R_{\pi} / R_{\odot}$ & $M / M_{\odot}$ & $\Delta D(\sigma)$ & Notes \\
\hline $1214+032$ & LP 554-63 & DA & $21.6(1.6)$ & $6751(151)$ & $\mathrm{P}$ & $7.81(0.14)$ & $0.0128(0.0011)$ & $0.39(0.14)$ & 0.93 & \\
\hline $1223-659$ & L104-2 & DA & $16.3(0.3)$ & 7491 (107) & S & $7.76(0.05)$ & $0.0147(0.0003)$ & $0.45(0.06)$ & 0.35 & \\
\hline $1236-495$ & BPM 37093 & DA & $15.3(0.2)$ & $11,620(189)$ & S & $8.72(0.05)$ & $0.0077(0.0001)$ & $1.13(0.14)$ & 0.71 & \\
\hline $1242-105$ & LP 736-4 & DA & $39.7(1.2)$ & 8145 (119) & S & $7.85(0.06)$ & $0.0225(0.0007)$ & $1.31(0.21)$ & 9.42 & 1 \\
\hline $1257+278$ & G149-28 & DAZ & $33.6(4.9)$ & $8612(126)$ & S & $8.04(0.06)$ & $0.0125(0.0018)$ & $0.63(0.21)$ & 0.03 & \\
\hline $1304+227$ & LP 378-537 & $\mathrm{DA}$ & $77.2(3.5)$ & $11,119(173)$ & S & $8.21(0.05)$ & $0.0118(0.0005)$ & $0.83(0.13)$ & 0.97 & \\
\hline $1314+293$ & HZ 43A & $\mathrm{DA}+\mathrm{dM}$ & $58.0(2.6)$ & $55,396(1170)$ & S & $8.01(0.07)$ & $0.0137(0.0007)$ & $0.71(0.14)$ & 0.01 & \\
\hline $1314-153$ & LHS 2712 & DA & $58.1(4.3)$ & $15,736(261)$ & S & $7.90(0.05)$ & $0.0142(0.0011)$ & $0.58(0.11)$ & 0.25 & \\
\hline $1325+581$ & G199-71 & DA & $36.1(7.5)$ & $6725(83)$ & $\mathrm{P}$ & $7.99(0.21)$ & $0.0114(0.0024)$ & $0.46(0.30)$ & 0.48 & \\
\hline $1327-083$ & Wolf $485 \mathrm{~A}$ & DA & $16.1(0.3)$ & $14,714(237)$ & $\mathrm{S}$ & $8.00(0.05)$ & $0.0131(0.0003)$ & $0.62(0.07)$ & 0.20 & \\
\hline $1333+487$ & GD 325 & DBA & $35.0(4.0)$ & $15,420(242)$ & $\mathrm{S}$ & $8.01(0.09)$ & $0.0120(0.0014)$ & $0.53(0.17)$ & 0.42 & \\
\hline $1337+705$ & G238-44 & $\mathrm{DA}$ & $26.1(2.1)$ & $21,311(328)$ & $\mathrm{S}$ & $7.96(0.05)$ & $0.0129(0.0010)$ & $0.56(0.11)$ & 0.46 & \\
\hline $1344+106$ & LHS 2800 & DA & $19.7(1.4)$ & $6968(107)$ & S & $8.00(0.08)$ & $0.0120(0.0009)$ & $0.52(0.13)$ & 0.68 & \\
\hline $1354+340$ & G165-B5B & DA & $92.7(5.0)$ & $14,608(315)$ & S & $7.97(0.06)$ & $0.0129(0.0007)$ & $0.57(0.10)$ & 0.38 & \\
\hline $1408+323$ & GD 163 & DA & $37.7(4.0)$ & $19,015(287)$ & S & $8.00(0.04)$ & $0.0117(0.0013)$ & $0.50(0.12)$ & 0.99 & \\
\hline $1418-088$ & G124-26 & DA & $39.2(6.1)$ & 8061 (117) & S & $8.10(0.06)$ & $0.0168(0.0026)$ & $1.29(0.45)$ & 1.81 & 1 \\
\hline $1422+095$ & GD 165 & DA & $31.5(2.5)$ & $12,227(186)$ & S & $8.08(0.05)$ & $0.0118(0.0009)$ & $0.61(0.12)$ & 0.43 & \\
\hline $1447-190$ & LP 801-14 & DA & $47.4(1.9)$ & $7132(229)$ & $\mathrm{P}$ & $7.80(0.20)$ & $0.0204(0.0013)$ & $0.96(0.47)$ & 2.95 & 1 \\
\hline $1455+298$ & LHS 3007 & DA & $33.6(4.7)$ & $7290(105)$ & $\mathrm{S}$ & $7.90(0.06)$ & $0.0153(0.0021)$ & $0.68(0.22)$ & 0.79 & \\
\hline $1509+322$ & GD 178 & DA & $45.7(9.1)$ & $14,807(259)$ & S & $8.15(0.05)$ & $0.0161(0.0032)$ & $1.34(0.57)$ & 1.38 & \\
\hline $1544-377$ & L481-60 & DA & $15.3(0.2)$ & $10,402(150)$ & S & $7.97(0.05)$ & $0.0134(0.0003)$ & $0.62(0.07)$ & 0.77 & \\
\hline $1554+215$ & PG $1554+215$ & DA & $102.8(7.2)$ & $27,123(410)$ & $\mathrm{S}$ & $7.87(0.05)$ & $0.0136(0.0010)$ & $0.50(0.09)$ & 0.90 & \\
\hline $1559+369$ & Ross 808 & DA & $33.1(3.7)$ & $11,187(165)$ & $\mathrm{S}$ & $7.99(0.05)$ & $0.0131(0.0014)$ & $0.61(0.15)$ & 0.10 & \\
\hline $1606+422$ & Case 2 & DA & $45.0(7.3)$ & $13,064(216)$ & $\mathrm{S}$ & $7.84(0.05)$ & $0.0202(0.0033)$ & $1.03(0.36)$ & 1.78 & 1 \\
\hline $1609+135$ & LHS 3163 & DA & $17.3(1.4)$ & 9349 (136) & S & $8.60(0.05)$ & $0.0066(0.0005)$ & $0.63(0.13)$ & 2.49 & \\
\hline $1619+123$ & PG $1619+123$ & DA & $56.5(1.7)$ & $17,156(259)$ & $\mathrm{S}$ & $7.88(0.05)$ & $0.0141(0.0004)$ & $0.54(0.07)$ & 0.13 & \\
\hline $1620-391$ & $\mathrm{CD}-3810980$ & DA & $12.8(0.1)$ & $25,954(368)$ & $\mathrm{S}$ & $7.96(0.04)$ & $0.0120(0.0001)$ & $0.49(0.05)$ & 3.44 & 3 \\
\hline $1625+093$ & G138-31 & $\mathrm{DA}$ & $23.4(2.0)$ & $7129(134)$ & $\mathrm{S}$ & $8.60(0.16)$ & $0.0087(0.0008)$ & $1.10(0.45)$ & 0.37 & \\
\hline $1633+433$ & G180-63 & DAZ & $15.1(0.7)$ & $6625(61)$ & $\mathrm{P}$ & $7.92(0.15)$ & $0.0116(0.0006)$ & $0.41(0.14)$ & 1.33 & \\
\hline $1635+137$ & G138-47 & $\mathrm{DA}$ & $39.2(7.3)$ & $6811(92)$ & $\mathrm{P}$ & $7.76(0.13)$ & $0.0109(0.0020)$ & $0.25(0.12)$ & 1.66 & \\
\hline $1637+335$ & G180-65 & DA & $29.2(3.3)$ & $10,151(147)$ & $\mathrm{S}$ & $8.05(0.05)$ & $0.0119(0.0013)$ & $0.57(0.15)$ & 0.39 & \\
\hline $1639+153$ & LHS 3236 & DA & $30.9(0.2)$ & $7452(110)$ & $\mathrm{S}$ & $8.49(0.07)$ & $0.0128(0.0001)$ & $1.84(0.29)$ & 7.69 & 1 \\
\hline $1645+325$ & GD 358 & DB & $36.6(4.5)$ & $24,937(1018)$ & $\mathrm{S}$ & $7.92(0.05)$ & $0.0110(0.0014)$ & $0.37(0.10)$ & 1.85 & \\
\hline $1647+591$ & G226-29 & DA & $11.0(0.1)$ & $12,517(195)$ & $\mathrm{S}$ & $8.34(0.05)$ & $0.0103(0.0001)$ & $0.83(0.09)$ & 0.30 & \\
\hline $1655+215$ & LHS 3254 & DA & $23.0(1.6)$ & $9229(133)$ & $\mathrm{S}$ & $8.07(0.05)$ & $0.0137(0.0010)$ & $0.81(0.15)$ & 1.42 & \\
\hline
\end{tabular}


Table 1-Continued

\begin{tabular}{|c|c|c|c|c|c|c|c|c|c|c|}
\hline WD & Name & Spectral Type & $D_{\pi}(\mathrm{pc})$ & $T_{\text {eff }}(\mathrm{K})$ & Method $^{a}$ & $\log g$ & $R_{\pi} / R_{\odot}$ & $M / M_{\odot}$ & $\Delta D(\sigma)$ & Notes \\
\hline $1659-531$ & L268-92 & DA & $27.2(0.5)$ & $15,507(230)$ & S & $8.07(0.04)$ & $0.0124(0.0003)$ & $0.66(0.07)$ & 0.14 & \\
\hline $1706+332$ & G181-B5B & DA & $71.5(2.7)$ & $12,774(211)$ & S & $8.06(0.05)$ & $0.0124(0.0005)$ & $0.64(0.09)$ & 0.07 & \\
\hline $1713+695$ & G240-51 & DA & $25.0(2.8)$ & $15,952(243)$ & S & $8.00(0.05)$ & $0.0122(0.0014)$ & $0.55(0.14)$ & 0.53 & \\
\hline $1716+020$ & G19-20 & DA & $40.8(7.2)$ & $13,255(268)$ & S & $7.88(0.05)$ & $0.0143(0.0025)$ & $0.56(0.22)$ & 0.12 & \\
\hline $1733-544$ & L270-137 & DA & $21.9(0.4)$ & $6542(85)$ & $\mathrm{P}$ & $8.35(0.11)$ & $0.0115(0.0003)$ & $1.08(0.29)$ & 1.69 & \\
\hline $1736+052$ & G140-2 & DA & $42.2(7.1)$ & $8969(132)$ & S & $8.14(0.06)$ & $0.0116(0.0020)$ & $0.68(0.26)$ & 0.02 & \\
\hline $1743-132$ & G154-B5B & DA & $38.5(1.1)$ & $12,850(210)$ & S & $8.00(0.05)$ & $0.0143(0.0005)$ & $0.74(0.10)$ & 2.13 & \\
\hline $1756+827$ & LHS 56 & DA & $16.9(1.4)$ & $7227(106)$ & S & $7.89(0.07)$ & $0.0139(0.0011)$ & $0.55(0.13)$ & 0.20 & \\
\hline $1824+040$ & Ross 137 & $\mathrm{DA}$ & $54.9(7.1)$ & $12,252(190)$ & $\mathrm{S}$ & $7.70(0.05)$ & $0.0252(0.0032)$ & $1.15(0.33)$ & 2.90 & 1 \\
\hline $1826-045$ & G21-16 & DA & $28.7(3.2)$ & $9125(131)$ & $\mathrm{S}$ & $8.00(0.05)$ & $0.0140(0.0015)$ & $0.70(0.18)$ & 0.70 & \\
\hline $1840+042$ & GD 215 & DA & $24.8(2.1)$ & $8877(128)$ & S & $8.19(0.05)$ & $0.0117(0.0010)$ & $0.77(0.16)$ & 0.39 & \\
\hline $1855+338$ & G207-9 & DA & $32.8(4.8)$ & $12,137(184)$ & S & $8.33(0.05)$ & $0.0106(0.0016)$ & $0.88(0.28)$ & 0.26 & \\
\hline $1916-362$ & SCR J1920-361 & DB & $37.4(1.6)$ & $25,383(3225)$ & S & $7.86(0.10)$ & $0.0113(0.0005)$ & $0.34(0.08)$ & 2.62 & \\
\hline $1919+145$ & GD 219 & DA & $19.8(2.2)$ & $15,080(258)$ & $\mathrm{S}$ & $8.20(0.05)$ & $0.0111(0.0012)$ & $0.71(0.18)$ & 0.15 & \\
\hline $1935+276$ & G185-32 & DA & $17.9(0.9)$ & $12,381(186)$ & S & $8.09(0.05)$ & $0.0120(0.0006)$ & $0.64(0.10)$ & 0.21 & \\
\hline $1936+327$ & GD 222 & DA & $34.8(2.9)$ & $22,228(339)$ & S & $7.91(0.05)$ & $0.0116(0.0010)$ & $0.40(0.08)$ & 2.22 & \\
\hline $1940+374$ & L1573-31 & DB & $49.3(6.9)$ & $16,851(267)$ & S & $8.07(0.10)$ & $0.0125(0.0018)$ & $0.68(0.25)$ & 0.19 & \\
\hline $1943+163$ & G142-50 & $\mathrm{DA}$ & $41.5(4.7)$ & $20,372(310)$ & S & $7.94(0.05)$ & $0.0119(0.0014)$ & $0.45(0.11)$ & 1.24 & \\
\hline $1950+250$ & GD 385 & $\mathrm{DA}$ & $38.0(4.7)$ & $11,897(181)$ & S & $8.05(0.05)$ & $0.0101(0.0012)$ & $0.41(0.11)$ & 1.82 & \\
\hline $1953-011$ & LHS 3501 & DA & $11.9(0.5)$ & $7765(113)$ & $\mathrm{S}$ & $8.20(0.06)$ & $0.0117(0.0005)$ & $0.79(0.13)$ & 0.75 & 2 \\
\hline $2007-219$ & L710-30 & DA & $26.2(0.6)$ & $9842(144)$ & S & $8.01(0.05)$ & $0.0125(0.0003)$ & $0.58(0.08)$ & 0.50 & \\
\hline $2007-303$ & L565-18 & DA & $16.4(1.2)$ & $16,041(233)$ & $\mathrm{S}$ & $7.95(0.04)$ & $0.0130(0.0010)$ & $0.55(0.10)$ & 0.41 & \\
\hline $2032+248$ & Wolf 1346 & DA & $15.5(0.6)$ & $20,851(303)$ & S & $7.97(0.04)$ & $0.0139(0.0006)$ & $0.66(0.09)$ & 0.74 & \\
\hline $2035-369$ & L495-42 & DA & $31.9(1.0)$ & $9647(139)$ & S & $8.16(0.05)$ & $0.0123(0.0004)$ & $0.80(0.10)$ & 1.45 & \\
\hline $2039-202$ & L711-10 & $\mathrm{DA}$ & $20.7(1.6)$ & $20,031(295)$ & S & $7.96(0.04)$ & $0.0130(0.0010)$ & $0.57(0.11)$ & 0.38 & \\
\hline $2040-392$ & L495-82 & DA & $22.6(0.5)$ & $11,029(160)$ & S & $8.08(0.05)$ & $0.0122(0.0003)$ & $0.65(0.08)$ & 0.05 & \\
\hline $2047+372$ & G210-36 & DA & $17.1(0.1)$ & $14,628(279)$ & $\mathrm{S}$ & $8.33(0.04)$ & $0.0103(0.0001)$ & $0.83(0.09)$ & 0.25 & 2 \\
\hline $2048+809$ & LP $25-436$ & DA & $85.7(7.6)$ & $8365(124)$ & $\mathrm{S}$ & $8.23(0.07)$ & $0.0195(0.0018)$ & $2.39(0.58)$ & 4.77 & 1 \\
\hline $2105-820$ & L24-52 & DA & $15.4(0.3)$ & $9797(141)$ & S & $8.27(0.05)$ & $0.0108(0.0002)$ & $0.78(0.10)$ & 0.20 & $\begin{array}{l}1 \\
2\end{array}$ \\
\hline $2109+011$ & PM J21117+0120 & $\mathrm{DA}$ & $61.1(3.7)$ & $16,506(256)$ & S & $8.07(0.05)$ & $0.0124(0.0009)$ & $0.66(0.11)$ & 0.04 & \\
\hline $2111+261$ & G187-32 & DA & $31.5(3.7)$ & $8525(124)$ & S & $8.15(0.06)$ & $0.0169(0.0020)$ & $1.48(0.41)$ & 2.60 & 1 \\
\hline $2117+539$ & G231-40 & DA & $17.3(0.3)$ & $14,648(237)$ & $\mathrm{S}$ & $7.91(0.05)$ & $0.0137(0.0003)$ & $0.56(0.06)$ & 0.10 & \\
\hline $2124+550$ & Ross 198 & DA & $33.2(5.0)$ & $14,620(383)$ & S & $8.48(0.05)$ & $0.0088(0.0013)$ & $0.85(0.28)$ & 0.23 & \\
\hline $2126+734$ & G261-43 & DA & $21.2(1.1)$ & $16,040(236)$ & S & $7.99(0.04)$ & $0.0128(0.0007)$ & $0.58(0.08)$ & 0.43 & \\
\hline $2129+000$ & G26-10 & DB & $43.2(1.0)$ & $14,383(231)$ & S & $8.27(0.12)$ & $0.0120(0.0003)$ & $0.97(0.28)$ & 1.49 & \\
\hline
\end{tabular}


Table 1-Continued

\begin{tabular}{|c|c|c|c|c|c|c|c|c|c|c|}
\hline WD & Name & Spectral Type & $D_{\pi}(\mathrm{pc})$ & $T_{\text {eff }}(\mathrm{K})$ & Method $^{a}$ & $\log g$ & $R_{\pi} / R_{\odot}$ & $M / M_{\odot}$ & $\Delta D(\sigma)$ & Notes \\
\hline $2133-135$ & Ross 203 & DA & $24.7(0.8)$ & $10,193(149)$ & $\mathrm{S}$ & $7.86(0.05)$ & $0.0154(0.0005)$ & $0.63(0.09)$ & 1.95 & \\
\hline $2136+229$ & G126-18 & DA & $41.7(5.5)$ & $10,127(148)$ & $\mathrm{S}$ & $7.93(0.05)$ & $0.0138(0.0018)$ & $0.59(0.17)$ & 0.19 & \\
\hline $2136+828$ & G261-45 & DA & $26.0(3.1)$ & $17,698(263)$ & S & $7.97(0.04)$ & $0.0134(0.0016)$ & $0.61(0.16)$ & 0.04 & \\
\hline $2149+021$ & G93-48 & DA & $24.4(1.5)$ & $18,202(265)$ & S & $8.01(0.04)$ & $0.0138(0.0008)$ & $0.71(0.11)$ & 0.89 & \\
\hline $2159-754$ & LHS 3752 & DA & $19.9(0.5)$ & $8748(129)$ & $\mathrm{S}$ & $8.66(0.06)$ & $0.0086(0.0002)$ & $1.22(0.18)$ & 1.61 & \\
\hline $2207+142$ & G18-34 & DA & $25.1(2.8)$ & 7567 (110) & $\mathrm{S}$ & $8.19(0.06)$ & $0.0108(0.0012)$ & $0.66(0.17)$ & 0.35 & \\
\hline $2229+235$ & HS $2229+2335$ & $\mathrm{DA}$ & $110.9(10.5)$ & $19,952(360)$ & $\mathrm{S}$ & $8.05(0.06)$ & $0.0128(0.0012)$ & $0.67(0.16)$ & 0.13 & \\
\hline $2246+223$ & G67-23 & DA & $18.9(1.5)$ & $10,484(153)$ & $\mathrm{S}$ & $8.65(0.05)$ & $0.0083(0.0006)$ & $1.11(0.21)$ & 0.52 & \\
\hline $2253-081$ & G156-64 & DA & $35.8(0.7)$ & $6678(128)$ & $\mathrm{P}$ & $7.97(0.18)$ & $0.0127(0.0004)$ & $0.56(0.24)$ & 0.14 & \\
\hline $2258+406$ & G216-B14B & DA & $71.6(3.8)$ & $9852(146)$ & $\mathrm{S}$ & $8.18(0.06)$ & $0.0121(0.0012)$ & $0.81(0.19)$ & 0.66 & \\
\hline $2326+049$ & G29-38 & DA & $17.5(0.1)$ & $11,315(180)$ & $\mathrm{S}$ & $8.02(0.06)$ & $0.0127(0.0001)$ & $0.62(0.08)$ & 0.01 & \\
\hline $2336-079$ & GD 1212 & DA & $15.9(0.4)$ & $11,037(162)$ & $\mathrm{S}$ & $8.03(0.05)$ & $0.0107(0.0003)$ & $0.45(0.06)$ & 3.76 & \\
\hline $2341+322$ & G130-5 & DA & $18.8(0.1)$ & $13,076(218)$ & $\mathrm{S}$ & $8.00(0.05)$ & $0.0127(0.0001)$ & $0.59(0.07)$ & 0.41 & \\
\hline $2349-031$ & LHS 4033 & DA & $29.5(0.5)$ & $10,528(162)$ & $\mathrm{S}$ & $9.34(0.06)$ & $0.0039(0.0001)$ & $1.19(0.16)$ & 0.76 & \\
\hline $2350-083$ & G273-B1B & DA & $100.4(11.5)$ & $18,832(353)$ & $\mathrm{S}$ & $7.98(0.06)$ & $0.0115(0.0013)$ & $0.46(0.13)$ & 1.18 & \\
\hline $2351-335$ & LHS 4040 & DA & $23.4(1.3)$ & $8663(125)$ & $\mathrm{S}$ & $8.03(0.06)$ & $0.0131(0.0008)$ & $0.67(0.12)$ & 0.66 & \\
\hline $2351-368$ & LHS 4041 & DA & $74.6(8.5)$ & $13,875(351)$ & $\mathrm{S}$ & $8.04(0.05)$ & $0.0154(0.0018)$ & $0.95(0.24)$ & 1.54 & \\
\hline $2359-434$ & LHS 1005 & DA & $8.23(0.06)$ & $8506(122)$ & $\mathrm{S}$ & $8.38(0.05)$ & $0.0097(0.0001)$ & $0.82(0.09)$ & 0.26 & 2 \\
\hline
\end{tabular}

Note. - ${ }^{a}$ S: spectroscopic effective temperature; P: photometric effective temperature. (1) Unresolved double degenerate system. The parameters derived under the assumption of a single star are meaningless. Atmospheric parameters of both components are given in Figure 16. (2) Weakly magnetic white dwarf. (3) Possible iron-core white dwarf. 